\title{
Spectroscopic Measurements of Path-Averaged Species Correlations in Turbulent Flare Plumes
}

by

Scott P. Seymour

A thesis submitted to The Faculty of Graduate Studies and Research

in partial fulfilment of the degree requirements of

Master of Applied Science in Mechanical Engineering

Ottawa-Carleton Institute for

Mechanical and Aerospace Engineering

Department of Mechanical and Aerospace Engineering

Carleton University

Ottawa, Ontario, Canada

May 2019

Copyright (C) 2019 - Scott P. Seymour 
The undersigned recommend to

the Faculty of Graduate Studies and Research

acceptance of the thesis

\title{
Spectroscopic Measurements of Path-Averaged Species \\ Correlations in Turbulent Flare Plumes
}

\author{
Submitted by Scott P. Seymour \\ in partial fulfilment of the requirements for the degree of \\ Master of Applied Science in Mechanical Engineering
}

Dr. Matthew Johnson, Thesis Supervisor

Dr. Ron Miller, Chair, Department of Mechanical and Aerospace Engineering

Carleton University

2019 


\section{Abstract}

Flaring is the common upstream oil and gas industry practice of disposing unwanted combustible gas in a turbulent diffusion flame open to atmosphere. Pollutant emissions from flaring remain uncertain and most measurement techniques rely on the assumption that combustion-derived species are well-mixed and therefore well-correlated in the flare plume. This thesis presents a spectroscopic measurement technique used to measure path-averaged species correlation in turbulent flare plumes to assess this assumption. Tunable diode laser absorption spectroscopy (TDLAS) and line-of-sight attenuation (LOSA) techniques are used to measure $\mathrm{H}_{2} \mathrm{O}$ and soot, respectively. The spectroscopic techniques were first validated using synthetic data generated from a large eddy simulation of a turbulent flare plume. An experimental apparatus was subsequently developed and used to measure species correlation in lab-scale turbulent flare plumes. Results suggest that the instantaneous ratio of path-averaged $\mathrm{H}_{2} \mathrm{O}$ and soot in the plume follows a skewed distribution, such that flare emission measurements based on limited transects or short-duration sampling would be subject to bias and uncertainty. 


\section{Acknowledgements}

I would like to first thank my thesis supervisor Prof. Matthew Johnson for his guidance and support. His passion and enthusiasm for his work was contagious. He made every daunting task seem possible and I consider myself lucky to have worked with him. I hope to bring this attitude to every challenge ahead.

To everyone at the Energy and Emissions Research Lab, thank you for all the support. This group never let a dull day pass. Jay, Melina, Dave, Simon, Brad, Darcy, Steve, Brigid, Darcy, Brian, Nick, Nick, Rafid, Carol, Jordan, Zack, and Parvin: your support was absolutely indispensable and it would not have been the same without you all. Sometimes a group walk for coffee is the only way to solve your LabVIEW problems.

Thank you to everyone in the FlareNet family. It was a pleasure to have met and collaborated with so many people separated by so many miles.

A special thank you to my parents who, despite my protests during the writing of this thesis, always made sure I made it to family dinner on Sundays. You have made all of this possible, thank you. 


\section{Table of Contents}

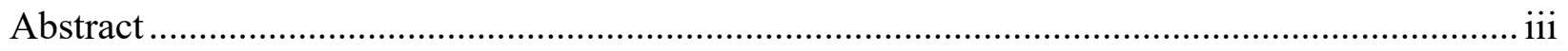

Acknowledgements .................................................................................................. iv

List of Tables ................................................................................................................. vii

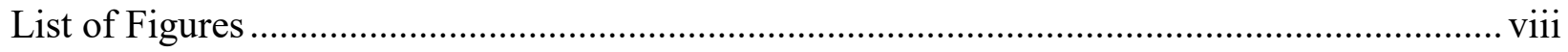

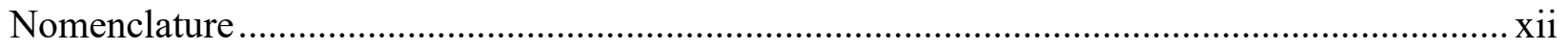

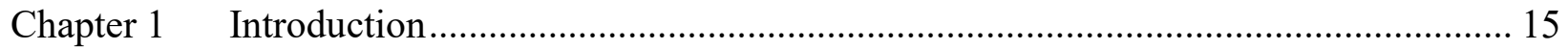

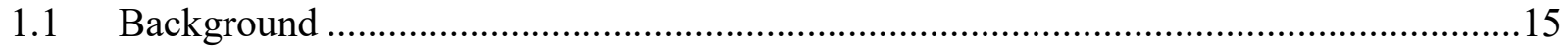

1.2 Recent Field Measurements of Flares ....................................................................

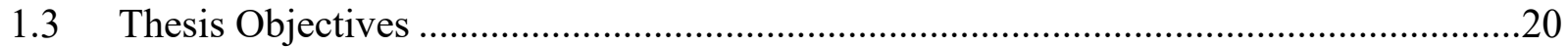

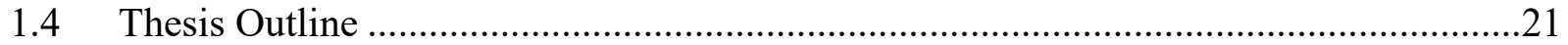

Chapter 2 Absorption Spectroscopy and Line-of-Sight Attenuation Theory ........................... 23

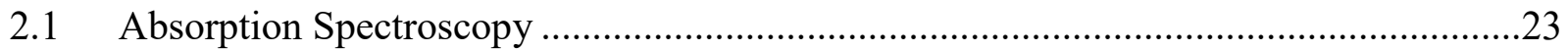

2.1.1 TDLAS in Non-Uniform Flow Environments .................................................. 27

2.1.2 Column Profile Fitting of Measured Spectra through Inhomogeneous Media....... 28

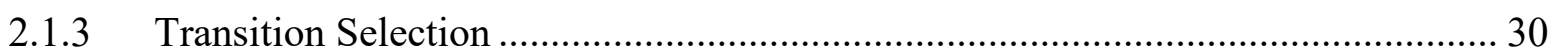

2.2 Soot Detection Using Line-of-Sight Attenuation (LOSA).............................................33

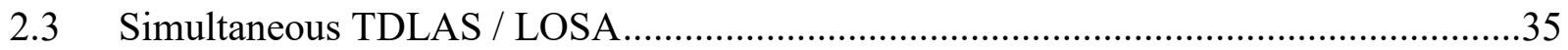

Chapter 3 Optical Technique Validation Simulations ......................................................... 38

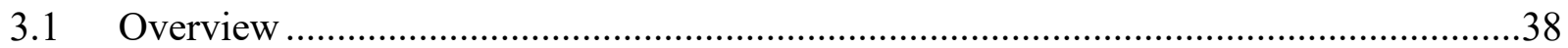

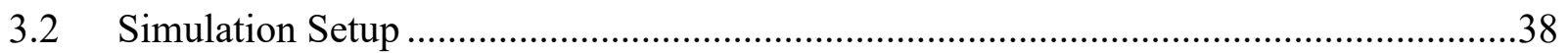

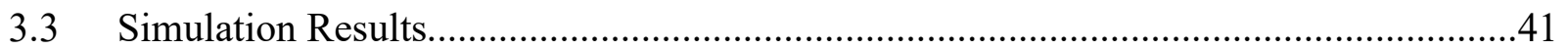

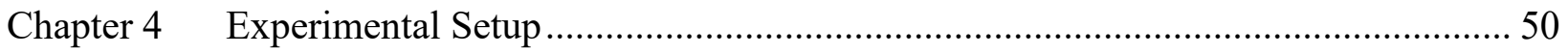

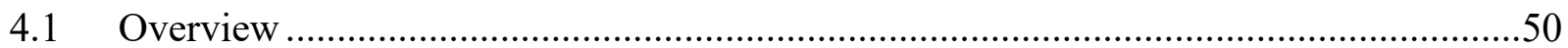

4.2 Laser Diode \& Laser Driving Systems ........................................................................50

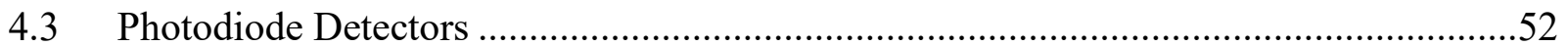

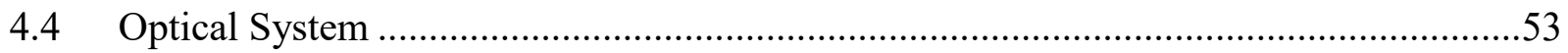

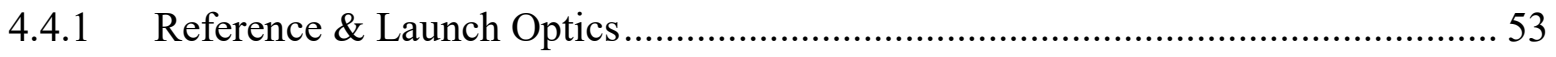

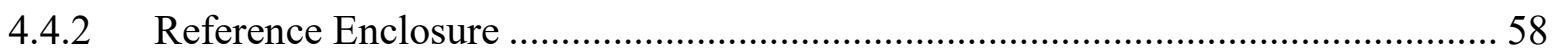

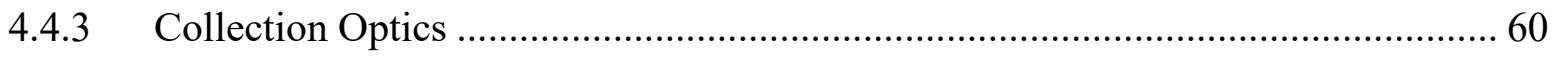

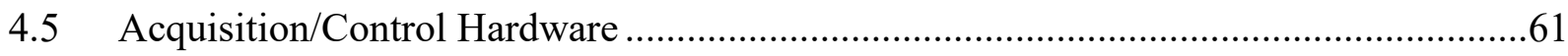

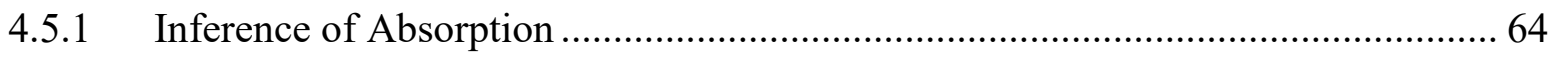

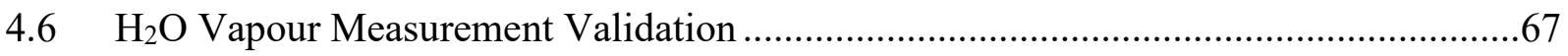




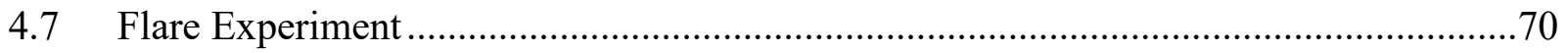

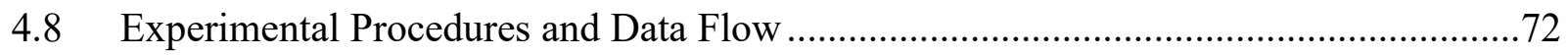

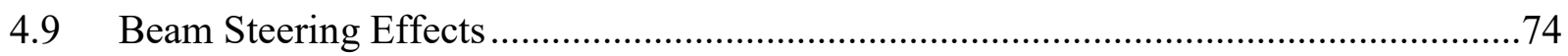

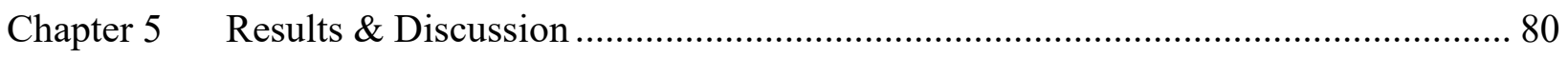

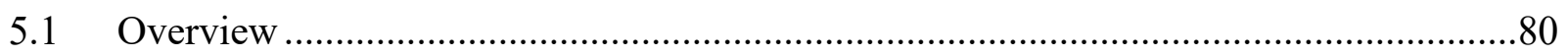

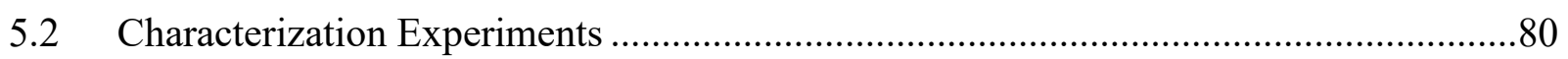

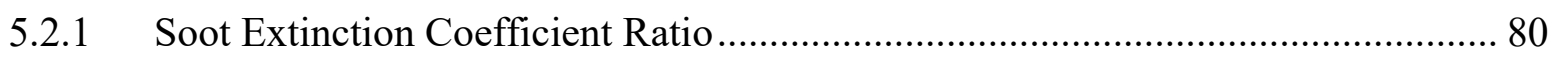

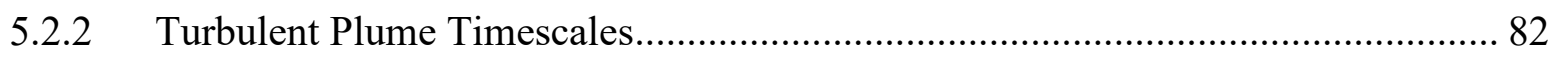

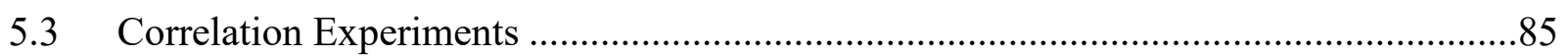

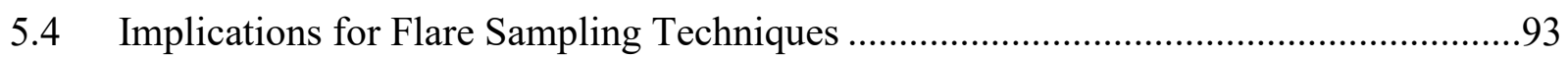

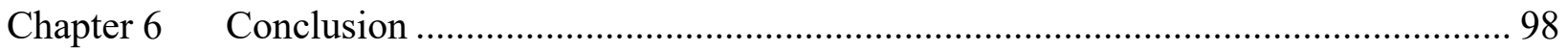

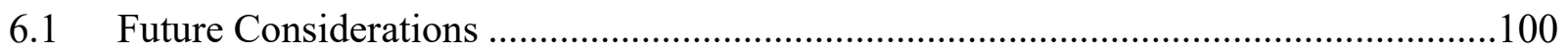

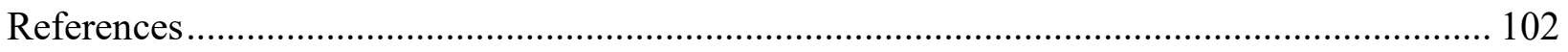

Appendix A Improved Spatial-Resolution LES Simulation Results ..................................... 108

Appendix B Time-Resolution of Spectral $\mathrm{H}_{2} \mathrm{O}$ Absorption Signals ..................................... 109

Appendix C Spectral Fitting Results of Flare Experiments................................................... 115

Appendix D Data Filtering and Inherent Measurement Skew .............................................. 118 


\section{List of Tables}

Table 2.1: Summary of soot extinction coefficient measurements with extinction coefficient ratios (ECRs) calculated for the wavelengths selected for measurement.

Table 3.1: Soot- $\mathrm{H}_{2} \mathrm{O}$ ratio statistics and correlation coefficient for different SSE cut-off values 47

Table 4.1: Major Components of Laser Driving Systems, Reference and Launch Optics .......... 54

Table 4.2: Major components of Collection Optics Assembly ............................................ 61

Table 4.3. Major components of the Acquisition \& Control Hardware Station ........................ 62

Table 4.4. Flare gas compositions in volume fraction [\%] studied in these experiments with their molecular weight $(\mathrm{MW})$, volumetric higher heating value $\left(\mathrm{HHV}_{\mathrm{V}}\right)$ and molar carbon-hydrogen

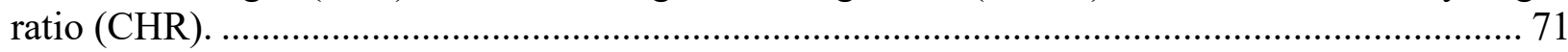

Table 4.5: Flow Conditions for Correlation Measurements .................................................... 72

Table 5.1: Summary of Flare Test Soot- $\mathrm{H}_{2} \mathrm{O}$ Ratio Results and Relevant Statistics.................. 92 


\section{List of Figures}

Figure 1.1: Comparison of black carbon emission factors as a function of $\mathrm{HHV}_{\mathrm{v}}$. Figure adapted from Conrad \& Johnson (2017). 18

Figure 2.1: Column distribution of temperature and $\mathrm{H} 2 \mathrm{O}$ volume fraction used to generate theoretical spectra for comparison with measured data. 29

Figure 2.2: (a) Transition linestrength for $\mathrm{H}_{2} \mathrm{O}$ transitions contributing to overall absorption spectra and (b) contributions of major and minor $\mathrm{H}_{2} \mathrm{O}$ peaks to overall absorption................................. 32

Figure 2.3: (a) Major $\mathrm{H}_{2} \mathrm{O}$ absorption peaks to be measured in flare plume experiments shown in isolation from minor $\mathrm{H}_{2} \mathrm{O}$ peaks with (b) corresponding temperature-dependent linestrengths. . 32

Figure 3.1: Instantaneous visualization of LES methane flare in crossflow (provided by Jeremy Thornock, University of Utah)............................................................................................. 39

Figure 3.2: Sample profile fitting result where (a) compares the column distribution spectra to the simulated measured spectra, and (b) and (c) compare the column distribution profiles with LOS simulation data for volume fraction and temperature, respectively........................................... 42

Figure 3.3: Relative error of $\mathrm{H}_{2} \mathrm{O}$ volume fraction results from simulated measured data compared to peak LOS temperature for each result. .............................................................................. 43

Figure 3.4: Relative error of path-averaged $\mathrm{H}_{2} \mathrm{O}$ compared to (a) true path-averaged temperatures and (b) estimated path-averaged temperatures calculated from column distribution results........ 44

Figure 3.5: Trend of increased relative error of $\mathrm{H}_{2} \mathrm{O}$ volume fractions with increase in SSE from

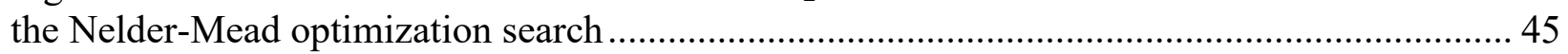

Figure 3.6: Scatter plots of soot and combustion-derived $\mathrm{H}_{2} \mathrm{O}$ volume fractions (a) before and (b) after the removal of results where peak line-of-sight temperatures exceed $800 \mathrm{~K}$........................ 46

Figure 3.7: Histogram of soot to combustion-derived $\mathrm{H}_{2} \mathrm{O}$ volume fraction ratio. The simulation results show a standard deviation of $6.92 \times 10^{-7}$, skewness of 1.08 and good agreement between mean values.

Figure 3.8: Sample of (a) spectral profile fitting results with intentionally-biased ambient $\mathrm{H}_{2} \mathrm{O}$ inputs with (b) and (c) showing the spatially distributed data against column fit results............. 49

Figure 4.1: Typical responsivity of PDA20CS Detectors. Adapted from Thorlabs (2017). ........ 52

Figure 4.2: Schematic of the laser driving systems and reference and launch optics................... 53

Figure 4.3: $\mathrm{H}_{2} \mathrm{O}$ absorption spectra observed in the $\mathrm{H}_{2} \mathrm{O}$ reference cell with the $1428 \mathrm{~nm}$ laser. The

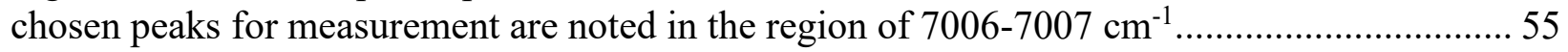

Figure 4.4: Methane absorption spectra observed in the reference cell for the $1654 \mathrm{~nm}$ laser. The measured wavenumber location was selected to avoid any light absorption from methane. ....... 56

Figure 4.5: $\mathrm{H}_{2} \mathrm{O}$ absorption observed in reference cell overlaid with the FRR signal.................. 57

Figure 4.6: (a) Thermally-Stable Reference Enclosure and (b) Enclosure internal view............. 59 
Figure 4.7: Schematic of collection optics. Dotted line indicates maximum extent of a steered beam. 60

Figure 4.8: Remote base station which includes laser driving systems, reference optics in a thermally-stable enclosure, and the PXI control and acquisition system. 62

Figure 4.9: (a) Example reference cell, FRR, and laser reference transmitted intensity measurements over one period of the triangle current-tuning waveform, and (b) absorption-free intensity estimate compared with reference cell signal. 65

Figure 4.10: Mirrored scan of two absorption peaks during one current-tuning period of the laser shown in terms of both absorption and transmission. 66

Figure 4.11: Lead-lag spectra compared on wavenumber basis for scan of Figure 4.10. The residual plot here shows the difference in absorption between lead and lag [\%]..... 66

Figure 4.12: Schematic of flow-through reference cell with heater and wrapped insulation to maintain temperature of heated vapour sample. 68

Figure 4.13: Samples of the spectral fit at three different temperature setpoints. Estimated temperatures closely agree with RTD-measured temperatures. 68

Figure 4.14: Time-varying results of $\mathrm{H}_{2} \mathrm{O}$ volume fraction and gas temperature from spectral

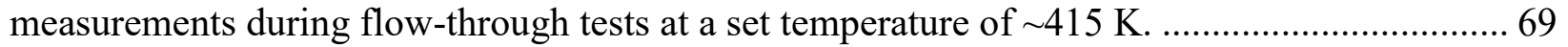

Figure 4.15: Schematic of Carleton University Flare Facility (CUFF). ................................. 71

Figure 4.16: Measured ambient $\mathrm{H}_{2} \mathrm{O}$ volume fraction measured between flare experiments ...... 73

Figure 4.17: Light attenuation due to beam steering and inferred SVF presence caused by the attenuation... 75

Figure 4.18: Theoretical $\mathrm{H}_{2} \mathrm{O}$ absorption at different gas conditions within the range of the $1428 \mathrm{~nm}$ laser. $\mathrm{H}_{2} \mathrm{O}$ absorption is at a minimum at $1426.686 \mathrm{~nm}\left(7009.25 \mathrm{~cm}^{-1}\right) \ldots \ldots \ldots \ldots \ldots \ldots . . .77$

Figure 4.19: Zoomed-in view of Figure 4.18 indicating the spectral region of minimum $\mathrm{H}_{2} \mathrm{O}$ absorption. Ref. A and B were used during CEM validation experiments to precisely position the laser output. 77

Figure 4.20: Difference in signal attenuation between laser signals which represents the asymmetry of beam alignment provoked by beam steering. 79

Figure 5.1: Distribution of estimated $\mathrm{Ke}$ ratio between 1428 and $1654 \mathrm{~nm}$ during ethylene flare test. The measured mean soot extinction coefficient ratio was 0.964 with a standard deviation of 0.065 . 82

Figure 5.2: Autocorrelation function of soot attenuation signals for seven 1-minute datasets. The mean effective timescale for measurements in the plume appears to be $\sim 0.38 \mathrm{~s}$. 83

Figure 5.3: Time-resolved soot attenuation signal over 10 seconds. Bulk fluctuations of soot attenuation most heavily weight the autocorrelation function and result in apparent timescales of $0.38 \mathrm{~s}$. 84

Figure 5.4: Time-resolved light soot attenuation signal over 15 milliseconds. During this time span, the $1428 \mathrm{~nm}$ laser $\left(\mathrm{H}_{2} \mathrm{O}\right.$ signal $)$ completes 3 sweeps. 84 
Figure 5.5: Sample $5 \mathrm{~ms}$ duration measurement of (a) $1428 \mathrm{~nm}\left(\mathrm{H}_{2} \mathrm{O}\right)$ and (b) $1654 \mathrm{~nm}$ (soot) laser signals through a Bakken flare plume. Time-resolved signals can be mapped to isolate spectral $\mathrm{H}_{2} \mathrm{O}$ absorption peaks from soot. 86

Figure 5.6: $\mathrm{H}_{2} \mathrm{O}$ absorption peaks in isolated, spectrally-resolved form compared to the column distribution result from the NM optimization search.

Figure 5.7: Histograms of estimated path-averaged temperatures in (a) Bakken, and (b) Ecuador fuel mixtures measurements. 88

Figure 5.8: Scatter plots of soot and combustion-derived $\mathrm{H}_{2} \mathrm{O}$ path-integrated volume fraction for (a) Bakken, and (b) Ecuador fuel mixtures measurements with coefficients of determination as noted. 89

Figure 5.9: Soot-to- $\mathrm{H}_{2} \mathrm{O}$ volume fraction ratios for Bakken flares.......................................... 89

Figure 5.10: Soot-to-Combustion $\mathrm{H}_{2} \mathrm{O}$ volume fraction ratios for Ecuador flares. 90

Figure 5.11: Combined Soot $-\mathrm{H}_{2} \mathrm{O}$ Ratios for the two fuel types considered along with their respective cumulative distribution functions (CDFs). 92

Figure 5.12: Distributions of sample mean for sample sets of size $N$ from Soot $-\mathrm{H}_{2} \mathrm{O}$ ratio results of the Bakken flare tests. 94

Figure 5.13: Distributions of sample mean for sample sets of size $N$ from Soot- $\mathrm{H}_{2} \mathrm{O}$ ratio results of the Ecuador flare tests. 94

Figure 5.14: (a) Standard error of the sample mean and (b) skew of the distribution with increased sample size. This indicates the convergence toward the population mean with increased sample size.

Figure A.1: Sample spectral fitting result from high spatial resolution simulation. Spectral fit (a) shows residuals on the same order as coarser simulation data in Chapter 3; (b) and (c) show comparison of line-of-sight data against effective column fit result. 108

Figure B.1: Varying synthetic $\mathrm{H}_{2} \mathrm{O}$ volume fraction and temperature data generated from measured soot attenuation signals. A 50-ms excerpt of the $1500 \mathrm{~ms}$ synthetic data shown. 110

Figure B.2: Time-resolved $\mathrm{H}_{2} \mathrm{O}$ absorption generated from theoretical fluctuations in Figure B.1 for a single $5 \mathrm{~ms}$ laser sweep period. 111

Figure B.3: Sample time-resolved $\mathrm{H}_{2} \mathrm{O}$ spectrum at $35-40 \mathrm{~ms}$ in the synthetic data. Minimal fluctuations of path avg. $\mathrm{H}_{2} \mathrm{O}$ return lead and lag spectra that agree closely. 111

Figure B.4: Sample time-resolved $\mathrm{H}_{2} \mathrm{O}$ spectrum at $85-90 \mathrm{~ms}$ in the synthetic data. Fluctuations of path avg. $\mathrm{H}_{2} \mathrm{O}$ return lead and lag spectra that differ however the average of lead and lag smooths out differences. 112

Figure B.5: Sample time-resolved $\mathrm{H}_{2} \mathrm{O}$ spectrum at 15-20 ms in the synthetic data. Substantial changes in path avg. $\mathrm{H}_{2} \mathrm{O}$ produces noticeably different lead and lag spectra. 112

Figure B.6: Column fit results to time-averaged spectra of Figure B.3, Figure B.4, and Figure B.5. Results return relative errors of $0.02 \%, 2.7 \%$, and $7.2 \%$, respectively. 113

Figure C.1: Comparison of inferred soot volume fraction in methane and hydrogen flares. No significant increase in SVF is apparent in the methane flare. 115 
Figure C.2: Sample attenuation signal on (a) $1428 \mathrm{~nm}\left(\mathrm{H}_{2} \mathrm{O}\right)$ and (b) $1654 \mathrm{~nm}$ (soot) lasers. A large light-attenuating soot structure is observed which peaks near the 800 index. 116

Figure C.3: Uncertainties in soot optical properties produces significant distortion of the isolated $\mathrm{H}_{2} \mathrm{O}$ absorption peaks. 117

Figure D.1: Sample spectral measurement from flow-through $\mathrm{H}_{2} \mathrm{O}$ vapour experiments conducted in Chapter 4. This sample represents an SSE of $1.08 \times 10^{-4}$, the maximum SSE accepted. 119

Figure D.2: Affect of changing the SSE cut-off value on the skewness of the Soot- $\mathrm{H}_{2} \mathrm{O}$ ratio results. 120

Figure D.3: Distribution of Soot- $\mathrm{H}_{2} \mathrm{O}$ ratio assuming normal distribution of parameters with errors estimated in previous work in this thesis. Inherent skewness of 0.37 was observed for the typical values of the two species. 


\section{Nomenclature}

\begin{tabular}{|c|c|c|c|c|}
\hline \multirow{2}{*}{ Latin } & \multirow[b]{2}{*}{ Description } & \multirow[b]{2}{*}{ Units } & \multicolumn{2}{|c|}{ First Use } \\
\hline & & & Eqn. & Page \\
\hline$A$ & Gain & - & $(4.1)$ & 52 \\
\hline$a$ & Fiber core radius & $\mathrm{m}$ & & 53 \\
\hline$c$ & Speed of light in a vacuum, $c_{0}=3 \times 10^{8} \mathrm{~m} / \mathrm{s}$ & $\mathrm{m} / \mathrm{s}$ & (4.2) & 24 \\
\hline$d$ & Fiber-ring resonator (FRR) fiber length & $\mathrm{m}$ & (4.2) & 56 \\
\hline$E$ & Soot absorption function & - & $(2.10)$ & 34 \\
\hline$E_{L} "$ & Lower state energy & $\mathrm{cm}^{-1}$ & $(2.3)$ & 24 \\
\hline$f_{v}$ & Soot volume fraction & - & (2.9) & 33 \\
\hline$h$ & Planck's constant, $h=6.626 \times 10^{-34} \mathrm{~m}^{2} \cdot \mathrm{kg} / \mathrm{s}$ & $\mathrm{m}^{2} \cdot \mathrm{kg} / \mathrm{s}$ & (2.3) & 24 \\
\hline I & Measured optical intensity & V & (2.1) & 23 \\
\hline$I_{0}$ & Absorption-free intensity & V & (2.1) & 23 \\
\hline$K_{e_{\lambda}}$ & Wavelength-dependent soot extinction coefficient & - & $(2.9)$ & 33 \\
\hline$k$ & Imaginary component of soot complex index of refraction & - & $(2.12)$ & 34 \\
\hline$k_{b}$ & Boltzmann's constant, $k_{b}=1.380658 \times 10^{-19} \mathrm{~kg} \cdot \mathrm{cm}^{2} /\left(\mathrm{s}^{2} \cdot \mathrm{K}\right)$ & $\mathrm{kg} \cdot \mathrm{cm}^{2} /\left(\mathrm{s}^{2} \cdot \mathrm{K}\right)$ & $(2.1)$ & 23 \\
\hline$m_{\lambda}$ & Soot complex index of refraction & - & & 34 \\
\hline$N A$ & Numerical aperture & - & & 53 \\
\hline$n$ & Real component of soot complex index of refraction & - & $(2.11)$ & 34 \\
\hline$n_{\text {air }}$ & HITRAN parameter: half-width temperature dependence & - & $(2.5)$ & 26 \\
\hline$P$ & Optical power & $\mathrm{W}$ & (4.1) & 52 \\
\hline$p$ & Pressure & $\mathrm{Pa}$ & (2.1) & 23 \\
\hline$p_{0}$ & HITRAN reference pressure, $p_{0}=101,325 \mathrm{~Pa}$ & $\mathrm{~Pa}$ & $(2.5)$ & 24 \\
\hline$Q_{R V}$ & $\begin{array}{l}\text { Total Internal Partial Sums (TIPS) function describing } \\
\text { statistical weightings of energy state levels of a molecule }\end{array}$ & - & $(2.3)$ & 24 \\
\hline$q$ & Species volume fraction & - & $(2.1)$ & 23 \\
\hline$q_{a m b}$ & Column Distribution: ambient $\mathrm{H}_{2} \mathrm{O}$ volume fraction & - & & 28 \\
\hline$q_{\mathrm{col}}$ & Column Distribution: column $\mathrm{H}_{2} \mathrm{O}$ volume fraction & - & & 28 \\
\hline$R_{g m}$ & Radius of gyration of soot aggregates & $\mathrm{m}$ & & 34 \\
\hline$R_{\text {load }}$ & Detector load resistance, $R_{\text {load }}=50 \Omega$ & $\Omega$ & (4.1) & 52 \\
\hline$R_{s}$ & Detector series resistance, $R_{S}=50 \Omega$ & $\Omega$ & $(4.1)$ & 52 \\
\hline$R_{\lambda}$ & Wavelength-dependent responsivity of photodiode element & $\mathrm{A} / \mathrm{W}$ & $(4.1)$ & 52 \\
\hline$S$ & HITRAN parameter: temperature-dependent linestrength & $\mathrm{cm} /$ molecule & (2.2) & 24 \\
\hline$s$ & Optical path position & $\mathrm{m}$ & (2.1) & 24 \\
\hline$T$ & Temperature & $\mathrm{K}$ & $(2.1)$ & 23 \\
\hline$T_{a m b}$ & Column Distribution: ambient temperature & $\mathrm{K}$ & & 28 \\
\hline$T_{\text {col }}$ & Column Distribution: column temperature & $\mathrm{K}$ & & 28 \\
\hline$T_{0}$ & HITRAN reference temperature, $T_{0}=296 \mathrm{~K}$ & $\mathrm{~K}$ & $(2.5)$ & 24 \\
\hline$V$ & V-parameter for single-mode transmission in a fiber & - & & 53 \\
\hline$V_{D C}$ & Voltage corresponding to dark current in detector & V & (4.1) & 52 \\
\hline$V_{\text {out }}$ & Detector output voltage & $\mathrm{V}$ & $(4.1)$ & 52 \\
\hline$w$ & Column Distribution: column width & $\mathrm{m}$ & & 28 \\
\hline$x_{p}$ & Soot particle size parameter & - & & 34 \\
\hline
\end{tabular}


Greek

Symbol

\begin{tabular}{ll}
\hline$\alpha$ & Absorption \\
$\alpha_{L}$ & HITRAN parameter: Lorentzian half-width half-maximum \\
$\alpha_{L_{\text {air }}}^{0}$ & HITRAN parameter: Air-broadened Lorentz half-width of a \\
$\alpha_{L_{\text {self }}}^{0}$ & spectral line for an absorbing species, at $T_{0}$ \\
$\delta s$ & HITRAN parameter: Self-broadened Lorentz half-width of a \\
$\lambda$ & spectral line for an absorbing species, at $T_{0}$ \\
$v$ & Node width in simulation domain \\
$v_{c}$ & Optical wavelength \\
$\rho_{S A_{\lambda}}$ & Wavenumber, inverse of wavelength \\
$\sigma$ & HITRAN parameter: zero-pressure wavecenter of a transition \\
$\sigma_{v_{c} j}$ & Soot scattering-to-absorption ratio \\
$\tau$ & Absorption cross-section \\
$\tau_{a m b}$ & Trsorption cross-section for transition at $v_{c}$ \\
$\tau_{c o l}$ & Column Distribution: ambient transmittance \\
$\tau_{d i s t}$ & Column Distribution: column transmittance \\
$\tau_{H_{2} O}$ & Column Distribution: transmittance of entire distribution \\
$\tau_{\text {soot }}$ & Transmissivity due to H ${ }_{2}$ O absorption \\
$\phi$ & Transmissivity due to soot attenuation \\
& HITRAN parameter: Absorption lineshape function
\end{tabular}

First Use

\begin{tabular}{cll} 
Units & Eqn. & Page \\
\hline- & $(2.7)$ & 26 \\
$\mathrm{~cm}^{-1} / \mathrm{atm}$ & $(2.4)$ & 26 \\
& & \\
$\mathrm{~cm}^{-1} / \mathrm{atm}$ & $(2.5)$ & 26 \\
& & \\
$\mathrm{~cm}^{-1} / \mathrm{atm}$ & $(2.5)$ & 26 \\
& & \\
$\mathrm{~m}$ & $(3.1)$ & 40 \\
$\mathrm{~nm}$ & $(2.9)$ & 23 \\
$\mathrm{~cm}^{-1}$ & $(2.1)$ & 23 \\
$\mathrm{~cm}^{-1}$ & $(2.2)$ & 24 \\
- & $(2.10)$ & 34 \\
$\mathrm{~cm}^{2} / \mathrm{molecule}^{2}$ & $(2.1)$ & 23 \\
$\mathrm{~cm}^{2} / \mathrm{molecule}^{\mathrm{m}}$ & $(2.6)$ & 26 \\
- & $(2.1)$ & 23 \\
- & $(2.8)$ & 29 \\
- & $(2.8)$ & 29 \\
- & $(2.8)$ & 29 \\
- & $(2.13)$ & 35 \\
- & $(2.13)$ & 33 \\
$\mathrm{~cm}$ & $(2.2)$ & 24
\end{tabular}

First Use

\begin{tabular}{lll} 
Acronym & Description & Page \\
\hline AR & Anti-Reflective & 61 \\
BK & Fuel mixture characteristic of Bakken oil field of the United States & 71 \\
CE/CCE & Combustion Efficiency/Carbon Conversion Efficiency & 16 \\
CEM & Controlled Evaporator and Mixer & 67 \\
CHR & Carbon-Hydrogen Ratio & 72 \\
CUFF & Carleton University Flare Facility & 21 \\
DA & Direct Absorption & 21 \\
DFB & Distributed Feedback laser diode & 28 \\
DRE & Destruction Removal Efficiency & 16 \\
EC & Fuel mixture characteristic of Ecuador's Orellana province & 71 \\
ECL & External Cavity Laser & 28 \\
ECR & Extinction Coefficient Ratio of soot & 36 \\
EF & Emission Factor & 16 \\
FC/APC & Fiber Coupled, Angled Physical Contact & 51 \\
FGEN & Frequency Generator & 63 \\
FPGA & Field-Programmable Gate Array & 63 \\
FRR & Fiber-Ring Resonator & 56 \\
FSR & Free Spectral Range & 56 \\
FTIR & Fourier-Transform Infrared Spectroscopy & 16 \\
FWHM & Full-Width Half-Maximum & 58 \\
GHG & Greenhouse Gas & 15 \\
GWP & Global Warming Potential & 15
\end{tabular}




\begin{tabular}{|c|c|c|}
\hline Acronym & Description & $\begin{array}{l}\text { First Use } \\
\text { Page }\end{array}$ \\
\hline $\mathrm{HHVv}$ & Volumetric Higher Heating Value & 17 \\
\hline HITRAN & High-Resolution Transmission Molecular Absorption Database & 24 \\
\hline HWHM & Half-Width Half-Maximum & 25 \\
\hline ICV & Image Correlation Velocimetry & 17 \\
\hline LabVIEW & Laboratory Virtual Instrument Engineering Workbench & 62 \\
\hline LDC & Laser Diode Controller & 54 \\
\hline LES & Large Eddy Simulation & 38 \\
\hline LOS & Line-Of-Sight & 16 \\
\hline LOSA & Line-Of-Sight Attenuation & 21 \\
\hline MIR & Mid-Infrared & 31 \\
\hline NASA & National Aeronautics and Space Administration & 15 \\
\hline NI & National Instruments & 62 \\
\hline NIR & Near-Infrared & 31 \\
\hline NM & Nelder-Mead simplex optimization search method & 29 \\
\hline NOAA & National Oceanic and Atmospheric Administration & 15 \\
\hline PXI & PCI eXtensions for Instrumentation & 62 \\
\hline RAM & Random Access Memory & 62 \\
\hline sky-LOSA & Line-Of-Sight Attenuation using Skylight & 17 \\
\hline SLPM & Standard Litres Per Minute (referenced at $0^{\circ} \mathrm{C}, 101325 \mathrm{~Pa}$ ) & 72 \\
\hline SMF & Single Mode Fiber & 53 \\
\hline SNR & Signal-Noise Ratio & 76 \\
\hline SRS & Stanford Research Systems & 51 \\
\hline SVF & Soot Volume Fraction & 33 \\
\hline TDLAS & Tunable Diode Laser Absorption Spectroscopy & 21 \\
\hline TEC & Thermoelectric Cooling & 59 \\
\hline TIPS & Total Internal Partition Sums Function & 24 \\
\hline UOG & Upstream Oil and Gas Industry & 15 \\
\hline VCSEL & Vertical-Cavity Surface-Emitting Laser & 28 \\
\hline WDM & Wavelength-division Multiplexing & 54 \\
\hline Chemical & & First Use \\
\hline Formula & Description & Page \\
\hline $\mathrm{BC}$ & Black Carbon & 15 \\
\hline $\mathrm{CH}_{4}$ & Methane & 71 \\
\hline $\mathrm{C}_{2} \mathrm{H}_{2}$ & Acetylene & 37 \\
\hline $\mathrm{C}_{2} \mathrm{H}_{4}$ & Ethylene & 37 \\
\hline $\mathrm{C}_{3} \mathrm{H}_{6}$ & Propylene & 37 \\
\hline $\mathrm{CO}$ & Carbon Monoxide & 15 \\
\hline $\mathrm{CO}_{2}$ & Carbon Dioxide & 15 \\
\hline $\mathrm{H}_{2}$ & Hydrogen & 71 \\
\hline $\mathrm{H}_{2} \mathrm{O}$ & Water & 20 \\
\hline $\mathrm{H}_{2} \mathrm{~S}$ & Hydrogen Sulfide & 97 \\
\hline InGaAs & Indium Gallium Arsenide & 52 \\
\hline $\mathrm{N}_{2}$ & Nitrogen & 55 \\
\hline $\mathrm{O}_{2}$ & Oxygen & 37 \\
\hline $\mathrm{SO}_{2}$ & Sulfur Dioxide & 97 \\
\hline
\end{tabular}




\section{Chapter 1 Introduction}

\subsection{Background}

In the upstream oil and gas industry (UOG), unwanted combustible gas is often disposed of through gas flaring. The gas is typically sent up a vertical stack and combusted in a turbulent diffusion flame open to atmosphere. Globally, over 140 billion $\mathrm{m}^{3}$ of gas was flared in 2012 based on estimates from NASA/NOAA Visible Infrared Imaging Radiometer Suite studies (Elvidge et al., 2015) and there has been little change in flared volumes in the years since (World Bank, 2018). Gas flaring is preferable to venting since the 20/100-year global warming potential (GWP) of methane, a common component of flare gas, is $96 / 34$ times higher than the $\mathrm{CO}_{2}$ that would be released during flaring (Gasser et al., 2017). However, incomplete combustion of the gas releases unwanted pollutants into the atmosphere (soot, $\mathrm{CO}$, uncombusted fuels, etc.), which can have their own climate and air quality impacts. Of these pollutants, black carbon (BC, the carbonaceous component of soot) has been suggested as the second most important atmospheric pollutant emission following $\mathrm{CO}_{2}$ (e.g., Bond et al., 2013; Jacobson, 2010). Atmospheric BC directly warms the atmosphere as it is a strong absorber of both in- and out-going radiation at all wavelengths; this is in contrast to greenhouse gases (GHGs) which tend to trap only the longer wavelength out-going radiation (U.S. EPA, 2012). BC that is deposited onto Arctic snow and ice has the additional effect of decreasing surface albedo (i.e., reflectance) and accelerating ice melting (Flanner, 2013; Sand et al., 2016). Black carbon has been identified to have a relatively short atmospheric lifetime (between 4-12 days) according to Cape et al. (2012) and is thus of particular interest to obtain nearterm climatic benefits. 
Field measurements of black carbon and other pollutant emissions from gas flaring operations are limited. The lack of direct pollutant emission measurements is due to the complexities in quantifying emissions in a turbulent, inhomogeneous, and unconfined plume which entrains air freely as it evolves. Emissions measurements made in the field generally fall into two categories, intrusive (extractive sampling) and non-intrusive (remote sensing). Both measurement types tend to characterize flare performance through destruction removal efficiency (DRE), carbon conversion efficiency (CCE) sometimes referred to as "combustion efficiency" (CE), or pollutant emission factors (EFs).

Extractive sampling methods use aspirated systems to pull gas samples into an instrument suite capable of measuring the constituent gas, liquid, and solid-phase species, as required. Measured relative concentrations of combustion-derived species are used to close a carbon massor molar-balance to estimate desired flare performance characteristics (Gvakharia et al., 2017; Herndon et al., 2012; Pohl et al., 1986; Strosher, 2000; Weyant et al., 2016). This approach relies on the assumption that the species in the extracted samples are well-mixed, and thus wellcorrelated, in the plume. However, if sufficient non-uniformities exist in the relative species concentrations, single-point or single-line extractive samples may not be sufficient to accurately determine flare performance.

Some non-intrusive measurement techniques may also suffer from an inability to account for inhomogeneities in relative species concentrations in the plume. Fourier Transform Infrared Spectroscopy (FTIR, both active and passive) has been used in a number of studies to remotely characterize flare performance (Blackwood, 2000; Gvakharia et al., 2017; Wormhoudt et al., 2012). FTIR methods estimate concentrations of species along a line-of-sight (LOS) through the plume by measuring characteristic absorption or emission spectra of the constituent gas species. 
These line-of-sight species concentrations can be used to estimate flare performance characteristics similar to the extractive methods. However, this approach also relies on the assumption of wellcorrelated combustion species within the observed plume.

An understanding of the degree to which combustion-species are correlated in space and in time is essential for quantifying the ability of different measurement techniques to make accurate measurements in a flare plume. Bounded estimates of degree of correlation between species and range of instantaneous fluctuations is important for developing consistent measurement protocols.

\subsection{Recent Field Measurements of Flares}

One of the few measurement techniques that does not rely on local species correlation is the imaging technique referred to as sky-LOSA (Line-of-Sight Attenuation by Skylight) that has been developed in recent years (Johnson et al., 2011, 2013). This technique uses narrow bandwidth grey-scale images coupled with image correlation velocimetry (ICV) to measure instantaneous mass emission rates of black carbon over a control volume. The instantaneous measurements of black carbon emission in the turbulent flare plume are typically recorded over tens of minutes and are used to derive a time-averaged emission factor for black carbon for the entire flare. Emission factors of black carbon have been recently reported using this technique from flares in Ecuador and Mexico (Conrad \& Johnson, 2017). Reported measurements have indicated a relationship between the volumetric higher heating value $\left(\mathrm{HHV}_{\mathrm{v}}\right)$ of the fuel and black carbon emission factor. These measurements have also shown that instantaneous emission rates tend to have skewed distributions about the mean, such that the average is sensitive to occasional bursts of highemission while there is a also higher probability of detecting low emission rates at any moment in time (Conrad \& Johnson, 2017). 
Weyant et al. (2016) estimated black carbon emission factors by navigating an aircraft outfitted with aspirated sampling systems through flare plumes in the Bakken region of the United States. Up to six transects were made through the plume (duration of 1-2 seconds each) to collect samples, and relative species concentrations of $\mathrm{CO}_{2}$, black carbon, and methane were used to close a carbon mass-balance and estimate $\mathrm{BC}$ emission factors. The authors noted that estimated emission factors spanned two orders of magnitude and flares resampled on subsequent days did not maintain constant EFs (in one case subsequent measurements yielded an EF 12 times higher). A study by Gvakharia et al. (2017), using the same methodology, obtained EFs spanning an order of magnitude and also reported emission factors varying for the same flare on subsequent days.

Emission factor results from Weyant et al. (2016) stand in stark contrast to EF estimates from sky-LOSA measurements of (Conrad \& Johnson, 2017) and laboratory flares measured by McEwen \& Johnson (2012). Figure 1.1 shows the apparent discrepancy between the measurements on the basis of $\mathrm{HHV}_{\mathrm{v}}$. At best, there is a 2.2 times discrepancy between the linear fit of results from Conrad \& Johnson (2017) and McEwen \& Johnson (2012) compared with Weyant et al. (2016); at worst the discrepancy is nearly a factor of 1500 .
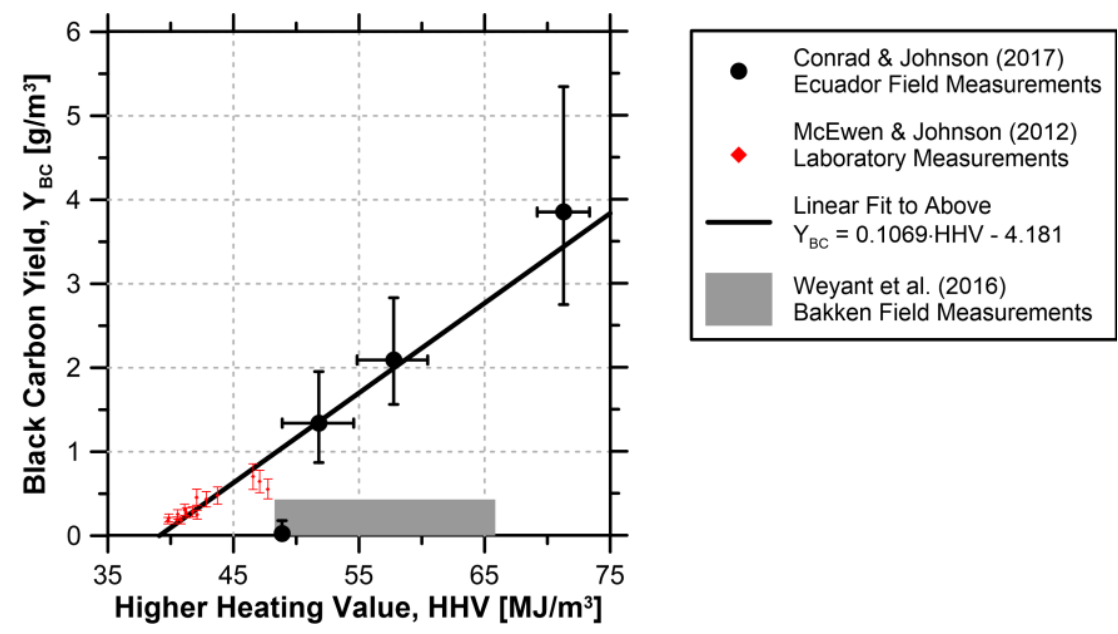

Figure 1.1: Comparison of black carbon emission factors as a function of $\mathrm{HHV}_{\mathrm{v}}$. Figure adapted from Conrad \& Johnson (2017). 
Although there are other potential causes for the discrepancy between the measurement techniques, the potential uncertainty of species correlation in the plume is not discussed by Weyant et al. (2016). As noted above, Conrad \& Johnson (2017) report that in their measurements, the timeaveraged black carbon emissions are heavily weighted by short, localized bursts. In one flare measurement, $10 \%$ of instantaneous emission rate data accounted for $56 \%$ of the total emission. If the black carbon is not well-correlated with the gaseous carbon species during these soot bursts, this could imply that a few extractive samples (such as those taken in the aircraft measurements) are unlikely to capture a representative overall mean emission rate.

In a study by Wormhoudt et al. (2012), a comparison was made between remote sensing (passive-FTIR) and extractive sampling on an open-air $80 \%$ propylene, $20 \%$ Tulsa natural gas steam-assisted flare as part of the 2010 Comprehensive Flare Study in Texas. Although some disagreement was observed in the estimates of combustion efficiency from the two methods, the researchers reported that the discrepancy was mainly accounted for by different detection capabilities of the two instruments for the uncombusted fuel. It was noted that the strongest correlation anticipated in the plume would be between the two constituent fuels (propylene and methane). However, it was reported that the propylene was always found in slightly lower amounts suggesting the preferential combusting of propylene relative to methane. This apparent preferential combustion of constituents of the fuel could be a potential source of inhomogeneities in relative species concentrations in the plume.

Although the study by Wormhoudt et al. (2012) generally reported agreement between the extractive and remote sensing measurements, the measurements were also heavily averaged. The extractive sampling system drew $1 \mathrm{~m}^{3} / \mathrm{s}$ of gas from the plume, as reported by Knighton et al. (2012), which was mixed, specifically to avoid issues related to inhomogeneities of relative species 
concentrations. The suite of instruments in the extractive sampling system produced concentration data at $1 \mathrm{~Hz}$. The remote sensing of the passive FTIR may have also avoided issues related to inhomogeneities in relative species concentration as it had a $0.3 \mathrm{~m}$ diameter field of view and a sampling rate of $1 \mathrm{~Hz}$. The large observed area and relatively low measurement rates may help to average out the inhomogeneities in relative species concentrations.

Often-cited studies such as McDaniel (1983), which forms the basis for a large portion of black carbon inventories in both the United States and Canada, and field experiments by Strosher (2000) that measured combustion efficiency of flares, both employed point-sampling techniques. Neither study explicitly considered the potential for fluctuations in relative species concentrations to introduce uncertainty in the mass-balance calculations and affect results.

Local fluctuations of relative species concentrations have the potential to hamper the performance of extractive sampling and remote sensing techniques if sufficient inhomogeneities exist in turbulent flare plumes. Although the remote and extractive sampling measurement studies referred to in this section may have performed sufficient measurements to yield representative results, without an understanding of local species correlation it is difficult to be certain. If relative species concentrations fluctuate sufficiently then there may be a need for unified measurement protocols to ensure all measurement techniques tend toward representative values and/or have accurate bounds on their estimates.

\subsection{Thesis Objectives}

The objective of this thesis was to develop an optical measurement system capable of making simultaneous, path-averaged in-situ measurements of $\mathrm{H}_{2} \mathrm{O}$ vapour and soot in turbulent flare plumes to inspect temporally-resolved species correlations. The system relies on Line-Of-Sight 
Attenuation (LOSA) and Tunable Diode Laser Absorption Spectroscopy (TDLAS) techniques for soot and $\mathrm{H}_{2} \mathrm{O}$ detection, respectively. The dual detection methods are modelled after a study by Wu et al. (2017), which allows for the decoupling of soot attenuation and spectral $\mathrm{H}_{2} \mathrm{O}$ absorption signals. The system implements a spectral-fitting technique inspired by (Liu et al., 2007a) to interpret measured spectra from a non-uniform flow-field and extract pseudo-mean $\mathrm{H}_{2} \mathrm{O}$ volume fractions. This technique, although path-averaged through the plume, will have the advantage of inspecting the plume along a $2-\mathrm{mm}$ nominal diameter chord through a plume at a sampling rate of $200 \mathrm{~Hz}$. This is a much higher data rate and finer spatial resolution than that achieved with passiveFTIR techniques such as the study by Wormhoudt et al. (2012) which had an imaging diameter of $0.3 \mathrm{~m}$ and a $1 \mathrm{~Hz}$ sampling rate.

The developed spectroscopic analysis techniques were first validated using simulated signals generated from temperature/concentration profiles extracted from large eddy simulation data of methane flares in cross-flow. The completed optical system was used to make a measurement of relative soot optical properties as a necessary step before simultaneous detection experiments were conducted. Simultaneous measurements of $\mathrm{H}_{2} \mathrm{O}$ and soot were made through lab-scale turbulent flare plumes generated at the Carleton University Flare Facility (CUFF). Observed species correlations in the path-averaged measurement were used to assess the potential for uncertainty or bias in extractive or remote sampling systems.

\subsection{Thesis Outline}

Chapter 2 presents the optical techniques involved in making simultaneous measurements of $\mathrm{H}_{2} \mathrm{O}$ and soot volume fractions through direct absorption (DA) spectroscopic measurements and broadband light attenuation, respectively. Chapter 3 demonstrates the expected performance and 
limitations of the developed optical techniques in a large eddy simulation of a methane flare. In Chapter 4, the experimental setup and signal processing methods are discussed along with experimental system validation measurements. Chapter 5 presents the results of the relative soot optical property measurements and results from the simultaneous species concentration measurements. The results of the simultaneous measurements are used to illustrate the potential detrimental effects that species correlation could present for many flare performance measurement techniques. Chapter 6 summarizes the findings of the thesis and makes recommendations for future work and system improvements. 


\section{Chapter 2 Absorption Spectroscopy and Line-of-Sight Attenuation Theory}

This chapter discusses the underlying optical theory for simultaneous soot and $\mathrm{H}_{2} \mathrm{O}$ vapour measurement in turbulent flare plumes. Tunable diode laser absorption spectroscopy (TDLAS) is reviewed and specific techniques for spectral analysis in harsh/non-uniform flow-fields are presented. In addition, line-of-sight attenuation (LOSA) techniques for soot detection and the isolation of broadband soot attenuation and $\mathrm{H}_{2} \mathrm{O}$ spectral signals are discussed.

\subsection{Absorption Spectroscopy}

Absorption spectroscopy is the method of exciting target molecules in a sample medium with radiation and inspecting the optical transmissivity of the sample over some wavelength range corresponding to the molecule's unique ro-vibrational patterns. The governing equation of absorption spectroscopy is Beer-Lambert's law, shown in Eq. (2.1), which relates the incident optical intensity $I_{0}[\mathrm{~V}]$ to the transmitted intensity $I[\mathrm{~V}]$,

$$
\tau(v)=\frac{I}{I_{0}}=\exp \left(-\int_{s} \frac{q p}{k_{b} T} \sigma(p, T, \mathrm{q}, v) d s\right)
$$

where $\tau(v)[-]$ is the optical transmissivity evaluated at some wavenumber, $v\left[\mathrm{~cm}^{-1}\right]$ and the wavenumber is the inverse of the optical wavelength $\lambda[\mathrm{nm}] . \tau(v)$ is related to gas properties where $q[-]$ is the volume fraction of the absorbing species, $p[\mathrm{~Pa}]$ is the pressure, $k_{b}$ is Boltzmann's constant $\left[1.38064852 \times 10^{-19}\left(\mathrm{~kg} \cdot \mathrm{cm}^{2}\right) /\left(\mathrm{s}^{2} \cdot \mathrm{K}\right)\right], T[\mathrm{~K}]$ is the gas temperature, and $\sigma(p, T, \mathrm{q}, v)\left[\mathrm{cm}^{2} /\right.$ molecule $]$ is the absorption cross-section evaluated at some wavenumber. The 
equation is computed by integrating over the optical path, $s[\mathrm{~m}]$. The absorption cross-section of a single spectral peak centered at some wavecenter $v_{c}$ can be decomposed as shown in Eq. (2.2),

$$
\sigma(p, T, q, v)=S(T) \phi\left(p, T, q, v-v_{c}\right)
$$

where $S(T)\left[\mathrm{cm} /\right.$ molecule] is the transition linestrength and $\phi\left(p, T, q, v-v_{c}\right)[\mathrm{cm}]$ is the transition lineshape. The temperature-dependent linestrength of an individual spectral transition at $v_{c}$ can be calculated using Eq. (2.3),

$$
S(T)=S\left(T_{0}\right) \frac{Q_{R V}\left(T_{0}\right)}{Q_{R V}(T)} \frac{\exp \left(-h c E_{L}^{\prime \prime} / k_{b} T\right)}{\exp \left(-h c E_{L}^{\prime \prime} / k_{b} T_{0}\right)} \frac{\left(1-\exp \left(-h c v_{c} / k_{b} T\right)\right)}{\left(1-\exp \left(-h c v_{c} / k_{b} T_{0}\right)\right)}
$$

where $h$ is Planck's constant $\left[6.626 \times 10^{-34} \mathrm{~m}^{2} \cdot \mathrm{kg} / \mathrm{s}\right], E_{L}{ }^{\prime \prime}\left[\mathrm{cm}^{-1}\right]$ is the lower state energy, $c$ is the speed of light in a vacuum $\left[3 \times 10^{8} \mathrm{~m} / \mathrm{s}\right]$, and $Q_{R V}[-]$ is the total internal partition sums (TIPS) function which describes statistical weights of state energy levels of the molecule (i.e., rotational, vibrational, etc.) (Fischer, 2003). Equation (2.2) establishes a scaling of a reference linestrength taken at $p_{0}=101.325 \mathrm{kPa}$ and $T_{0}=296 \mathrm{~K}$ from the 2008 High-resolution Transmission molecular absorption (HITRAN) spectral database (Rothman et al., 2009). HITRAN is a commonly used database used to simulate atmospheric absorption/emission phenomena.

In a vacuum, light absorption of the target molecule would occur only at zero-pressure wavecenter, $v_{c}$. However, in the presence of other molecules, molecular motions and collisions cause these absorption transitions to be spectrally broadened into absorption peaks. These effects are encapsulated in the lineshape parameter, $\phi$. The absorption lineshape describes both homogeneous and inhomogeneous broadening mechanisms. The Gaussian lineshape models the inhomogeneous Doppler (temperature) broadening and the Lorentz lineshape models the 
homogeneous natural and collisional (pressure) broadening (Hanson et al., 2016; Schiff et al., 1994). The Voigt lineshape is the convolution of Lorentz and Gaussian lineshapes and can be used in cases where both broadening phenomena are significant at the target gas conditions.

For media near ambient pressure and temperature, the collisional broadening effects tend to dominate over temperature broadening allowing the Lorentz lineshape to be used (Schiff et al., 1994). This reduces the computational costs that would be demanded by the Voigt profile. In the present work, the Lorentz profile was used since the temperature broadening contributes roughly four orders of magnitude less absorption than the natural and collisional broadening. The Lorentz lineshape of a transition depends on gas temperature, gas partial pressure (self-interaction, or selfbroadening), and total pressure (foreign gas broadening). The HITRAN database includes both self- and air-broadened Lorentz parameters. If the target gas is assumed to be similar to air in composition, then it is possible to assume the use of the air-broadening term. Equation (2.4) describes the Lorentzian lineshape as,

$$
\phi\left(v-v_{c}\right)=\frac{1}{\pi} \frac{\alpha_{L}}{\left(v-v_{c}\right)^{2}+\alpha_{L}^{2}}
$$

where $\alpha_{L}\left[\mathrm{~cm}^{-1} / \mathrm{atm}\right.$ at $\left.T\right]$ is the Lorentzian half-width half-maximum (HWHM) of the peak (Gordley et al., 1994; Hanson et al., 2016). This can be further broken down in Eq. (2.5) following HITRAN nomenclature to address both self- and air-broadening as well as pressure and temperature scaling,

$$
\alpha_{L}=\left((1-q) \alpha_{L_{\text {air }}}^{0}+q \alpha_{L_{\text {self }}}^{0}\right) \frac{p}{p_{0}}\left(\frac{T_{0}}{T}\right)^{n_{\text {air }}}
$$


where $q[-]$ is the volume fraction of the target gas species, $\alpha_{L_{\text {air }}}^{0}$ and $\alpha_{L_{\text {self }}}^{0}\left[\mathrm{~cm}^{-1} / \mathrm{atm}\right.$ at $\left.T_{0}\right]$ are the air- and self-broadening Lorentz HWHM coefficients, and $n_{\text {air }}[-]$ is the half-width temperature dependence of the air-broadened half-width.

In general, there is overlap between the broadened spectral transitions of the target molecules and those of other species in the medium. To describe the transmittance of radiation through some multicomponent medium, the effect of all spectral transitions from all relevant species must be considered (Gordley et al., 1994). The general form for the transmissivity considering multiple species is shown in Eq. (2.6),

$$
\tau(v)=\frac{I}{I_{0}}=\exp \left(\int_{s} \sum_{j} \sum_{i} \frac{q_{j} p}{k T} \sigma_{v_{c_{i}} j}\left(p, T, q_{j}, v\right) d s\right)
$$

where $\sigma_{v_{c i} j}$ is the cross-section of the $i^{\text {th }}$ spectral peak of the $j^{\text {th }}$ gas species all integrated over the optical path, $s$.

Equations (2.1) and (2.6) can also be described in terms of light absorption $\alpha[-]$ which is shown in Eq.(2.7),

$$
\alpha(v)=1-\tau(v)
$$

In Eq. (2.7), the gas properties must be integrated along the optical path, s. In controlled environments where gas properties are known and can be assumed uniform, the integral can be greatly simplified. However, measurements through turbulent flare plumes do not have uniform distributions of species volume fractions and temperature along the line-of-sight (LOS) and these distributions are constantly changing in time. To use Eq. (2.7) to yield meaningful temperature 
and volume fraction information, additional theory must be developed to infer information about the distribution of gas temperature and target molecule volume fraction along the LOS.

\subsubsection{TDLAS in Non-Uniform Flow Environments}

Because the magnitude of light absorption depends not only on target species volume fraction, but also on temperature and pressure, it is possible to extract meaningful information about multiple gas properties simultaneously. There is a wealth of studies that inspect two absorption transitions to determine an effective (pseudo-mean) temperature and volume fraction in a relatively wellmixed flow-field. The technique, referred to as integrated absorption or absorbance ratio involves scanning two absorption transitions, integrating the area under each peak, and comparing with theory to determine a path-averaged species volume fraction. The pair of transitions must possess sufficiently different lower state energies since this is a proxy for temperature scaling. The temperature scaling must also be distinguishable from scaling due to changes in volume fraction. This method has been used to make measurements in tube furnaces (Liu et al., 2004; Shao et al., 2009; Wu et al., 2014) and in flames and combustor exhaust streams (Farooq et al., 2008; Fotia et al., 2015; Liu et al.; Allen, 1998; Zhou et al., 2003) where a single volume fraction and single temperature can be used to describe the inspected gas. However, this approach cannot account for large temperature and volume fraction fluctuations anticipated in flare plumes.

Integrated absorption methods can be expanded upon by probing more than two transitions and performing a linear least-squares regression to determine an approximate solution. This approach assumes a set of temperature and volume fraction bins and estimates the binned distribution that produces the best-matching spectra to measured data. The technique has been used in controlled furnace experiments (Sanders et al., 2001), combustor exhaust (Liu et al., 2005),

and in laminar flames (Liu et al., 2013; Zhang et al., 2016). The approach is very powerful when 
many transitions are scanned, which is why most studies utilize External Cavity Lasers (ECL), Vertical Cavity Surface-Emitting Lasers (VCSEL), or multiple distributed feedback (DFB) diode lasers which are able to inspect many absorption peaks. This technique can be further extended by assuming a functional form of the distribution of gas properties which is solved through linear least squares regression to fix the distribution parameters. This technique, called profile-fitting, was successfully demonstrated by Liu et al. (2007) in laminar flames. Unfortunately, this approach relies on being able to fully scan across each feature to perform the integration which is difficult for a single DFB laser diode as used for this experiment.

Where the integrated absorbance technique is not possible, individual points on the absorption features can be used instead. This assumes that the lineshape function accurately describes the broadening within the expected test conditions. This approach, a modified profilefitting technique using a column or "tophat" distribution, was shown in preliminary simulations to most accurately return path-averaged temperatures and species volume fractions, and is the method expanded upon in this thesis.

\subsubsection{Column Profile Fitting of Measured Spectra through Inhomogeneous Media}

The column or tophat distribution is shown in Figure 2.1. The distribution has three independent variables (column width $w$, temperature $T_{c o l}$, and volume fraction $q_{c o l}$ ) along with two inputs (ambient temperature $T_{a m b}$, and volume fraction $q_{a m b}$ ) which must be estimated. 

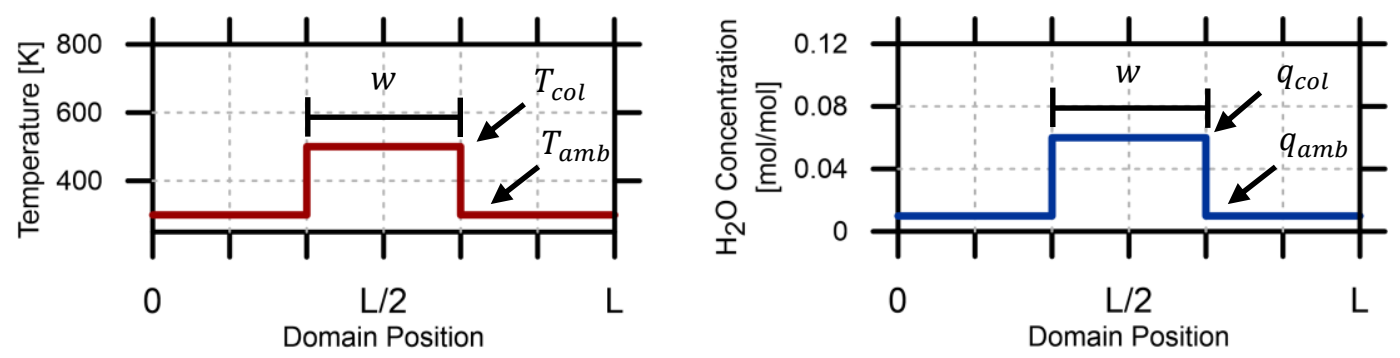

Figure 2.1: Column distribution of temperature and $\mathrm{H}_{2} \mathrm{O}$ volume fraction used to generate theoretical spectra for comparison with measured data.

The properties of the column distribution can be used to produce a theoretical absorption spectrum using Eq. (2.8),

$$
\tau_{d i s t}=\tau_{a m b} \tau_{c o l}
$$

where $\tau_{a m b}$ and $\tau_{c o l}[-]$ are the transmissivities of the ambient and column components of the distribution which are determined using the ambient and column temperatures and volume fractions, respectively. The theoretical spectrum of this distribution can then be made to match a measured spectrum by fitting the distribution parameter set $\left\{w, T_{c o l}, q_{c o l}\right\}$. The distribution parameters are selected through an optimization algorithm that minimizes absorption residuals between the theoretical and measured spectra. In the present work the optimization search was conducted using a Nelder-Mead downhill simplex method.

The Nelder-Mead (NM) optimization scheme is a compact and computationally efficient method for evaluating the minimum of a function of $n$ variables (Nelder \& Mead, 1965). In this case, the function is simply the difference between the measured spectral absorption and theoretical absorption generated by the assumed column distribution. Formally, the NM scheme attempts to find the set $\left\{w, T_{c o l}, q_{c o l}\right\}$ of $\min \sum_{i=1}^{N}\left(\tau_{\text {meas }}\left(v_{i}\right)-\tau_{\text {col }}\left(v_{i}\right)\right)^{2}$ for $N$ points along the spectral measurement. 
This approach requires accurate spectral parameters from the HITRAN database. In the present experiment, expected temperatures are not anticipated to greatly exceed $500 \mathrm{~K}$ (Poudenx et al., 2004). A study by Liu et al. (2007), who inspected $\mathrm{H}_{2} \mathrm{O}$ vapour transitions at 1388 and $1345 \mathrm{~nm}$ (relatively close to the working wavelength at $1428 \mathrm{~nm}$ ), found HITRAN parameters for those transitions to be in good agreement with measured values at tests conditions up to $1050 \mathrm{~K}$ and $25 \mathrm{~atm}$. Since the gas conditions in this work are much closer to the HITRAN reference conditions of $296 \mathrm{~K}$ and $1 \mathrm{~atm}$, it is anticipated that the HITRAN parameters will be sufficiently accurate. Although parameters from HITRAN may be accurate, Rieker et al. (2009) stress that measurement accuracy is heavily dependent on the intelligent selection of the absorption transitions to be measured. The transitions must be selected to guarantee sufficient sensitivity to the properties to be measured without interference.

\subsubsection{Transition Selection}

Selection of target transitions is a critical step to making a spectroscopic measurement. Important considerations include:

- Absorption strength: high absorption negates the optically thin assumption $(\alpha<0.1)$, but low absorption becomes increasingly difficult to detect

- Isolated transitions: too many overlapping transitions becomes difficult to model and overlap of other species' transitions hinders profile-fitting

- Lower state energies: two $\mathrm{H}_{2} \mathrm{O}$ vapour absorption peaks must possess sufficiently different lower state energies to be able to distinguish temperature fluctuations from volume fraction fluctuations in a profile-fitting search

- Equipment availability: individual diodes must be available at a sufficient power output; fiber optics, lenses, etc. must also be available 
After considering many potential transitions from HITRAN, two absorption peaks for $\mathrm{H}_{2} \mathrm{O}$, centered at $7006.12 \mathrm{~cm}^{-1}(1427.32 \mathrm{~nm})$ and $7007.03 \mathrm{~cm}^{-1}(1427.14 \mathrm{~nm})$, were selected; these transitions are related to combinational vibrational bands of $\left(2 v_{2}+v_{3}\right)$ and $\left(v_{1}+v_{3}\right)$, respectively. These transitions appeared to have sufficient absorption to measure in the lab, were isolated from other species transitions, and possessed the unique characteristic of having opposite scaling trends with increased temperature over the expected range ( $300 \mathrm{~K}$ to $800 \mathrm{~K})$. The expected temperatures in the flare plumes are well below species reaction temperatures (e.g., adiabatic flame temperature of methane at $\sim 2200 \mathrm{~K}$, thermal $\mathrm{NO}_{\mathrm{x}}$ formation above $\sim 1600 \mathrm{~K}$ ) (Turns, 2000; U.S. EPA, 1999) meaning no major chemical reactions were occurring at the measurement height above the flare.

Figure 2.2 shows a sample transition line survey of the two main absorption peaks against a background of minor $\mathrm{H}_{2} \mathrm{O}$ peaks. Other likely combustion species such as $\mathrm{CO}_{2}, \mathrm{CO}$, and methane are not shown as their theoretical absorption is at least 4 orders of magnitude lower in the selected wavelength range. Figure 2.3 shows the two major $\mathrm{H}_{2} \mathrm{O}$ transitions and their corresponding linestrengths as a function of temperature. The opposing temperature trends of the transitions makes it possible to differentiate between changes in $\mathrm{H}_{2} \mathrm{O}$ volume fraction and temperature. With the $\mathrm{H}_{2} \mathrm{O}$ absorption peaks near $1428 \mathrm{~nm}$ and the selection of the soot detection wavelength at $1654 \mathrm{~nm}$, it was possible to purchase commercially available fiber optics and lenses made to accommodate both wavelengths.

Although measurements of $\mathrm{CO}_{2}$ would be ideal for comparison to other flare performance measurement techniques, $\mathrm{CO}_{2}$ transitions were deemed infeasible due to many of the considerations listed above. $\mathrm{CO}_{2}$ absorption transitions in the near infrared (NIR) are not sufficiently strong to be detectable. Strong $\mathrm{CO}_{2}$ transitions in the mid-infrared (MIR) cannot be fiber-coupled due to attenuation in the fiber, which would require the laser to be mounted in close 
proximity to the flare. For these reasons, $\mathrm{H}_{2} \mathrm{O}$ transitions were selected for the measurements in this thesis.

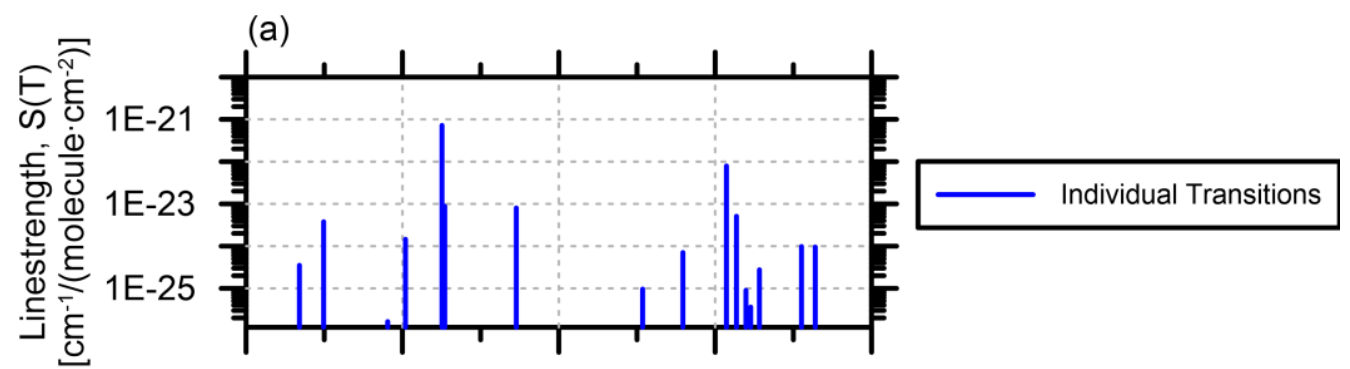

(b)

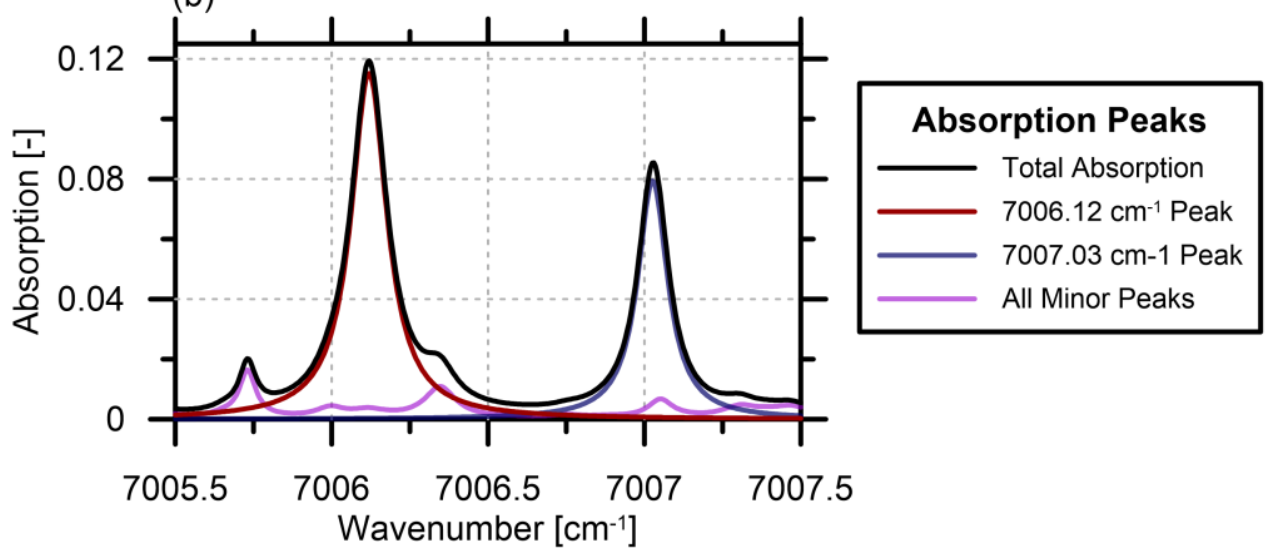

Figure 2.2: (a) Transition linestrength for $\mathrm{H}_{2} \mathrm{O}$ transitions contributing to overall absorption spectra and (b) contributions of major and minor $\mathrm{H}_{2} \mathrm{O}$ peaks to overall absorption.

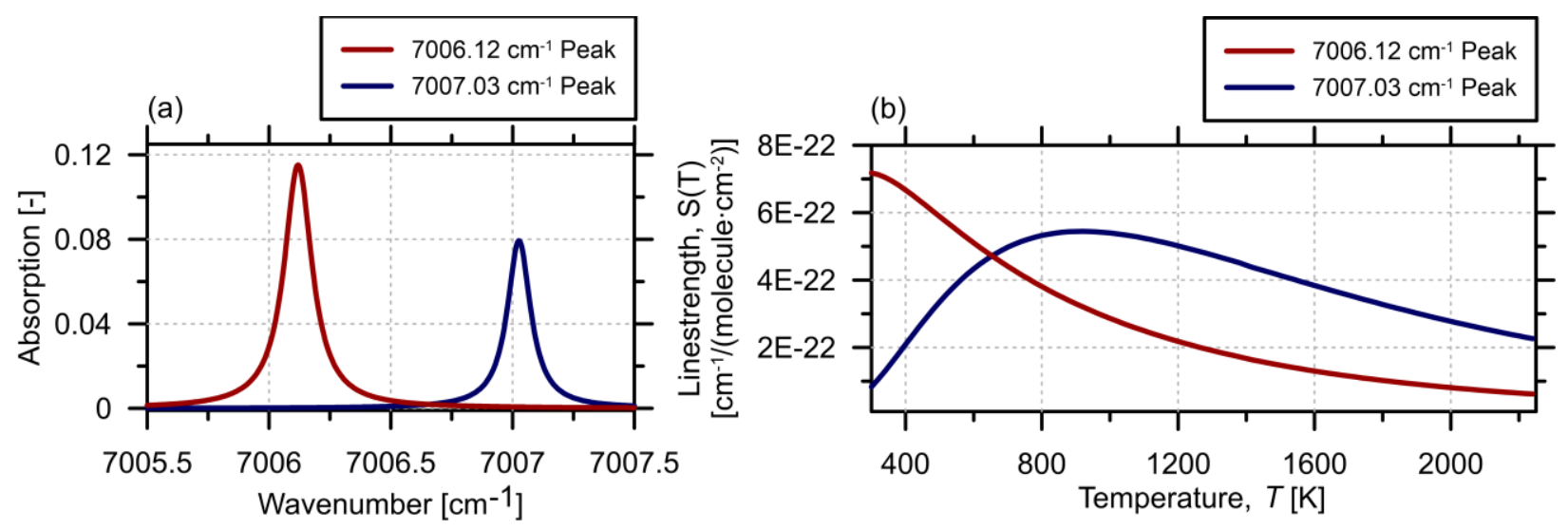

Figure 2.3: (a) Major $\mathrm{H}_{2} \mathrm{O}$ absorption peaks to be measured in flare plume experiments shown in isolation from minor $\mathrm{H}_{2} \mathrm{O}$ peaks with (b) corresponding temperature-dependent linestrengths.

The selected absorption peaks are advantageous because their respective linestrengths exhibit nearlinear scaling with temperature through most of the range of $300 \mathrm{~K}$ to $800 \mathrm{~K}$. A linear response 
has been shown to result in the most sensitive measurements (Rieker et al., 2009). The selection of wavelengths in the near infrared as opposed to the mid-infrared was made to capitalize on the ability to fiber-couple the light. Fiber-coupling would permit the laser driving systems and reference optics to be located a safe distance from the test flares. Stability of the laser systems is highly sensitive to temperature and requires a thermally-stable enclosure.

\subsection{Soot Detection Using Line-of-Sight Attenuation (LOSA)}

Soot detection was performed with a laser tuned near $1654 \mathrm{~nm}$. Methane was the only gas-phase species in the plume that could absorb the light in this spectral region. The laser was tuned away from the dominant methane absorption transitions so only light attenuation due to soot was observed.

Light attenuation due to soot is caused by both light scattering and light absorption. From the rigorous review of light scattering by fractal aggregates by Sorensen (2001) and following the notation of Johnson et al. (2013) in the absence of in-scattering of skylight, Eq. (2.10) can be used to describe the light transmission measured through a soot plume at some wavelength $\lambda[\mathrm{nm}]$. The transmissivity $\tau_{\text {soot }}[-]$ is given by,

$$
\tau_{\text {soot } \lambda}=\exp \left(-\frac{K_{e_{\lambda}}}{\lambda} \int_{s} f_{v}(s) d s\right)
$$

where $K_{e_{\lambda}}[-]$ is the soot extinction coefficient at some wavelength $\lambda$ and $f_{v}$ is the soot volume fraction (SVF) [-] integrated over the optical path $s$. Equation (2.10) describes how the soot extinction coefficient can be broken down into constituent parts,

$$
K_{e_{\lambda}}=6 \pi\left(1+\rho_{S A_{\lambda}}\right) E\left(m_{\lambda}\right)
$$


where $E\left(m_{\lambda}\right)=-\operatorname{Im}\left(\frac{m^{2}-1}{m^{2}+2}\right)[-]$ is the soot absorption function, $m_{\lambda}=n(\lambda)-i k(\lambda)[-]$ is the complex refractive index made up of real and imaginary indices $n$ and $k[-]$, and $\rho_{S A_{\lambda}}[-]$ is the light scattering-to-absorption ratio of soot. The $\lambda$ subscripts denote parameters with wavelength dependence.

From the soot optical property review of Coderre et al. (2011), most rigorous soot optical property measurements are conducted in/near visible wavelengths with most not exceeding $1064 \mathrm{~nm}$ in the infrared. There are some soot optical property measurements that encompass the selected wavelengths, however they typically only consider the extinction coefficient and not its constituents. Of those studies that investigated soot optical properties between 1400-1700 nm, there is some disagreement on $E\left(m_{\lambda}\right)$ or $K_{e_{\lambda}}$ values. However, this is not a limiting concern for the present work which seeks to measure species correlation between soot and $\mathrm{H}_{2} \mathrm{O}$ rather than absolute volume fraction data. Any bias in assumed soot optical properties will affect absolute SVFs but not the strength of correlation with $\mathrm{H}_{2} \mathrm{O}$ volume fraction. Thus, soot optical properties at $1654 \mathrm{~nm}$, the selected wavelength for soot detection, were assumed as elaborated below.

Based on a study by Migliorini et al. (2011), $\rho_{S A}$ is assumed to be $\sim 0.02$ at both wavelengths. In the NIR, the wavelength becomes larger than the soot aggregates in the plume. Beyond this point, in the so-called Rayleigh regime, the light scattering approaches zero as the soot particle size parameter $x_{p}=\frac{2 \pi R_{g m}}{\lambda}$ becomes much less than unity.

The complex refractive index of soot is estimated by assuming correlations from Chang \& Charalampopoulos (1990). Equations for the real and imaginary parts of the complex refractive index are shown in Eq. (2.11) and (2.12), respectively, 


$$
\begin{aligned}
& n(\lambda)=1.8110+0.1263 \ln \lambda+0.0270 \ln ^{2} \lambda+0.0417 \ln ^{3} \lambda \\
& k(\lambda)=0.5821+0.1213 \ln \lambda+0.2309 \ln ^{2} \lambda-0.0100 \ln ^{3} \lambda
\end{aligned}
$$

The assumed properties above can be rolled up into the soot extinction coefficient from Eq. (2.10). At $1654 \mathrm{~nm}$, the extinction coefficient is therefore assumed to be 4.316 [-]. This value will be used to infer soot volume fractions as it relates to optical transmissivity.

\subsection{Simultaneous TDLAS / LOSA}

Until now, the theoretical development of each measurement technique has been treated separately. However, since soot acts as a blackbody, it will not only attenuate light at the $1654 \mathrm{~nm}$ (soot detection) wavelength but also at the $1428 \mathrm{~nm}\left(\mathrm{H}_{2} \mathrm{O}\right.$ vapour detection) wavelength. With $\rho_{S A}$ and $E(m)$ both being wavelength-dependent, attenuation magnitudes will not be equivalent at the two wavelengths. In the absence of any other spectral absorption or light losses, the transmissivities measured at each wavelength can be decomposed into light loss from the two species in Eq. (2.13) and (2.14),

$$
\begin{gathered}
\tau_{w}(v)=\tau_{H_{2} O}(v) \tau_{\text {soot }_{w}} \\
\tau_{s}(v)=\tau_{\text {soot }_{s}}
\end{gathered}
$$

where the subscripts $w$ and $s$ on the transmissivities $\tau$ denote laser wavelengths for the water $(1428 \mathrm{~nm})$ and soot $(1654 \mathrm{~nm})$ detection. To enable spectral profile-fitting to determine $\mathrm{H}_{2} \mathrm{O}$ volume fractions, the soot attenuation component of $\tau_{w}(v)$ must be removed to isolate $\tau_{\mathrm{H}_{2} \mathrm{O}}(v)$. Since $\tau_{\text {soot }}$ is unknown, it must be estimated from $\tau_{\text {soot }_{s}}$. If the ratio of soot optical properties between the two wavelengths is known, then the spectral absorption of $\mathrm{H}_{2} \mathrm{O}$ vapour can be isolated from the broadband soot attenuation through Eq. (2.15), 


$$
\tau_{H_{2} O}(v)=\tau_{w}(v) \frac{1}{\tau_{\text {soot }_{w}}}
$$

Leveraging Eq. (2.9) and (2.10), $\tau_{\text {soot }_{w}}$ can be approximated from $\tau_{\text {soot }_{s}}$ through Eq. (2.16) or, if $\rho_{S A}$ is assumed to have a negligible change between wavelengths, Eq. (2.17) can be used,

$$
\begin{gathered}
\tau_{\text {soot }_{w}}=\exp \left(\ln \left(\tau_{s}\right) \frac{K_{e_{w}}}{K_{e_{s}}} \frac{\lambda_{s}}{\lambda_{w}}\right) \\
\tau_{\text {soot }_{w}}=\exp \left(\ln \left(\tau_{s}\right) \frac{E\left(m_{w}\right)}{E\left(m_{s}\right)} \frac{\lambda_{s}}{\lambda_{w}}\right)
\end{gathered}
$$

These equations imply that since a value of $K_{e_{S}}$ has been assumed, the ratio between the two extinction coefficients is required to properly isolate the $\mathrm{H}_{2} \mathrm{O}$ absorption signal.

Correctly estimating $\tau_{\text {soot }}$ is critical to obtaining true $\mathrm{H}_{2} \mathrm{O}$ vapour absorption signals. Since there is no agreed upon source for accurate soot extinction coefficients in the current wavelength range, a ratio of the extinction coefficients must be measured. The extinction coefficient measurement was a critical step in the experiment conducted in this thesis. Table 2.1 summarizes various studies that have measured soot extinction coefficients for wavelengths at or near the selected wavelengths. Extinction coefficient ratios (ECRs) are presented in the table that were calculated from the soot extinction coefficient results of each study to reflect the anticipated extinction ratios between the selected wavelengths of this thesis. The table also includes the value measured in this thesis, which is discussed in further detail in Chapter 5. 
Table 2.1: Summary of soot extinction coefficient measurements with extinction coefficient ratios (ECRs) calculated for the wavelengths selected for measurement

\begin{tabular}{|l|l|l|l|l|}
\hline Study & Method & Fuel Source & $\begin{array}{l}\text { Wavelength, } \\
\boldsymbol{\lambda}[\boldsymbol{\mu m}]\end{array}$ & $\begin{array}{l}\text { ECR, } \\
\boldsymbol{K}_{\boldsymbol{e}_{\boldsymbol{w}}} / \boldsymbol{K}_{\boldsymbol{e}_{\boldsymbol{s}}}[-]\end{array}$ \\
\hline Blokh (1988) & Scatt/Extinction & Various & $1-6$ & $1.044^{\mathrm{a}}$ \\
\hline Chang \& Charalampopoulos (1990) & Scatt/Extinction & $\mathrm{C}_{3} \mathrm{H}_{6} / \mathrm{O}_{2}$ & $0.4-30$ & $0.976^{\mathrm{b}}$ \\
\hline Köylü \& Faeth (1994) & Extinction & Various & $0.2-5.2$ & $0.945^{\mathrm{c}}$ \\
\hline Krishnan et al., (2001) & Scatt/Extinction & Various & $0.25-5.2$ & $0.969^{\mathrm{c}}$ \\
\hline Zhu et al. (2002) & Extinction & $\mathrm{C}_{2} \mathrm{H}_{2}, \mathrm{C}_{2} \mathrm{H}_{4}$ & $1.31,1.56$ & $0.912,0.998^{\mathrm{d}}$ \\
\hline Present Study & Extinction & $\mathrm{C}_{2} \mathrm{H}_{4}$ & $\mathbf{1 . 4 2 8 , 1 . 6 5 4}$ & $\mathbf{0 . 9 6 4 \pm 0 . 0 1 3}$ \\
\hline
\end{tabular}

${ }^{\text {a }}$ Determined through quoted complex index of refraction relationship and assuming $\rho_{S A}=0.02$.

${ }^{\mathrm{b}}$ Calculated from $n$ and $k$ relationships of Eq. (2.11) and (2.12) and assuming $\rho_{S A}=0.02$.

${ }^{\mathrm{c}}$ Calculated from interpolation of extinction data.

d Soot OPR between $1565 \mathrm{~nm} \& 1314 \mathrm{~nm}$. 


\section{Chapter 3 Optical Technique Validation Simulations}

\subsection{Overview}

The performance of the optical techniques developed in Chapter 2 had to be assessed prior to lab experiments. Simulations were performed to assess the technique's expected performance under realistic flow conditions and to determine expected measurement error. Large eddy simulation (LES) datasets of methane flares in crossflow obtained from the University of Utah were used to generate theoretical soot attenuation and $\mathrm{H}_{2} \mathrm{O}$ absorption signals that could be analyzed. To add fidelity to the simulation, both signals were corrupted with noise of the magnitude measured in preliminary lab experiments. The performance of the spectral analysis technique was then evaluated based on the ability to reproduce the known path-averaged species volume fractions from the LES simulation data.

\subsection{Simulation Setup}

Large eddy simulation (LES) data of methane flares in crossflow were provided by Prof. Jeremy Thornock of the University of Utah. Example instantaneous snapshots of these simulations are shown in Figure 3.1. Line-of-sight (LOS) datasets through various locations in the simulated flare plume were extracted and used to generate theoretical soot and $\mathrm{H}_{2} \mathrm{O}$ vapour attenuation/absorption signals. LOS datasets of $150 \mathrm{~cm}$ path length were selected since it was the path length designed for in the eventual optical system. A large number of locations in the simulated plume were probed to obtain data with a wide variety of flow characteristics including near-flame high temperature data as well as far-field low temperatures with more dispersed combustion products. 

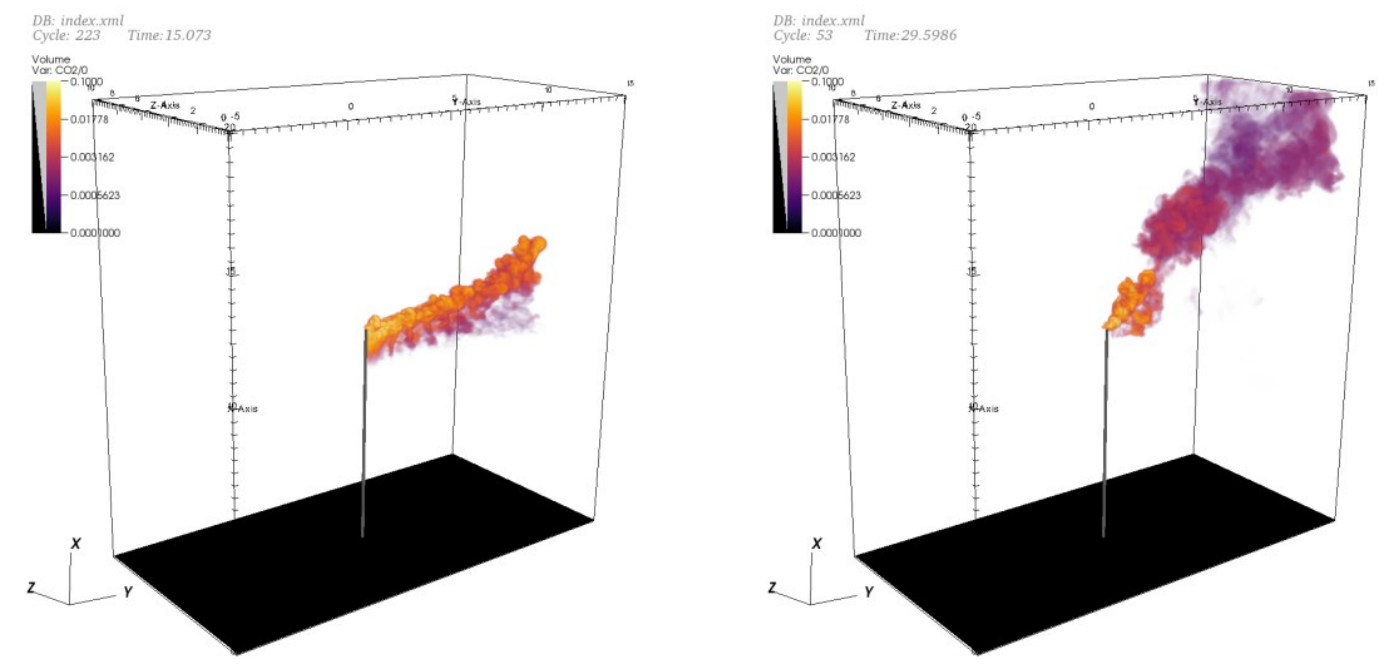

Figure 3.1: Instantaneous visualization of LES methane flare in crossflow (provided by Jeremy Thornock, University of Utah).

The large eddy simulation used a structured mesh with a resolution of $2.5 \mathrm{~cm}$ (i.e., 60 nodes across the $150 \mathrm{~cm}$ path length). Although this spatial resolution is coarse, Appendix A presents a comparison with a more highly resolved dataset and demonstrates similar performance to the results presented in this chapter.

The combustion process in the flare simulation was a single-step reaction that only allowed the combusting methane-air mixtures to produce $\mathrm{CO}_{2}$ and $\mathrm{H}_{2} \mathrm{O}$ vapour; no other pollutants were generated. A nominal soot volume fraction (SVF) field was generated by scaling the combustionderived $\mathrm{CO}_{2}$ volume fraction field by an assumed factor of $1 / 750,000$. From internal findings at the Carleton University Flare Facility (CUFF), this ratio is of similar magnitude to soot/ $\mathrm{CO}_{2}$ volume fraction ratios of a pure propane flare. This value was selected to produce optical attenuation in the range of 0 to $5 \%$. It was not important that the soot field be accurate, only that the distribution be representative of what might be found in a flare to facilitate testing of the optical analysis techniques. 
Theoretical instantaneously measurable signals were generated for each species using the volume fraction and temperature data at each node along the LOS using Eq. (3.1) and (3.2),

$$
\begin{gathered}
\tau_{H_{2} O}(v)=\prod_{k=1}^{k=N} \exp \left(\sum_{i} \frac{q_{k} p}{k T_{k}} \sigma_{v_{c i}}\left(p, T_{k}, q_{k}, v\right) \delta s\right) \\
\tau_{\text {soot }_{\lambda}}=\prod_{k=1}^{k=N} \exp \left(-\frac{6 \pi\left(1+\rho_{S A_{\lambda}}\right) E(m)_{\lambda}}{\lambda} f_{v_{k}} \delta s\right)
\end{gathered}
$$

where $k$ denotes the node position along the LOS up to node $N(60)$, and $\delta s$ is the node width $(2.5 \mathrm{~cm})$ for each absorption transition $i$. Simulated signals for each laser were then generated using Eq. (2.13) and (2.14) to account for the broadband soot attenuation that attenuates light at both wavelengths. Each attenuation signal was also corrupted with normally distributed noise with a standard deviation of $6 \times 10^{-4}[-]$. This value represents apparent attenuation signals caused by laser and detector noise; the value is $50 \%$ higher than the noise observed during initial setup of the optical system and represents a worst case for this analysis.

Since soot optical properties had not been measured, properties had to be assumed from literature. $E(m)$ values were assumed at each wavelength using the relationships described by Chang \& Charalampopoulos (1990) and the soot scattering-to-absorption ratio $\rho_{S A}$ was assumed to be $\sim 0.02$ from Migliorini et al. (2011).

In practice, the Voigt profile is often used to model spectral line broadening since it accounts for homogeneous (natural and collisional) and inhomogeneous (temperature) broadening (Schiff et al., 1994). However, at the selected NIR wavelength of $1428 \mathrm{~nm}$, theoretical inhomogeneous effects contribute absorption of at most $5 \times 10^{-5}[-]$. This is more than an order of magnitude lower 
than the noise at its maximum. For this reason, and the fact that the Voigt profile is much more computationally demanding than the Lorentz profile, the Lorentz profile was used to model line broadening throughout the simulations.

\subsection{Simulation Results}

Individual signals generated from the LOS data from the methane flare simulations were analyzed using the optical techniques developed in Chapter 2. Before any analysis was conducted on the results, values of the spectral fit that returned impossible flow characteristics such as temperatures below ambient or column widths that were above the path length were removed. The individual results that produced column distributions of non-physical parameters typically either had the attenuating noise obscure weak absorption/attenuation signals or represented some distribution of $\mathrm{H}_{2} \mathrm{O}$ along the path that could not be well-described by the column distribution form.

True path-averaged soot volume fraction (SVF) results ranged from 0 to $15 \mathrm{ppb}$ and these were correctly estimated to within $0.02 \mathrm{ppb}$ in all cases. The error in instantaneous path-averaged SVF estimates was solely due to precision error on the simulated light attenuation caused by the corrupting noise. Path-averaged SVF estimates were then used to isolate the $\mathrm{H}_{2} \mathrm{O}$ spectral absorption using Eq. (2.15) and the assumed soot optical properties. This isolated signal was then fitted to a theoretical $\mathrm{H}_{2} \mathrm{O}$ absorption spectrum of a column distribution. Figure 3.2a shows a typical result of the column distribution fit, plotting the column fit spectra over the simulated measured spectra. Figure $3.2 \mathrm{~b}$ and c compare the fitted column profiles of $\mathrm{H}_{2} \mathrm{O}$ volume fraction and temperature with the spatially distributed data along the LOS from the simulation. As indicated in the legend of Figure $3.2 \mathrm{~b}$, the path-averaged volume fraction in this example was 
estimated to within $1.2 \%$ of the true mean. Similarly, the effective temperature was within $2 \mathrm{~K}$ of the path-averaged value along the LOS (Figure 3.2c).
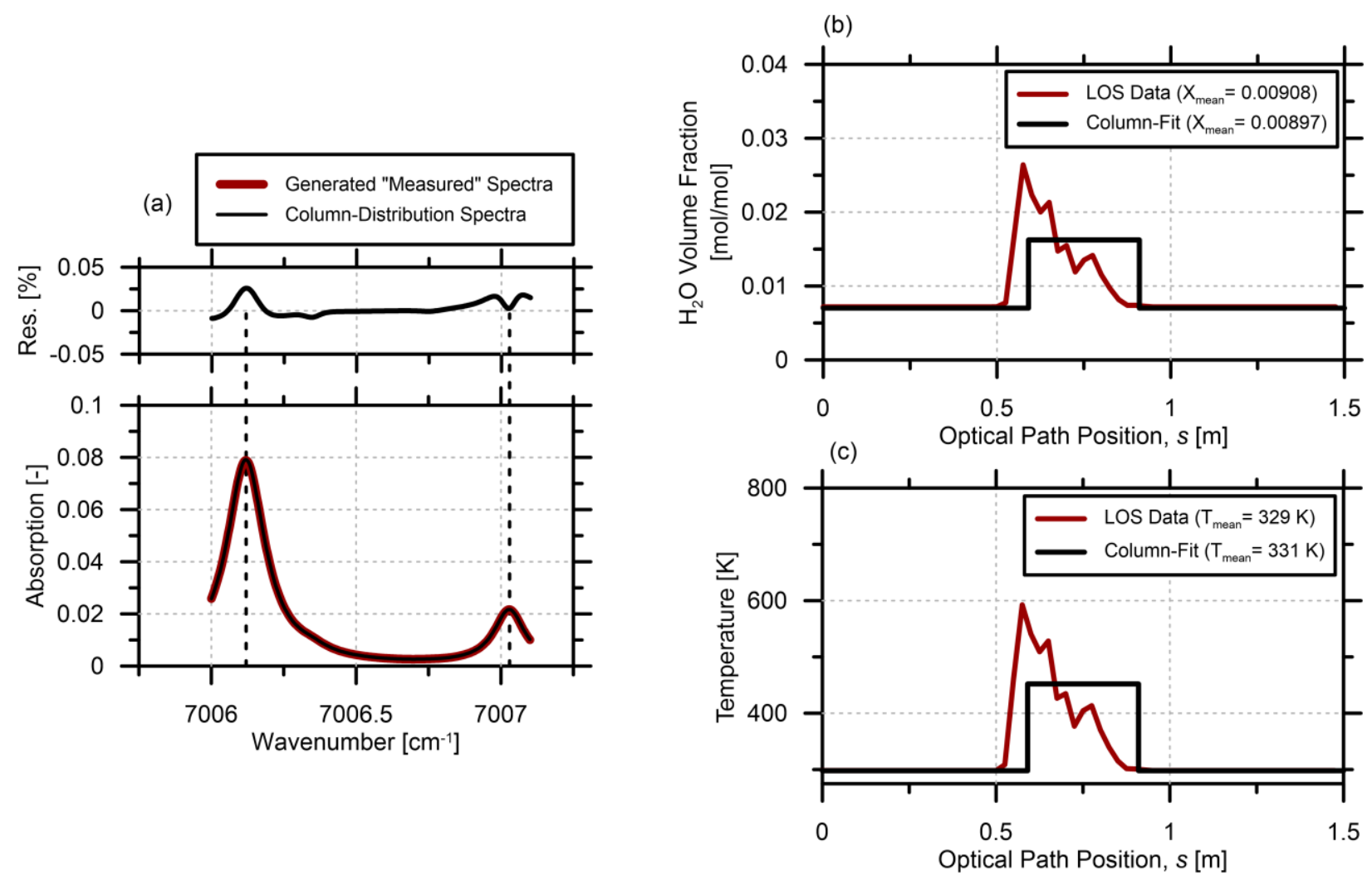

Figure 3.2: Sample profile fitting result where (a) compares the column distribution spectra to the simulated measured spectra, and (b) and (c) compare the column distribution profiles with LOS simulation data for volume fraction and temperature, respectively.

Results for instantaneous snapshots such as those in Figure 3.2 were generated for roughly 9000 LOS datasets to draw more general conclusions about the profile-fitting performance. One important finding was the apparent trend of reduced spectral fitting performance with elevated gas temperature along the LOS.

Figure 3.3 shows the error in path-averaged $\mathrm{H}_{2} \mathrm{O}$ as a function of the peak temperature along each LOS. Above roughly $800 \mathrm{~K}$, the error in $\mathrm{H}_{2} \mathrm{O}$ volume fraction appears to increase significantly. This is likely due to the divergence of the transition linestrengths from near linear 
in the range of 300 to $800 \mathrm{~K}$ as noted in Section 2.1.3. This suggests that placement of the optical path in or just beyond the extent of the flame would adversely impact the accuracy of the system.

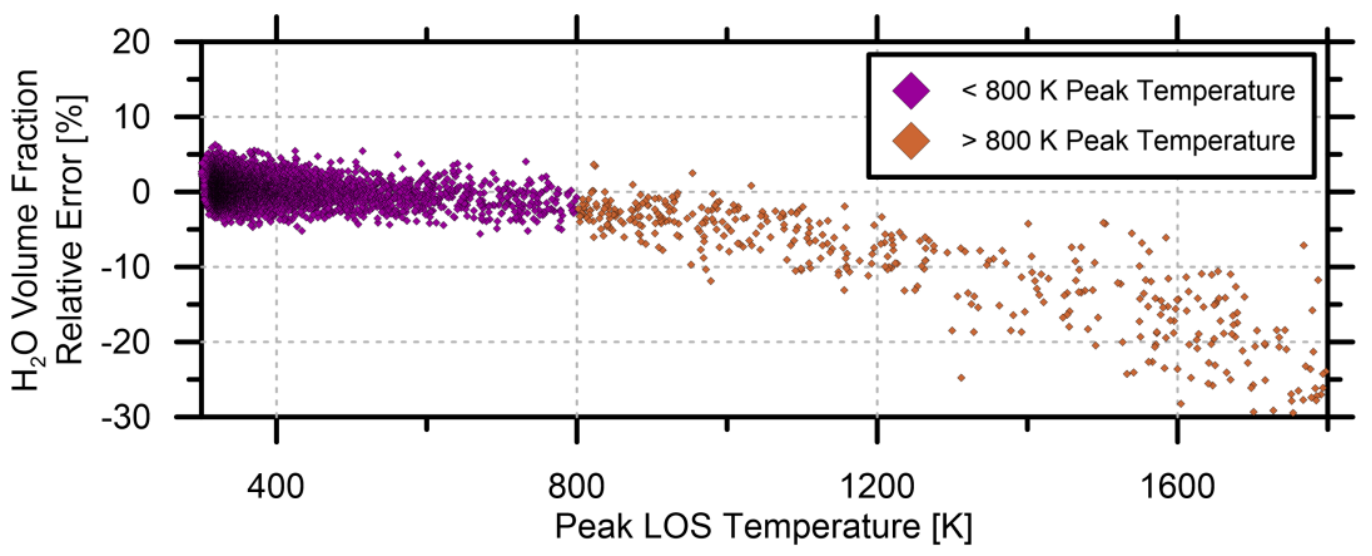

Figure 3.3: Relative error of $\mathrm{H}_{2} \mathrm{O}$ volume fraction results from simulated measured data compared to peak LOS temperature for each result.

According to Poudenx et al. (2004), who used thermocouples to measure temperatures in turbulent jet diffusion flame plumes in crossflow, near-field plume temperatures did not typically exceed $600 \mathrm{~K}$. Their measurements were taken $1.4 \mathrm{~m}$ downstream of a $2.21-\mathrm{cm}$ burner with $2 \mathrm{~m} / \mathrm{s}$ burner exit velocity. In this thesis, the measurements discussed in Chapter 5 used flow velocities that did not exceed $2 \mathrm{~m} / \mathrm{s}$ and took measurements at $2 \mathrm{~m}$ above the burner on buoyancy-driven turbulent flare plumes. Although these two conditions are difficult to compare directly, especially since this thesis presents results from different fuels than Poudenx et al. (2004), these thermocouple measurements provide some indication of expected temperatures. It is therefore conservatively assumed that peak temperatures in the plume will not exceed $800 \mathrm{~K}$ (the apparent performance breakdown in spectral fitting).

Although it is difficult to confirm the validity of this peak temperature assumption in the lab without direct temperature measurements, the simulation results indicate that these high peak temperature results are also distinguishable in the path-averaged temperature estimates. Figure 
3.4a shows that results where peak LOS temperatures were in excess of $800 \mathrm{~K}$ often had pathaveraged temperatures above $320 \mathrm{~K}$; Figure $3.4 \mathrm{~b}$ also indicates that this trend can be discerned from estimated path-averaged temperatures determined from the column distribution results. Estimated path-averaged temperatures for the laboratory measurements will be discussed in Chapter 5 to confirm that path-averaged temperatures are generally less than $320 \mathrm{~K}$.

(a)

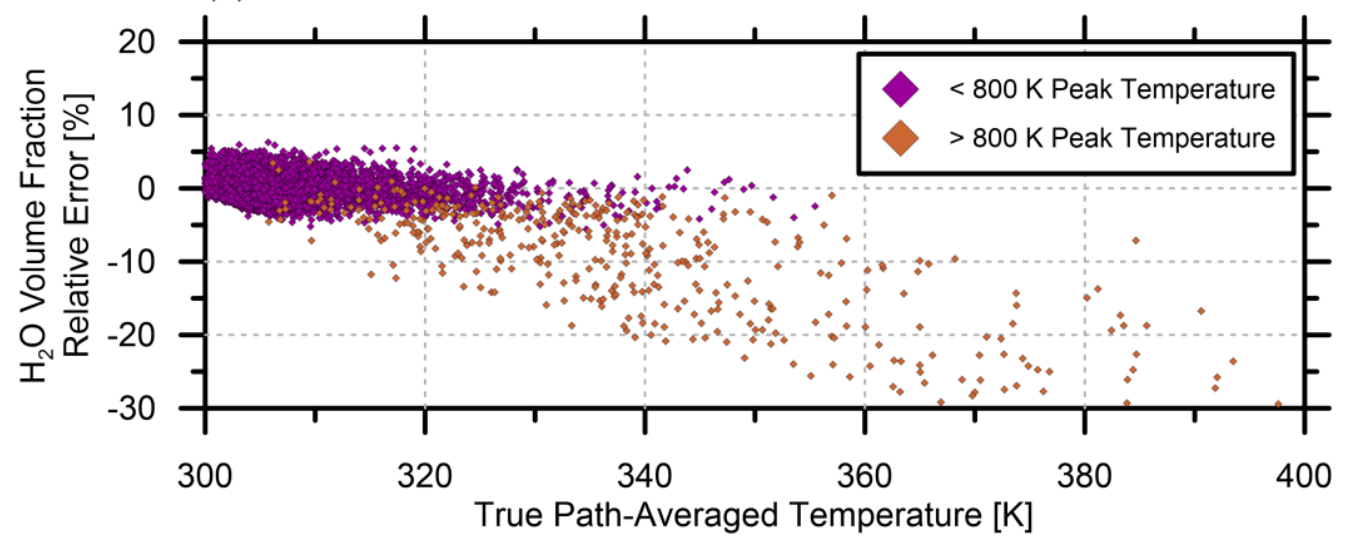

(b)

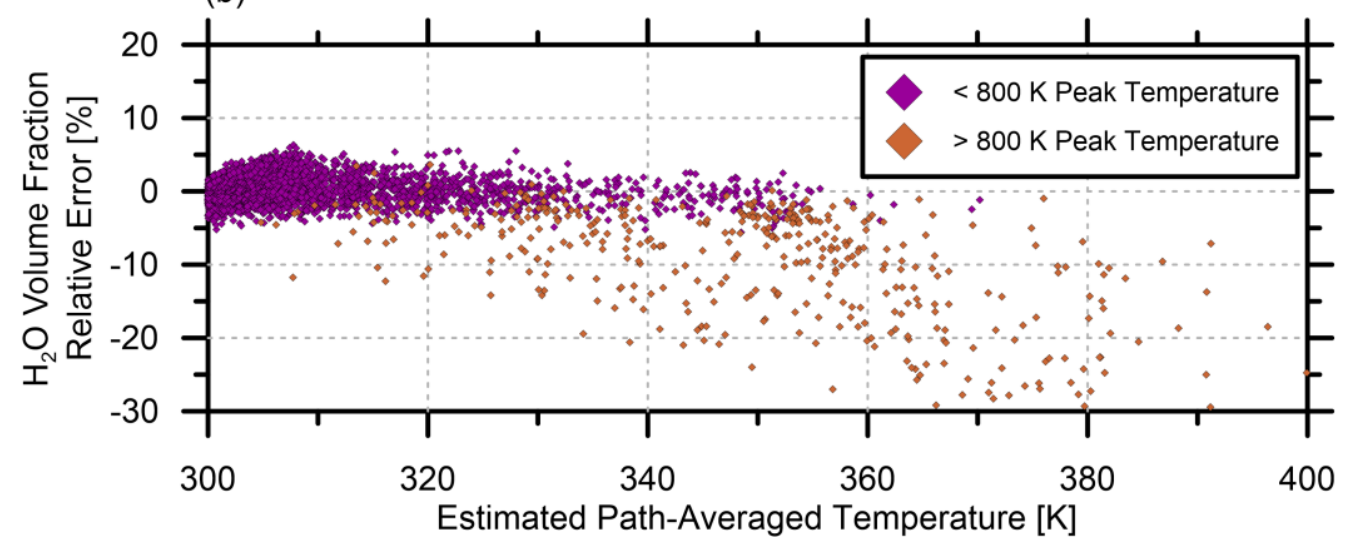

Figure 3.4: Relative error of path-averaged $\mathrm{H}_{2} \mathrm{O}$ compared to (a) true path-averaged temperatures and (b) estimated path-averaged temperatures calculated from column distribution results.

The performance of the spectral fit using column distributions was also assessed in terms of the sum-of-squared-errors (SSE) that is returned by the Nelder-Mead algorithm. The SSE is a singlevalued residual that indicates the closeness of fit between the measured spectra and the column distribution theoretical spectra. Figure 3.5 shows the trend of increased error in $\mathrm{H}_{2} \mathrm{O}$ estimates 
with increasing SSE. The figure implies that the SSE may be used as a metric to determine the accuracy of the individual results. Generally, however, if peak LOS temperatures are assumed to not exceed $800 \mathrm{~K}$ then the path-averaged $\mathrm{H}_{2} \mathrm{O}$ volume fraction is typically estimated to within $5 \%$. For data in Figure 3.5 with peak temperatures below $800 \mathrm{~K}$, the relative error was $-0.25 \%$ on average with $95 \%$ of measurements being within $\pm 6.74 \%$.

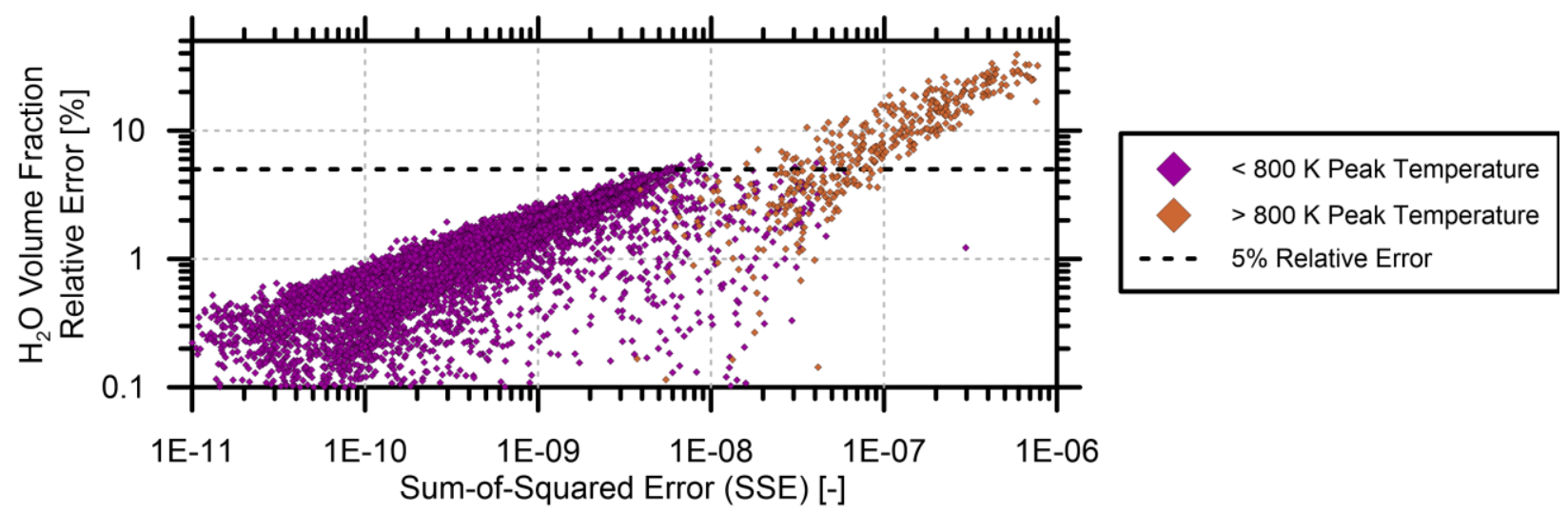

Figure 3.5: Trend of increased relative error of $\mathrm{H}_{2} \mathrm{O}$ volume fractions with increase in SSE from the Nelder-Mead optimization search

Results of soot and $\mathrm{H}_{2} \mathrm{O}$ volume fractions can be inspected in a scatter plot for correlation. Figure 3.6 shows scatter plots for the integrated species volume fractions (a) with and (b) without results where peak temperatures do not exceed $800 \mathrm{~K}$. There is a strong correlation between species which is expected in this simulation because the soot and $\mathrm{H}_{2} \mathrm{O}$ are correlated by design.

The correlation results were also interpreted by isolating the combustion-derived $\mathrm{H}_{2} \mathrm{O}$ estimates from the column distribution and computing the soot-to- $\mathrm{H}_{2} \mathrm{O}$ volume fraction ratio. A histogram of the apparent soot- $\mathrm{H}_{2} \mathrm{O}$ ratio after the removal of results where peak temperatures exceed $800 \mathrm{~K}$ is shown in Figure 3.7. The histogram indicates good agreement of the mean soot$\mathrm{H}_{2} \mathrm{O}$ ratio however the distribution of estimates has a positive skewness. This skewness is likely an artefact of the $\mathrm{H}_{2} \mathrm{O}$ estimates being slightly underestimated. The finding of inherent skewness 
in the simulation will be an important point of comparison to the measurement results. If there exists short-lived burst of high soot production uncorrelated with $\mathrm{H}_{2} \mathrm{O}$, the expected result would be a soot- $\mathrm{H}_{2} \mathrm{O}$ ratio of positive skewness; this was previously discussed in Chapter 1 . If this positive skewness of soot- $\mathrm{H}_{2} \mathrm{O}$ ratios exists in the flare plumes, it must be of a higher skewness than the result presented in Figure 3.7 to be unambiguously identified.

(a)

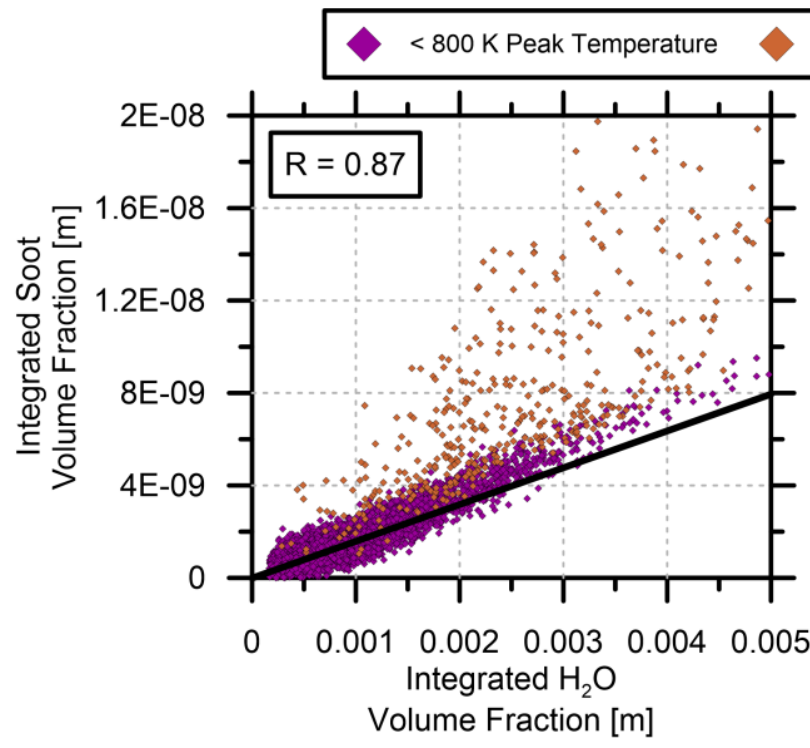

(b)

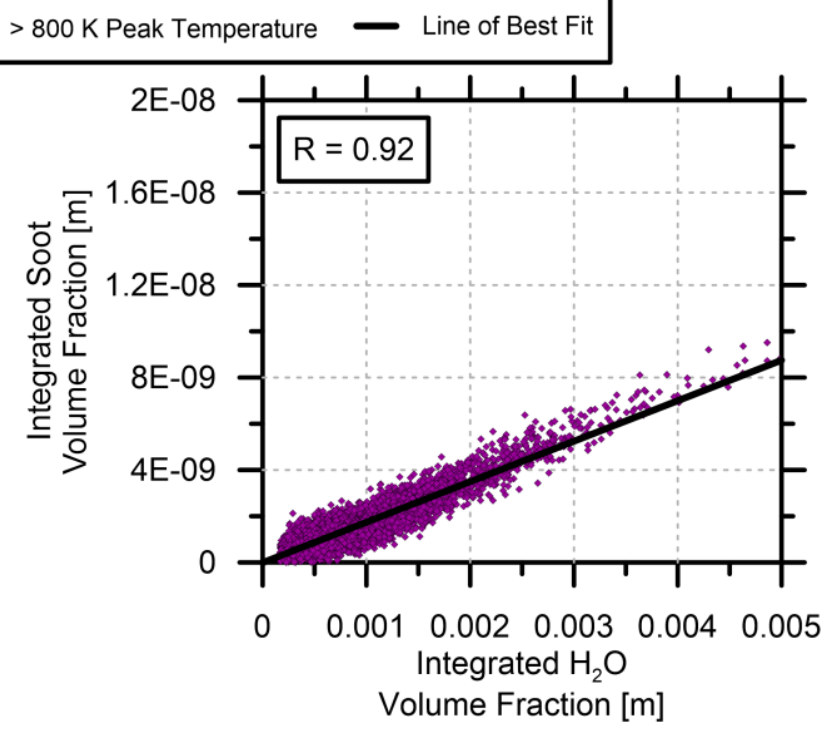

Figure 3.6: Scatter plots of soot and combustion-derived $\mathrm{H}_{2} \mathrm{O}$ volume fractions (a) before and (b) after the removal of results where peak line-of-sight temperatures exceed $800 \mathrm{~K}$. 


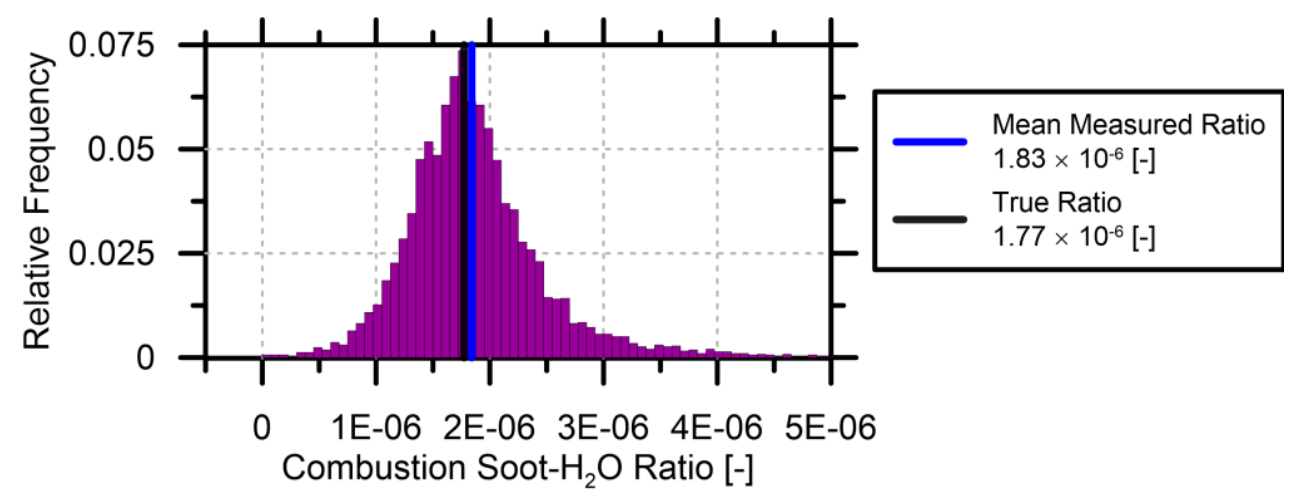

Figure 3.7: Histogram of soot to combustion-derived $\mathrm{H}_{2} \mathrm{O}$ volume fraction ratio. The simulation results show a standard deviation of $6.92 \times 10^{-7}$, skewness of 1.08 and good agreement between mean values.

Imposing some cut-off sum-of-squared-error (SSE) value was considered to remove spectral fit results that were deemed insufficient to yield accurate $\mathrm{H}_{2} \mathrm{O}$ estimates. The effect of such a cut-off was tested by computing relevant statistics of the soot- $\mathrm{H}_{2} \mathrm{O}$ results with different cut-off SSE values. The resulting statistics are shown in Table 3.1. As the cut-off SSE became stricter, the mean soot- $\mathrm{H}_{2} \mathrm{O}$ ratio was more accurately approximated, the standard deviation of the ratio reduced, and the apparent skewness of the distribution was also reduced. This suggests that if some inherent skewness of the true soot- $\mathrm{H}_{2} \mathrm{O}$ ratios exist in the lab experiments, increasingly strict SSE cut-off values should decrease apparent skewness resulting from the measurement itself and leave only the skewness of the soot $-\mathrm{H}_{2} \mathrm{O}$ ratio. The effect of SSE cut-off selection is discussed in Appendix D in reference to measurements presented in Chapter 5.

Table 3.1: Soot- $\mathrm{H}_{2} \mathrm{O}$ ratio statistics and correlation coefficient for different SSE cut-off values

\begin{tabular}{|c|c|c|c|c|c|}
\hline SSE Cutoff & $\begin{array}{c}\text { Results } \\
\text { Removed (\%) }\end{array}$ & $\begin{array}{c}\text { Mean Soot- } \mathrm{H}_{2} \mathrm{O} \\
\text { Ratio }\left(\times 10^{-6}\right)\end{array}$ & $\begin{array}{l}\text { Std. Deviation } \\
\text { of ratio }\left(\times 10^{-7}\right)\end{array}$ & $\begin{array}{c}\text { Ratio } \\
\text { Skewness }\end{array}$ & $\begin{array}{c}\text { Correlation } \\
\text { Coeff. (R) }\end{array}$ \\
\hline None & 0 & $1.83 \mathrm{E}-06$ & 6.92 & 1.08 & 0.92 \\
\hline $1 \times 10^{-8}$ & 2 & $1.83 \mathrm{E}-06$ & 6.98 & 1.10 & 0.90 \\
\hline $5 \times 10^{-9}$ & 4 & $1.82 \mathrm{E}-06$ & 6.89 & 1.04 & 0.90 \\
\hline $1 \times 10^{-9}$ & 29 & $1.80 \mathrm{E}-06$ & 5.16 & 0.90 & 0.93 \\
\hline $5 \times 10^{-10}$ & 38 & $1.77 \mathrm{E}-06$ & 4.31 & 0.69 & 0.95 \\
\hline $1 \times 10^{-10}$ & 81 & $1.75 \mathrm{E}-06$ & 3.54 & 0.24 & 0.97 \\
\hline $5 \times 10^{-11}$ & 92 & $1.72 \mathrm{E}-06$ & 3.49 & 0.26 & 0.97 \\
\hline
\end{tabular}


One consideration that has yet to be discussed but was considered as a possible cause of measurement error was the poor estimation/measurement of ambient $\mathrm{H}_{2} \mathrm{O}$ volume fraction which was a necessary input for the column distribution fitting. To assess the potential impact of poor ambient $\mathrm{H}_{2} \mathrm{O}$ inputs, LOS datasets were reprocessed with intentionally-biased $\mathrm{H}_{2} \mathrm{O}$ estimates that varied by up to $25 \%$ relative error from the true value. The results of one such fit is shown in Figure 3.8. In this example, despite the absolute characteristics of each column fit having changed, the relative error in path-averaged results did not increase by more than $1 \%$. Here, the worst column fit was caused by the ambient $\mathrm{H}_{2} \mathrm{O}$ input that was biased low by $25 \%$ and yielded a $2 \%$ relative error. In general, errors in path-averaged $\mathrm{H}_{2} \mathrm{O}$ volume fractions were not significantly increased by poor estimates of ambient $\mathrm{H}_{2} \mathrm{O}$ volume fraction and the column distribution fit appears to be self-compensating. This intentional biasing of ambient $\mathrm{H}_{2} \mathrm{O}$ volume fractions represents a worst case as the ambient $\mathrm{H}_{2} \mathrm{O}$ was measured in between laboratory tests and did not have a relative change of more than $8 \%$. 
(a)

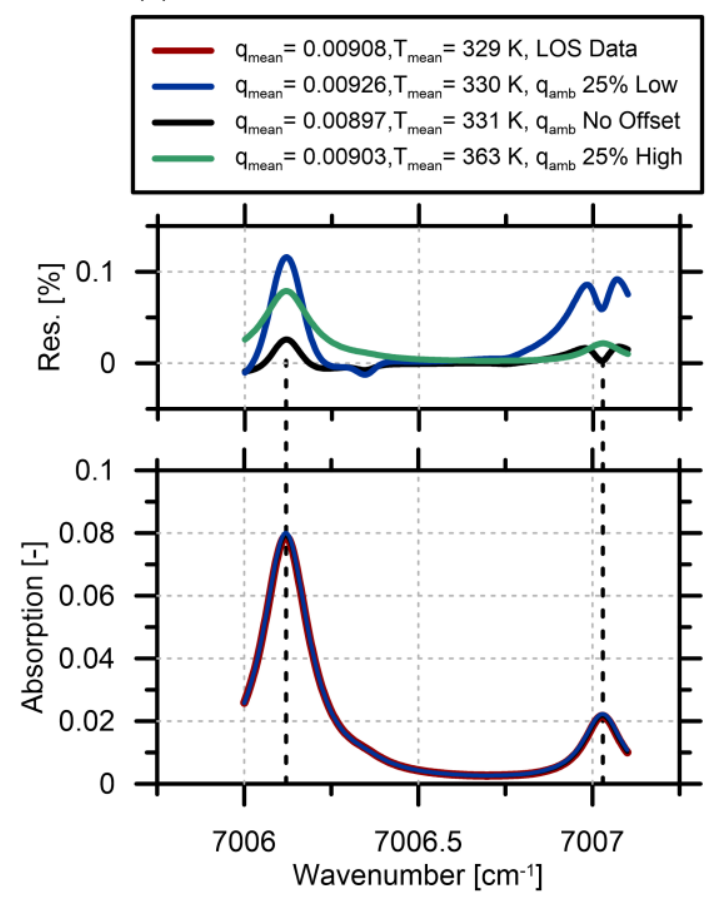

(b)

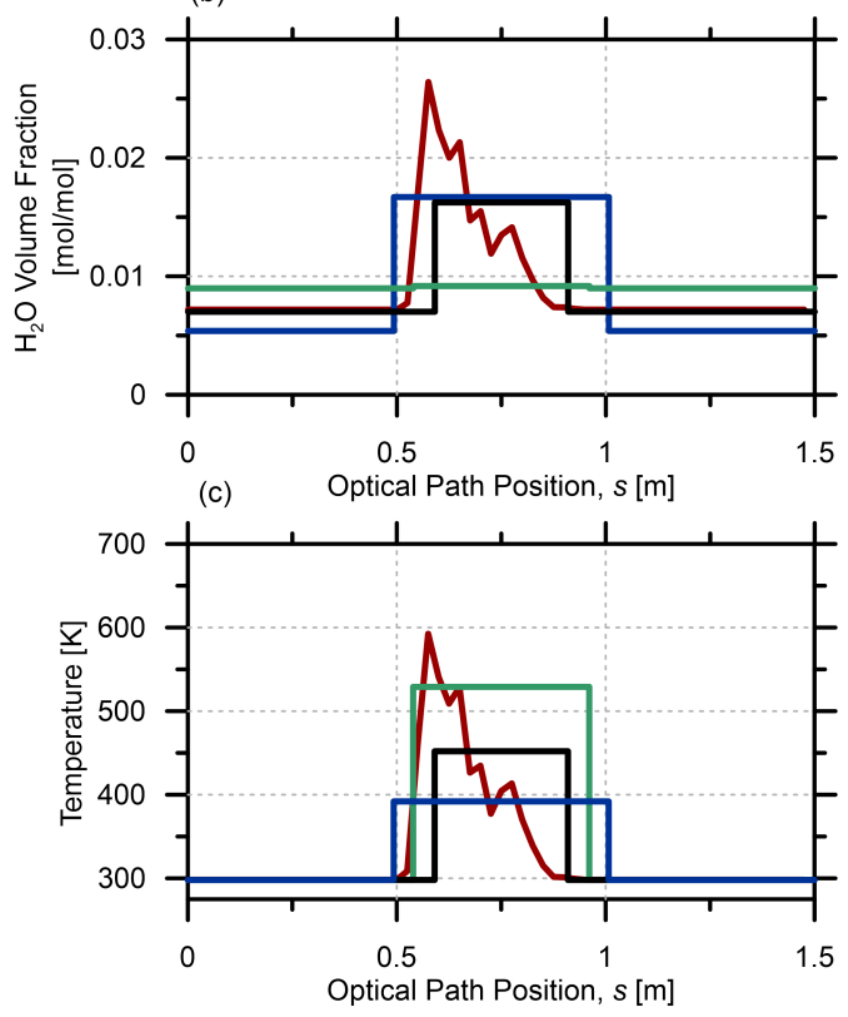

Figure 3.8: Sample of (a) spectral profile fitting results with intentionally-biased ambient $\mathrm{H}_{2} \mathrm{O}$ inputs with (b) and (c) showing the spatially distributed data against column fit results.

Overall, the optical techniques appeared to perform well in simulation. Soot volume fraction estimates were limited by simulated instrument noise, while the spectral profile-fitting algorithm performed well so long as peak temperatures along the line-of-sight were below roughly $800 \mathrm{~K}$. Based on measurements by Poudenx et al. (2004), expected plume temperatures would not likely exceed $500 \mathrm{~K}$. To check this in the experiments, path-averaged temperature estimates from flare measurements in Chapter 5 were compared with path-averaged temperatures of Figure 3.4. The simulation results also indicated that the path-averaged $\mathrm{H}_{2} \mathrm{O}$ volume fractions are not highly sensitive to ambient $\mathrm{H}_{2} \mathrm{O}$ estimates. However, ambient $\mathrm{H}_{2} \mathrm{O}$ measurements were still taken periodically throughout the experimental campaign. 


\section{Chapter 4 Experimental Setup}

\subsection{Overview}

This chapter discusses the design of the optical system (laser driving systems, launch and collection optics, reference signals, etc.), validation of the optical methods through controlled $\mathrm{H}_{2} \mathrm{O}$ vapour experiments, and the flare facility itself. The designed optical system was capable of making simultaneous path-averaged measurements of soot and $\mathrm{H}_{2} \mathrm{O}$ volume fractions at a maximum rate of $200 \mathrm{~Hz}$ limited by the $1428 \mathrm{~nm}\left(\mathrm{H}_{2} \mathrm{O}\right)$ laser. The launch and collection optics were mounted to a three-axis traverse to allow for accurate positioning of the optics above the flare. Lab-scale flares were generated from custom gas mixtures at the Carleton University Flare Facility (CUFF). The laser driving systems and reference optics were located away from the flare to avoid detrimental effects from heating. All detector signals were streamed to a remote acquisition system at a rate of $1 \mathrm{MHz}$ per channel.

\subsection{Laser Diode \& Laser Driving Systems}

The optical system used two distributed feedback (DFB) lasers at nominal wavelengths of $1428 \mathrm{~nm}$ and $1654 \mathrm{~nm}$ for $\mathrm{H}_{2} \mathrm{O}$ vapour and soot detection respectively. DFB lasers are $p-n$ semiconductor diode lasers where the spectrally-narrow emitted radiation can be used to measure individual absorption peaks of $\mathrm{H}_{2} \mathrm{O}$ or held at a constant wavenumber away from absorption peaks to measure broadband light attenuation due to soot. Lasing is achieved by applying a current across the $p-n$ diode junctions. The current causes electron-hole recombination which can emit recombinational energy in the form of electromagnetic radiation. The spectral linewidth of emitted light is generally on the order of several $\mathrm{cm}^{-1}$ (Demtröder, 2014). The radiative emission is amplified by multiple reflections off the end-surfaces in the diode. One of the end surfaces is a diffraction grating which 
only permits the transmission of a spectrally-narrow wavelength (D'Amato, 2006). The DFB lasers used in this system had linewidths at least 3 orders of magnitude more narrow than the full width of the absorption peaks to be inspected.

The laser output wavelength may be tuned by changing the diode package's temperature. The change in diode temperature alters the pitch of the diffraction grating through thermal expansion and allows the output to be scanned across a narrow spectral region (typically $2 \mathrm{~nm}$ or $9.8 \mathrm{~cm}^{-1}$ for the $1428 \mathrm{~nm}$ diode) (Saleh $\&$ Teich, 2007). In the DFB lasers supplied by NTT Electronics a thermoelectric cooler (TEC) unit can be used to modify diode temperature. Temperature-tuning may be used to coarsely position the diode's output over a desired spectral region; however, this process is relatively slow compared to expected timescales in the flare plumes. For rapid tuning during experiments, the laser injection current can be swept at a high rate to rapidly and finely tune the diode output wavenumber. Current-tuning has the same effect of pitch-shifting the diffraction grating but on a much shorter timescale. The nominal $1428 \mathrm{~nm}$ (7002.8 $\mathrm{cm}^{-1}$ ) DFB diode used to measure $\mathrm{H}_{2} \mathrm{O}$ was able to scan across the absorption peaks at a rate of $200 \mathrm{~Hz}$ as discussed in Section 2.1.2. The purchased diodes were hermetically sealed in 14-pin butterfly style packages and the emitted radiation from each diode was coupled into $9-\mu \mathrm{m}$ core, $125-\mu \mathrm{m}$ cladding fiber optic cables with fiber-coupled angled physical contact (FC/APC) connectors. Each 14-pin butterfly package was housed in an ILX laser mount.

The laser diodes were controlled using Stanford Research Systems (SRS) laser diode controllers (LDCs). Each LDC maintained the set TEC temperature of the diode and could maintain or sweep the diode injection current. Custom injection current sweep patterns were created by sending the LDC a voltage waveform that was converted by the LDC to a current waveform sweep. Input waveforms were generated externally as discussed in Section 4.5. 


\subsection{Photodiode Detectors}

Indium Gallium Arsenide (InGaAs) $p-n$ semiconductor photodiodes from Thorlabs Inc. (PDA20CS) were used to detect optical intensity at various locations within the system. The Thorlabs detectors had a 2-mm diameter detector area and responded to $800-1700 \mathrm{~nm}$ radiation. The detectors produced a current proportional to the input optical power (Saleh \& Teich, 2007) and had switchable gain transimpedance amplifiers to output 0-5 V signals. The proportionality between input optical power and diode output current, referred to as the responsivity, is generally wavelength-dependent. The published responsivity curve for the PDA20CS detector elements is shown in Figure 4.1. The responsivities were roughly $0.97 \mathrm{~A} / \mathrm{W}$ at the chosen measurement wavelengths of the current experiment, identified with black dots.

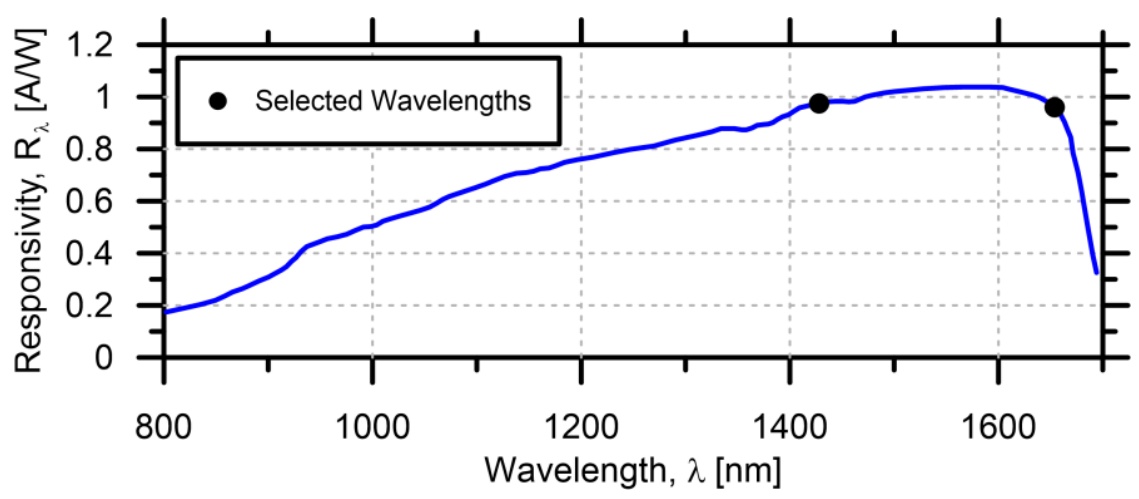

Figure 4.1: Typical responsivity of PDA20CS Detectors. Adapted from Thorlabs (2017).

The voltage output $V_{\text {out }}[\mathrm{V}]$ from the detector can be described by Eq. (4.1),

$$
V_{\text {out }}=R_{\lambda} A P\left(\frac{R_{\text {load }}}{R_{\text {load }}+R_{S}}\right)+V_{D C}
$$

where $R_{\lambda}[\mathrm{A} / \mathrm{W}]$ is the diode responsivity, $A[-]$ is the detector gain setting, $P[\mathrm{~W}]$ is the incident optical power, and $R_{S}$ and $R_{\text {load }}$ are the series and load resistors, each nominally $50 \Omega$ resistance. The term $V_{D C}[\mathrm{~V}]$ is referred to as dark current voltage and represents the intrinsic current leakage 
through the biased photodiode. In practice, ratios of optical intensity are necessary to determine transmissivity or absorption. To accomplish this, the dark current voltage is measured in advance of an experiment and subtracted from each detector signal.

\subsection{Optical System}

\subsubsection{Reference \& Launch Optics}

Fiber-coupled light from each diode was sent through separate fiber-optic reference networks which tapped off fractions of the light for analysis in reference optics. The remaining light from each network was then coupled into a single fiber and sent to an open path beam collimator that launched the light through the test medium. Figure 4.2 shows a schematic of the reference and launch optics; major components are summarized in Table 4.1. All fiber-based components used 9- $\mu \mathrm{m}$ core, $125-\mu \mathrm{m}$ cladding fibers to satisfy the V-parameter condition for single-mode transmission, in which $V<2.405$ where $V=2 \pi \frac{a}{\lambda} N A$ and $a$ is the fiber core radius [nm], $\lambda[\mathrm{nm}]$ is the operating wavelength, and $N A[-]$ is the numerical aperture of the fiber (Saleh \& Teich, 2007). FC/APC connectors were used at the ends of the single-mode fibers (SMFs) to minimize back reflections throughout the system.

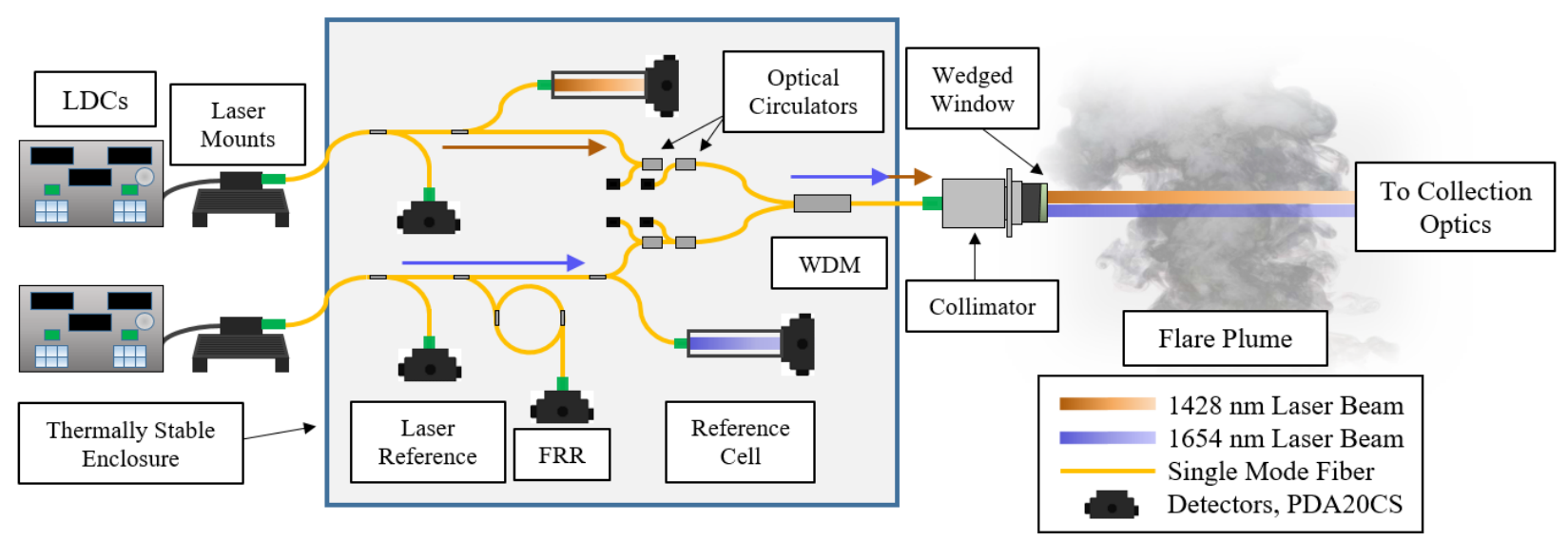

Figure 4.2: Schematic of the laser driving systems and reference and launch optics 
Each reference network was comprised of a laser reference, a fiber-ring resonator (FRR), and a reference cell connected via optical taps from the main transmission fiber. The light in the main fibers was passed through optical circulators to prevent light back-reflections in the wavelength division multiplexer (WDM) which combined the two beams of light into a single fiber. The combined light was then sent through armoured fiber optic cables to the launch collimator located near the test flare. The end of the launch collimator was fitted with a wedged window to prevent optical back-reflections in the open path between launch and collection optics. The major components from Table 4.1 are described in the subsections that follow.

Table 4.1: Major Components of Laser Driving Systems, Reference and Launch Optics

\begin{tabular}{|l|l|l|l|}
\hline Component & Part No. & Provider & Notes/Description \\
\hline Collimator & RC02APC-P01 & Thorlabs & $\begin{array}{l}\text { Protected Silver Reflective Collimator, } 450 \mathrm{~nm}-20 \mu \mathrm{m}, \emptyset 2 \mathrm{~mm} \\
\text { Beam, FC/APC }\end{array}$ \\
\hline Wedged Window & WW10530-C & Thorlabs & $\emptyset 1 / 2 "$ Wedged N-BK7 Window, AR Coating: $1050-1700 \mathrm{~nm}$ \\
\hline Optomechanics & Assorted & Thorlabs & Assorted lens tubes, kinematic mounts, posts etc. \\
\hline $\begin{array}{l}\text { WDM (Wavelength } \\
\text { Division Multiplexer) }\end{array}$ & $\begin{array}{l}\text { WDM-12N-111- } \\
1428 / 1650\end{array}$ & Oz Optics & WDM @ 1428/1650 nm, 60 dB return loss, 9/125 $\mu \mathrm{m}$ fiber \\
\hline Circulator & $6015-3-A P C$ & Thorlabs & Fiber Optic Circulator, 1525 - 1610 nm, SMF, FC/APC \\
\hline Reference Cell & $\begin{array}{l}\text { FC-T(25x20)- } \\
\text { NA-MgF2 }\end{array}$ & $\begin{array}{l}\text { Wavelength } \\
\text { References }\end{array}$ & $\begin{array}{l}\text { 25mm OD glass tube, 20cm path, gas inlet and outlet ports. } \\
\text { Fritted mag. fluoride windows. Windows are wedged and tilted. }\end{array}$ \\
\hline Detectors & PDA20CS & Thorlabs & $\begin{array}{l}\text { InGaAs Switchable Gain Amplified Detector, } 800-1700 \mathrm{~nm}, 10 \\
\text { MHz BW, 3.14 mm² Open Area }\end{array}$ \\
\hline Fiber Components & Various & Various & Fiber cables, 95:5 and 50:50 splitters, bulkhead connectors, etc. \\
\hline Laser Diodes & $\begin{array}{l}\text { NLK1E5GAAA, } \\
\text { NLK1U5FAAA }\end{array}$ & $\begin{array}{l}\text { NTT } \\
\text { Electronics }\end{array}$ & $\begin{array}{l}\text { For 1428 nm and 1654 nm, respectively. 20 and } 15 \mathrm{~mW} \text { output } \\
\text { power; 14-pin Butterfly packaging }\end{array}$ \\
\hline Laser Mount & LDM-4984 & Newport & Laser Diode Mount, 14-pin Butterfly style \\
\hline $\begin{array}{l}\text { LDC (Laser Diode } \\
\text { Controller) }\end{array}$ & LDC500 & SRS & LDC500 Series - LD driver \& TEC controller \\
\hline
\end{tabular}

\section{Laser Reference}

Since the laser diode controller (LDC) and diode do not indicate laser output power, a portion of the light was sent directly to a laser reference detector to monitor output optical power of each laser. Laser intensity drift is most prevalent in the early stages of the diode's life. Laser diode manufacturers subject new diodes to wear-in tests to limit the intensity drift during its intended 
operation (Johnson, 2006). However, intensity drift may still occur and must be monitored. An initial laser reference signal was acquired prior to each test and monitored for fluctuations during the experiment.

\section{Reference Cell}

Fiber-coupled gas reference cells of known path length and gas composition were interrogated by the light entering the reference cell arm of each fiber network. For the $1654 \mathrm{~nm}$ laser network, a 16.5-cm path length reference cell filled with $5 \%$ methane by volume (balance of $\mathrm{N}_{2}$ ) was used. For the $1428 \mathrm{~nm}$ laser, an 80 -cm path length, $2.16 \% \mathrm{H}_{2} \mathrm{O}$ vapour (balance $\mathrm{N}_{2}$ ) reference cell was used. Both reference cells were provided by Wavelength References. The spectral absorption signal seen from the reference cell allowed for drift-free absolute wavelength references at each wavecenter by comparing peak locations to the HITRAN database. The full spectral range of each laser is shown in Figure 4.3 and Figure 4.4 with absorption spectra observed in the gas reference cells.

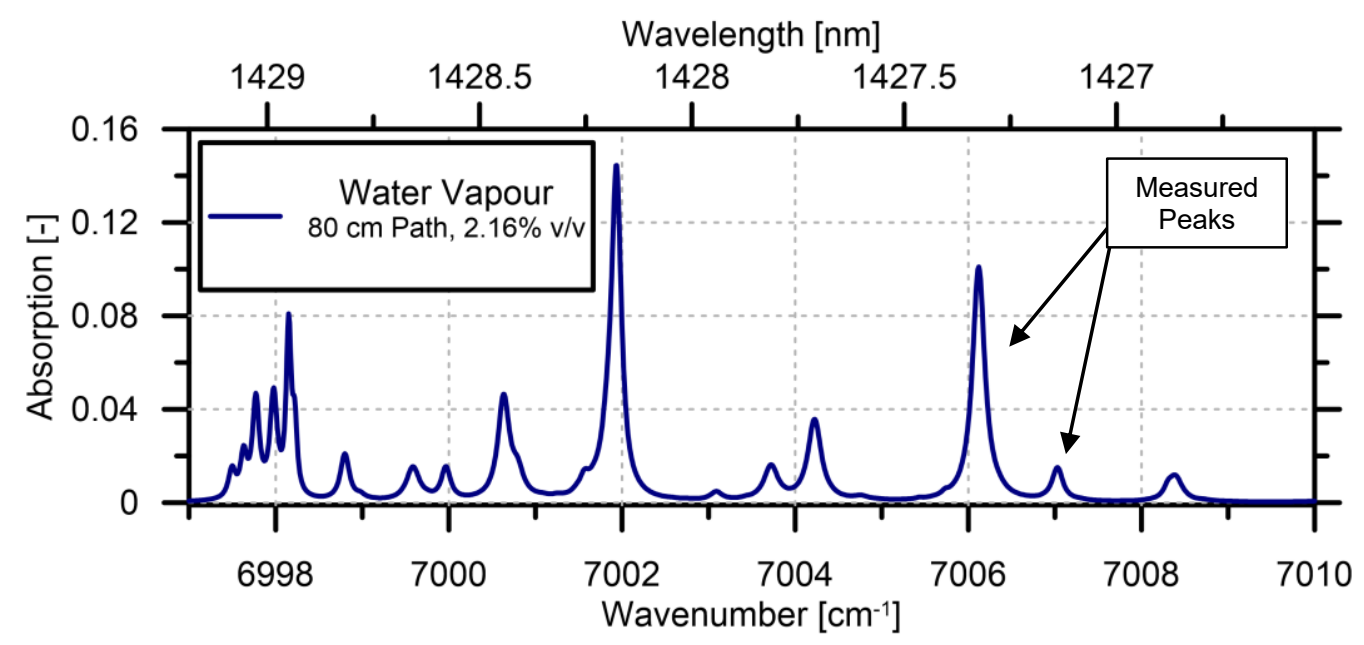

Figure 4.3: $\mathrm{H}_{2} \mathrm{O}$ absorption spectra observed in the $\mathrm{H}_{2} \mathrm{O}$ reference cell with the $1428 \mathrm{~nm}$ laser. The chosen peaks for measurement are noted in the region of $7006-7007 \mathrm{~cm}^{-1}$. 


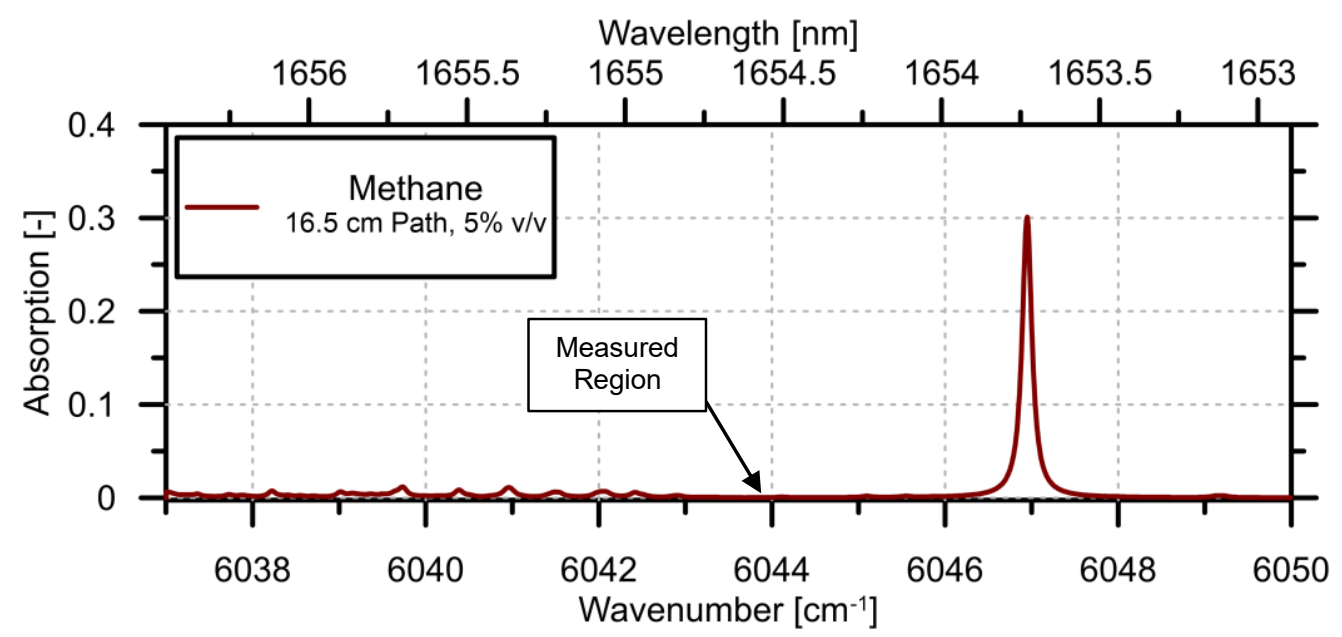

Figure 4.4: Methane absorption spectra observed in the reference cell for the $1654 \mathrm{~nm}$ laser. The measured wavenumber location was selected to avoid any light absorption from methane.

\section{Fiber-Ring Resonator}

Although the reference cells provided absolute wavenumber reference points at absorption peaks, they could not provide references during laser current-tuning since the rate of change of the wavenumber during the scan was not known. To assign wavenumbers to the absorption signals during the current-tuning scan, the reference cell was used in conjunction with a fiber-ring resonator (FRR). The FRR is an optical feedback component where light is forced to interact with itself as it is sent through a ring of fiber. The constructive/destructive interference of light produces an optical fringing pattern which has a predictable fringe spacing. The optical frequency shift between any two neighbouring fringes, called the free spectral range (FSR) denoted as $\Delta v[\mathrm{~Hz}]$, is described by Eq. (4.2),

$$
\Delta v=\frac{c}{n d}
$$

where $c\left[3 \times 10^{8} \mathrm{~m} / \mathrm{s}\right]$ is the speed of light in a vacuum, $d[\mathrm{~m}]$ is the physical length of the fiber in the ring [m], and $n$ [-] is the index of refraction of the travel medium (i.e., fiber optic cable) (Saleh \& Teich, 2007; Urquhart, 1987). 
Since $n$ and $d$ were not sufficiently well-known, the FSR was determined by scanning the laser across multiple absorption peaks of known spectral locations and observing the number of optical fringes between the peaks. The FRR used in this system was characterized using a laser at $1358 \mathrm{~nm}$ and yielded an FSR of $\sim 0.345 \mathrm{GHz}\left(0.0115 \mathrm{~cm}^{-1}\right)$. During experiments where the $1428 \mathrm{~nm}$ laser was being current-tuned, the absorption peak wavecenter $v_{c}$ was determined from the reference cell signal. The FRR peaks were then used to assign wavenumber values to the rest of the signal. Figure 4.5 shows a sample scan over the two absorption peaks of the reference cell with the FRR signal overlaid.
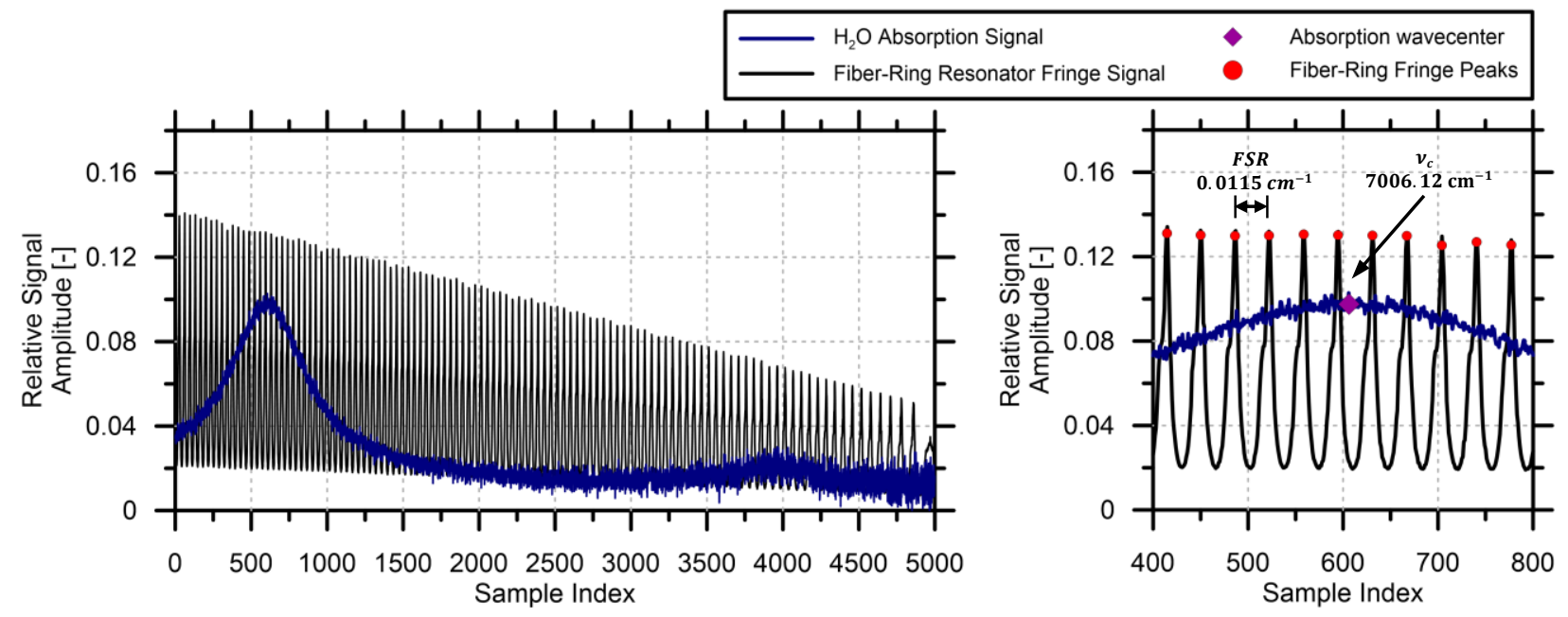

Figure 4.5: $\mathrm{H}_{2} \mathrm{O}$ absorption observed in reference cell overlaid with the FRR signal.

Wavelength-Division Multiplexing

The light from each laser exiting the fiber networks was combined into a single fiber prior to being sent to the launch collimator. Although a simple beam-combiner could accomplish this task, this approach would be susceptible to significant intensity losses. Instead, a custom-made wavelengthdivision multiplexer (WDM) was used to combine the beams while minimizing intensity losses. 


\section{Optical Circulators}

Although the WDM reduces intensity losses of the beams being combined, non-negligible crossreflections were measured. Cross-reflections refer to light from one fiber network being allowed into the opposing fiber network, which can have undesirable, even detrimental effects on the diode that receives this optical feedback (Tkach \& Chraplyvy, 1986). To reduce cross-reflections, optical circulators were connected in-advance of each network's connection to the WDM. Each of the circulators (Thorlabs, 6015-3-APC) provided greater than $-30 \mathrm{~dB}$ isolation from crossreflections such that no measurable reflections were detected during setup. Two circulators were used for each WDM input to reduce cross-reflections of light below levels measurable by the previously described photodiode detectors.

\section{Launch Collimator}

Once the beams were combined into a single fiber, the light was sent along a fiber optic cable to a 2-mm diameter (full-width half-maximum; FWHM) collimator (Thorlabs, RC02APC-P01). The collimated light was passed through a wedged window of 30 arcmin and was then sent into the open path area towards the collection optics. The wedged window was used to avoid optical resonance along the open path. Reflections of light between two parallel surfaces can cause optical interference fringes to appear in measured signals that could obscure the soot and $\mathrm{H}_{2} \mathrm{O}$ light attenuation signals.

\subsubsection{Reference Enclosure}

It has been shown that improving the thermal stability of the optical system reduces measurement drift (Schoonbaert et al., 2014). To reduce signal drift in this experiment all reference optics were housed inside a custom-made thermally-stabilized enclosure. The laser diodes themselves were 
left outside the enclosure since the diodes contained their own internal thermoelectric cooler units to ensure stability.

The enclosure had a thermoelectric cooler (TEC) unit mounted to it to monitor and control internal temperatures. The TEC unit (TE Technology, CP-121HT) was controlled by a temperature feedback controller (TE Technology, TC-36-25-RS232) connected to a thermistor located inside the enclosure to measure the internal air temperature. The custom-made steel enclosure had bulkhead cut-outs for optical and electrical connections to the internal components and was lined with insulating closed-cell foam. Figure 4.6 shows internal and external views of the enclosure. The TEC unit was placed on the lid of the enclosure. Placement of the TEC on top of the enclosure meant that cold air descended into the air-space of the enclosure as warm air from the electronics in the detectors rose, thus promoting air mixing for a less thermally-stratified enclosure. The TEC was set to hold a temperature of $18^{\circ} \mathrm{C}$ at a thermistor located at the base of the enclosure air-space. This target temperature was arbitrary since the necessary condition is that the temperature was stable throughout experiments. The selection of $18^{\circ} \mathrm{C}$ would ensure at least $4^{\circ} \mathrm{C}$ difference from typical lab temperatures. The temperature gradient would permit normal operation of the TEC. The thermistor in the enclosure was used as feedback to a digital PID control.
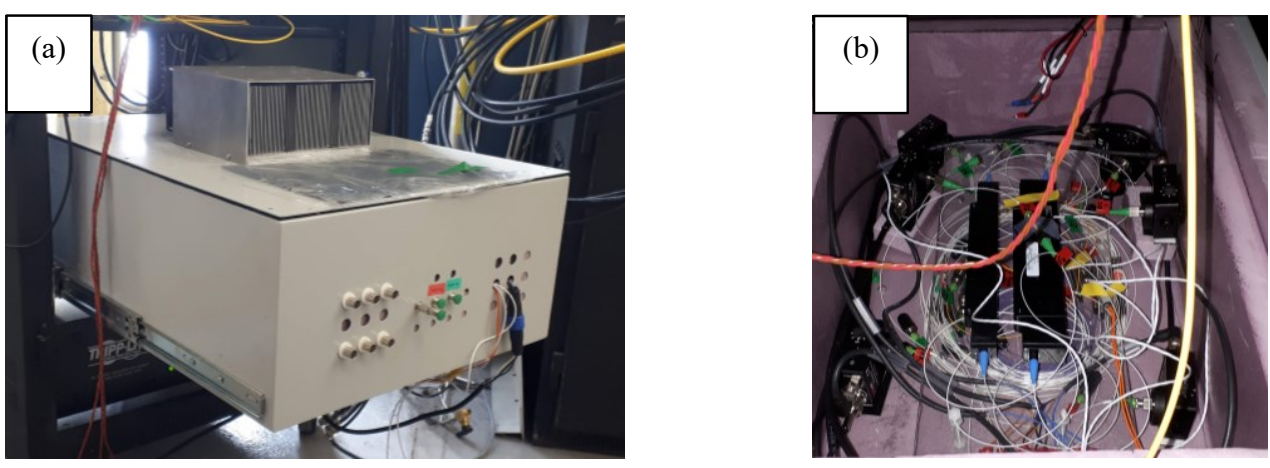

Figure 4.6: (a) Thermally-Stable Reference Enclosure and (b) Enclosure internal view. 


\subsubsection{Collection Optics}

After passing through the open path measurement area, the two-wavelength collinear beam of light was separated into its constituent wavelengths and focused onto separate photodiode detectors. The collection optics needed to focus the 2-mm diameter beam that had been subjected to beamsteering by the hot plume onto a 2-mm diameter detector area. The collection optics also needed to reject all other wavelengths of light that might be coupled into the system. Preliminary beamsteering experiments suggested the beam deflection by the hot plume would not exceed $4 \mathrm{mrad}$. Optics were sized on this basis and are shown schematically in Figure 4.7 and summarized in Table 4.2.

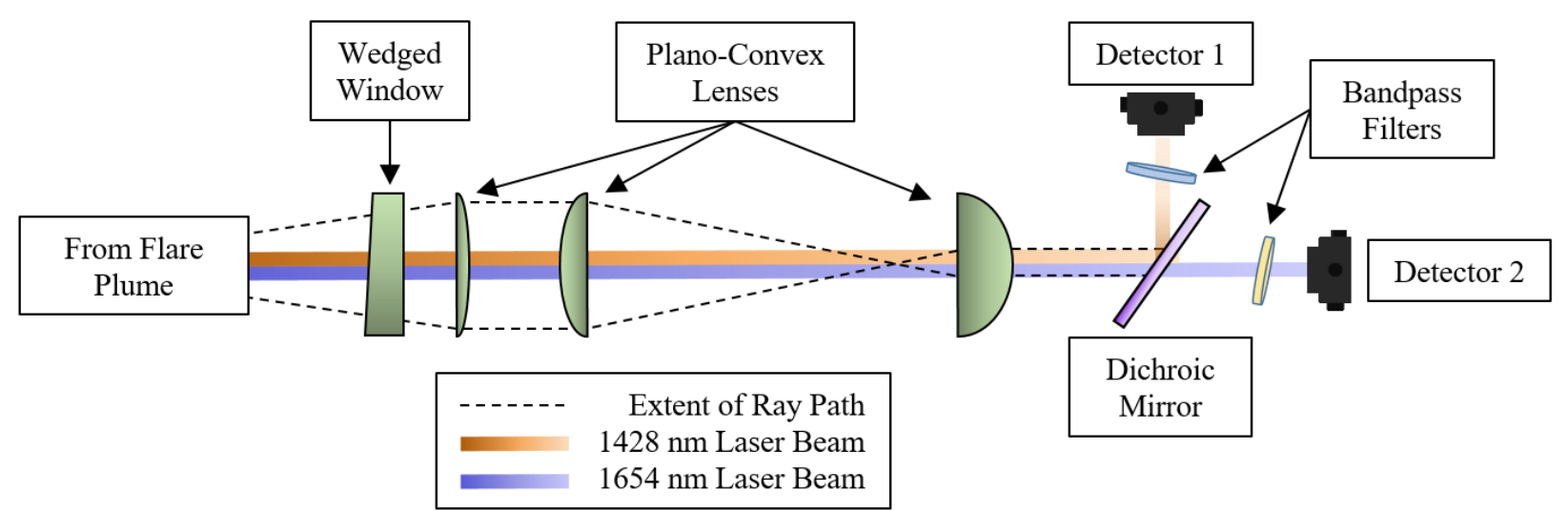

Figure 4.7: Schematic of collection optics. Dotted line indicates maximum extent of a steered beam.

The collection optics used plano-convex lenses to focus the beam by a 2:1 factor to ensure all steered light was captured by the detector element. A long-pass dichroic filter (DMLP1500R, Thorlabs) was used to split the two wavelengths. Wavelengths above the $1550 \mathrm{~nm}$ cut-off were transmitted through the filter while wavelengths below the cut-off underwent specular reflection. The separated beams were directed through bandpass filters centered around their respective center wavelengths to reject light from other sources and any light from the other laser that was not 
properly directed through the dichroic filter. All lenses were anti-reflective (AR) coated for maximum light transmission of the laser wavelengths.

Table 4.2: Major components of Collection Optics Assembly

\begin{tabular}{|c|c|c|c|}
\hline Component & Part No. & Provider & Notes/Description \\
\hline Bandpass Filter & FB1650-12 & Thorlabs & $\varnothing 1 "$ Bandpass Filter, CWL $=1650 \pm 2.4 \mathrm{~nm}, \mathrm{FWHM}=12 \pm 2.4 \mathrm{~nm}$ \\
\hline Plano-Convex & LA1805-C & Thorlabs & N-BK7 Plano-Convex Lens, $\varnothing 1 ", f=30 \mathrm{~mm}$, AR Coat: $1050-1700 \mathrm{~nm}$ \\
\hline Plano-Convex & LA1134-C & Thorlabs & N-BK7 Plano-Convex Lens, $\varnothing 1 ", f=60 \mathrm{~mm}$, AR Coat: $1050-1700 \mathrm{~nm}$ \\
\hline Plano-Convex & LA1978-C & Thorlabs & N-BK7 Plano-Convex Lens, $\emptyset 1 ", f=750 \mathrm{~mm}$, AR Coat: $1050-1700 \mathrm{~nm}$ \\
\hline Dichroic Filter & DMLP1500R & Thorlabs & $25 \mathrm{~mm} \times 36 \mathrm{~mm}$ Longpass Dichroic Mirror, 1500 nm Cut-On \\
\hline Wedged Window & WW11050-C & Thorlabs & $\varnothing 1 "$ Wedged N-BK7 Window, AR Coating: 1050 - 1700 nm \\
\hline Bandpass Filter & $87-868$ & Edmund & 1425nm Center, 25mm Diam. Hard Coated OD 4 50nm Bandpass Filter \\
\hline Detectors & PDA20CS & Thorlabs & InGaAs Amplified Detector, 800 - 1700 nm, 10 MHz BW, 3.14 mm² \\
\hline Detector Lens & C220TMD-C & Thorlabs & Mounted Aspheric Lens $\varnothing=7.22 \mathrm{~mm}, \mathrm{f}=11.00 \mathrm{~mm}, \mathrm{NA}=0.25 \mathrm{D}-\mathrm{ZK} 3$ \\
\hline Optomechanics & Assorted & Thorlabs & Assorted lens tubes, posts, optics mounts etc. \\
\hline
\end{tabular}

Although the collection optics were located sufficiently far above the flare to avoid coupling-in of light from the flame, the effect of hot plume gases along the line-of-sight was considered as a possible source of unwanted radiation into the system. Assuming plume temperatures of $1200 \mathrm{~K}$, Wien's displacement law would suggest peak emissivity of the plume would be in excess of $2400 \mathrm{~nm}$ (Howell et al., 2016) and are well beyond wavelengths detectable by the PDA detectors. This was confirmed in practice by measuring detector signals with lasers turned off during flare tests. Under these conditions no detectable intensity change was observed on the detectors.

\subsection{Acquisition/Control Hardware}

This section describes the data acquisition and control hardware station used to control laser outputs and read and process all detector signals. The hardware cabinet is shown in Figure 4.8 and Table 4.3 lists the main components which are discussed greater detail in subsequent sections. 

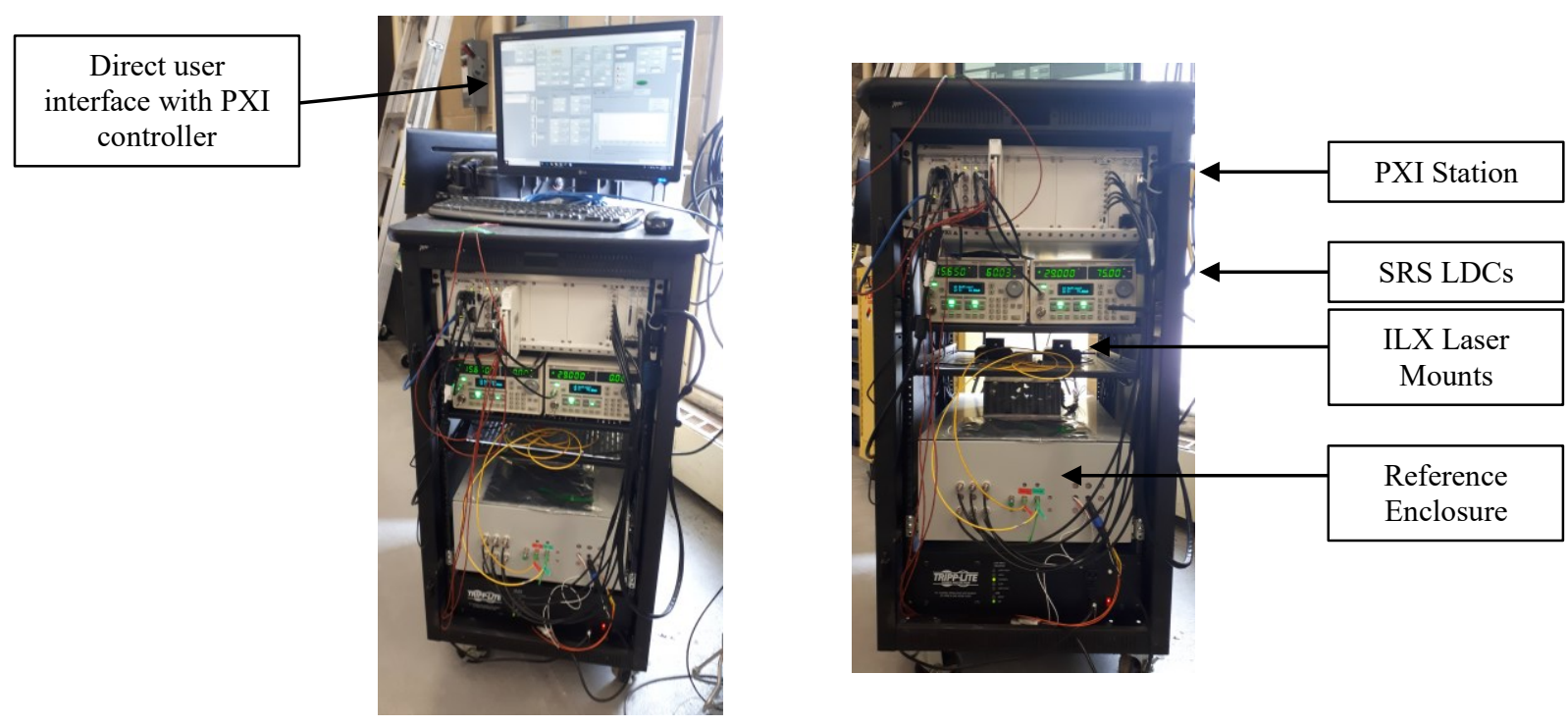

Figure 4.8: Remote base station which includes laser driving systems, reference optics in a thermally-stable enclosure, and the PXI control and acquisition system.

Table 4.3. Major components of the Acquisition \& Control Hardware Station

\begin{tabular}{|l|l|l|l|}
\hline Type & Part N & Provider & Notes/Description \\
\hline PXI Controller & PXIe-8840 & NI & 2.6 GHz Quad-Core Processor PXI Controller \\
\hline PXI Chassis & PXIe-1075 & NI & 18-Slot, Up to 4 GB/s PXI Chassis \\
\hline Frequency Generator & PXI-5406 & NI & $40 \mathrm{MHz}$ Bandwidth, 16-Bit PXI Waveform Generator \\
\hline Oscilloscope & PXIe-5172 & NI & $\begin{array}{l}100 \mathrm{MHz}, \text { 8-Channel, 14-Bit, Kintex-7 410T FPGA Reconfigurable PXI } \\
\text { Oscilloscope }\end{array}$ \\
\hline Temperature Module & $\begin{array}{l}\text { PXle-4357 } \\
\text { TB-4357 }\end{array}$ & NI & 20-Channel, 24-bit Temperature Input Module for RTDs \\
\hline Serial Interface & PXI-8432 & NI & RS-232 Digital Interface for Communication with TEC Unit \\
\hline
\end{tabular}

\section{PXI Controller and Chassis}

The fundamental components of the data acquisition and control station are the chassis (PXIe1075) and controller (PXIe-8840); both part of a National Instruments (NI) PXI PCI-based system.

The PXIe-8840 controller had an Intel Core i5 4400E processor (2.7 GHz dual core processor), $8 \mathrm{~Gb}$ RAM, $285 \mathrm{~Gb}$ hard disk and used a Windows 10 operating system. All control and acquisition commands were written in the LabVIEW environment directly on this controller. 
The 18-slot PXI chassis could accept and communicate with a wide variety of modular function cards made by NI. The backplane of the chassis had a $4 \mathrm{~Gb} / \mathrm{s}$ bandwidth and 10 and 100MHz reference clocks. The PXI chassis also featured dedicated trigger lines that allowed the modular cards to share start and reference clock triggers for tight time-synchronization.

\section{Frequency Generators}

The NI PXI-5406 Frequency Generator (FGEN) was responsible for generating the input voltage waveform to the SRS laser diode controller which in turn controlled the current-tuning of the laser. The FGEN card had a $40 \mathrm{MHz}$ bandwidth, 16-bit digital-to-analog waveform generator, which was capable of producing standard functions such as sine, square, triangle and ramp waveforms within a range of $+/-5 \mathrm{~V}$ via direct digital synthesis. The system used two FGENs; one for each laser diode controller. Although the FGEN was not used to scan the $1654 \mathrm{~nm}$ laser during experiments, it was used to precisely temperature-tune the diode away from methane absorption peaks prior to the experiment.

In practice, a triangle waveform from the FGEN was used to current-tune the $1428 \mathrm{~nm}$ laser across the absorption peaks of interest at a rate of up to $200 \mathrm{~Hz}$. Although the FGEN could produce higher sweep frequencies, the diode's current-tuned spectral window became more narrow and at $\sim 250 \mathrm{~Hz}$ the tuning window became too narrow to resolve both peaks.

\section{Data Acquisition - SCOPE/FPGA}

All detector signals were measured on an NI PXIe-5172 reconfigurable oscilloscope. The 8channel, 14-bit, Kintex-7 410 T Field-Programmable Gate Array (FPGA) oscilloscope allowed for simultaneous measurements at up to $250 \mathrm{MS} / \mathrm{s}$ per channel. The card had $-75 \mathrm{~dB}$ crosstalk isolation and customizable voltage range settings to maximize the usage of the 14-bit analog-to- 
digital converter. Unlike many FPGA modules offered by National Instruments, this module was only front-end customizable (voltage range, sampling rate, channels to read, etc.); all FPGA logic was internally generated. The oscilloscope was programmed to read in both open path detector signals and all reference signals at a rate of $1 \mathrm{MHz}$ per channel. This rate was limited by the acquisition capabilities of the PXI controller.

\section{Data Acquisition - Temperature}

The PXIe-4357 temperature input module was used in conjunction with the TB-4357 front-mount terminal block to allow for read-in of 3-wire RTDs that were used to measure temperatures in the thermally-stable reference enclosure as discussed in Section 4.4.2. This unit was used predominantly during the development process for the reference enclosure.

\section{Serial Interface}

The PXI-8432 serial interface module was used to communicate with the TEC temperature controller unit during initialization. Once the temperature controller feedback control characteristics and trip-off parameters were set, the unit was no longer used.

\subsubsection{Inference of Absorption}

Absorption/attenuation of light cannot be directly measured in a test medium. Instead, the light transmitted through the medium is compared to an estimate of the absorption-free light intensity to determine transmissivity as first described in Eq. (2.1). The absorption can then be inferred through Eq. (2.7). In the system presented in this thesis, a triangle voltage waveform was produced by the FGEN and sent to the LDC, which converts the voltage into a current waveform that powers the diode (i.e., the laser injection current). The changing injection current not only changes the diode temperature (and hence output wavenumber) but also the output power since an increased 
current causes more electron-hole recombinations and thus more recombinational radiation. Figure 4.9a shows example signals from the FRR, the reference cell, and the laser reference over one period of the current-tuning waveform; Figure $4.9 \mathrm{~b}$ shows the reference cell signal overtop an absorption-free intensity estimate. In the open path measurement, the absorption-free intensity is measured by placing a tube across the optical path and purging with $\mathrm{N}_{2}$ gas for several minutes to remove the light absorbing species (predominantly $\mathrm{H}_{2} \mathrm{O}$ ). In the reference cell, absorption-free intensity is estimated by scaling the laser reference signal to approximate expected absorption peak heights.
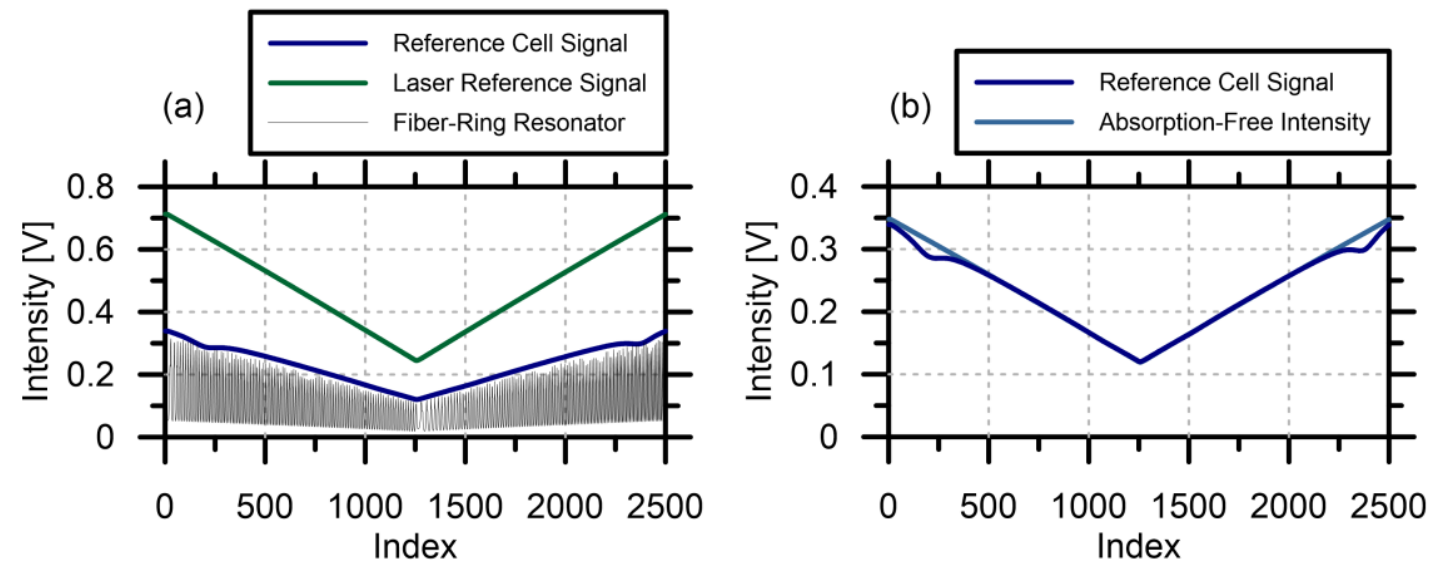

Figure 4.9: (a) Example reference cell, FRR, and laser reference transmitted intensity measurements over one period of the triangle current-tuning waveform, and (b) absorption-free intensity estimate compared with reference cell signal.

Normalizing the transmitted intensities by the absorption-free intensity returns the transmissivity which can yield absorption through Eq. (2.7). Figure 4.10 shows the transmissivity and absorption calculated for the signals of Figure 4.9a. The current-tuning sweep is mirrored about the sample index 1250 where the injection current passes the cusp of the triangle waveform and the current begins to increase. The mirrored absorption peak scans, referred to as lead (left) and lag (right) scans, are treated individually until wavenumbers are assigned to each. Treating lead and lag separate until the assignment of wavenumbers is done to ensure that any asymmetries that exist in 
the lead and lag scans (likely from diode hysteresis during heating/cooling) does not affect measured absorption feature when combining signals potentially out of phase. The lead and lag scans, once spectrally-resolved, are shown in Figure 4.11. These were then averaged to produce a single spectrum for analysis using the Nelder-Mead optimization scheme.

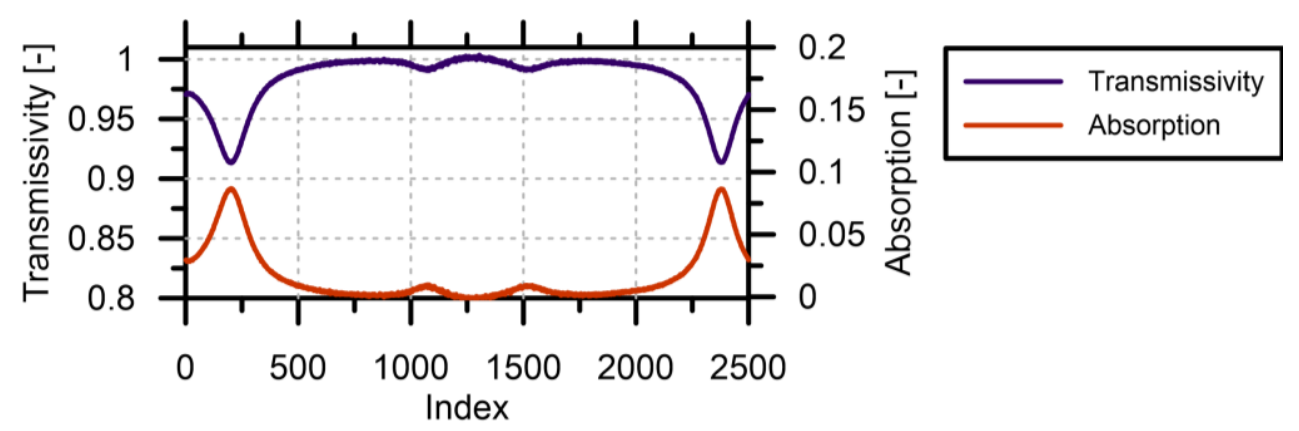

Figure 4.10: Mirrored scan of two absorption peaks during one current-tuning period of the laser shown in terms of both absorption and transmission.
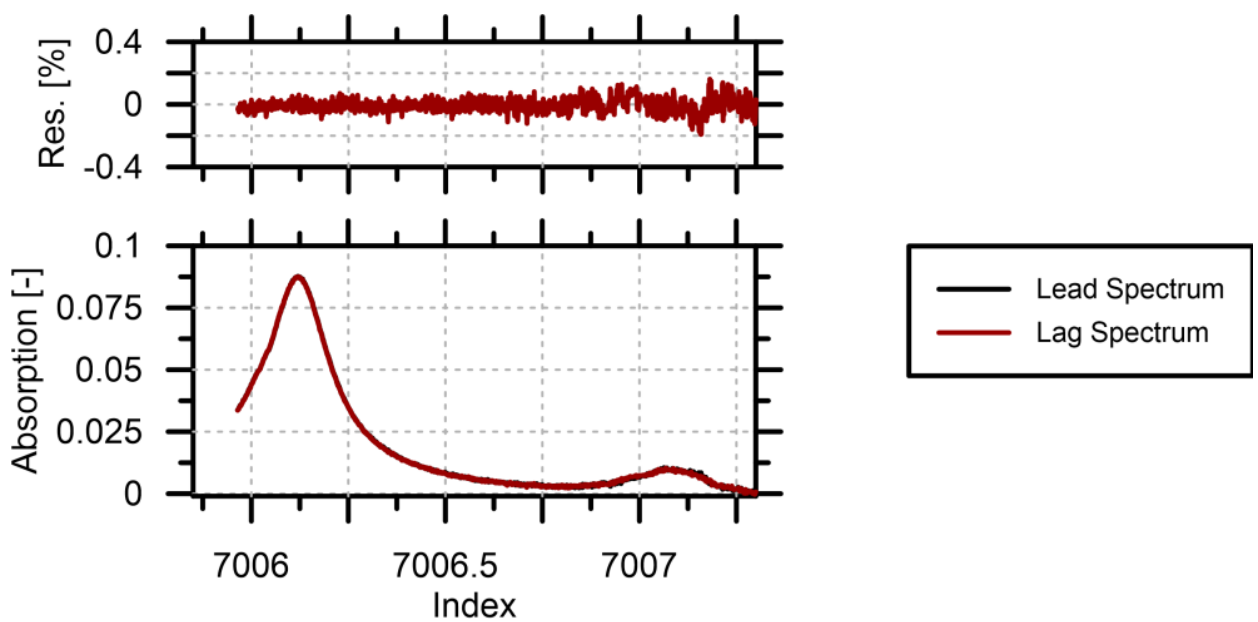

Figure 4.11: Lead-lag spectra compared on wavenumber basis for scan of Figure 4.10. The residual plot here shows the difference in absorption between lead and lag [\%].

For the $1654 \mathrm{~nm}$ laser, which is not current-tuned, the absorption-free intensity estimate is singlevalued but is otherwise measured similarly to the $1428 \mathrm{~nm}$ laser. Absorption-free intensity estimates were made prior to each experiment. 


\section{6 $\mathrm{H}_{2} \mathrm{O}$ Vapour Measurement Validation}

To validate the performance of the $\mathrm{H}_{2} \mathrm{O}$ spectral absorption system, measurements of $\mathrm{H}_{2} \mathrm{O}$ vapour were made in a flow-through cell filled with fluctuating concentrations of heated $\mathrm{H}_{2} \mathrm{O}$ vapour. This step was intended to be a calibration with known concentrations and temperatures being measured by the optical system, however the gas mixture supply unit was not sufficiently stable and large concentration fluctuations were observed. This is thought to be due to the mixer dispensing water in discrete droplets or condensation forming somewhere in the gas delivery lines. The experiment instead served the purpose of illustrating the closeness of fit of $\mathrm{H}_{2} \mathrm{O}$ vapour spectra in a controlled environment. Typical sum-of-squared-error (SSE) values resulting from the spectral fit in this experiment became the basis for setting limits on acceptable SSE values in the lab-scale flare experiments.

A controlled evaporator and mixer (CEM, Bronkhorst W-202A) was used to supply mixtures of distilled, evaporated $\mathrm{H}_{2} \mathrm{O}$ vapour with a carrier gas of $\mathrm{N}_{2}$ controlled by Coriolis and thermal mass flow controllers, respectively. The CEM supplied mixtures with fluctuating $\mathrm{H}_{2} \mathrm{O}$ volume fractions at a constant temperature through heated lines and into an insulated flow-through optical cell (Wavelength References, 20-cm path length). The optical cell was wrapped in heating blankets with J-type thermocouple feedback controls. Each test was conducted once the outlet temperature from the flow-through cell had stabilized. Figure 4.12 shows a schematic of the test cell. 


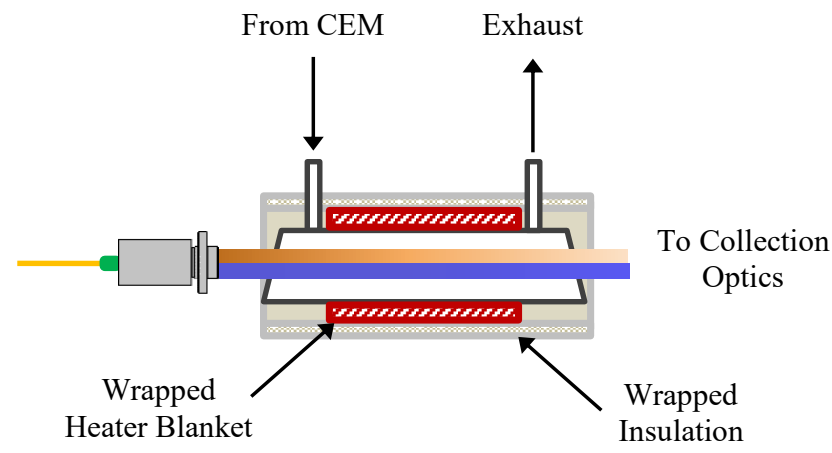

Figure 4.12: Schematic of flow-through reference cell with heater and wrapped insulation to maintain temperature of heated vapour sample.

By initially purging the cell with $\mathrm{N}_{2}$ gas, the absorption-free intensity of the open path lasers could be recorded; this became the baseline from which absorption was measured during subsequent tests. In this experiment, the column distribution was replaced with an assumed uniform distribution of temperature and concentration. The Nelder-Mead optimization algorithm then found the set of $\left\{T_{\text {cell }}, q_{\text {cell }}\right\}$ that most closely fit the measured $\mathrm{H}_{2} \mathrm{O}$ spectrum. Figure 4.13 shows some representative instantaneous results at three different temperature setpoints.

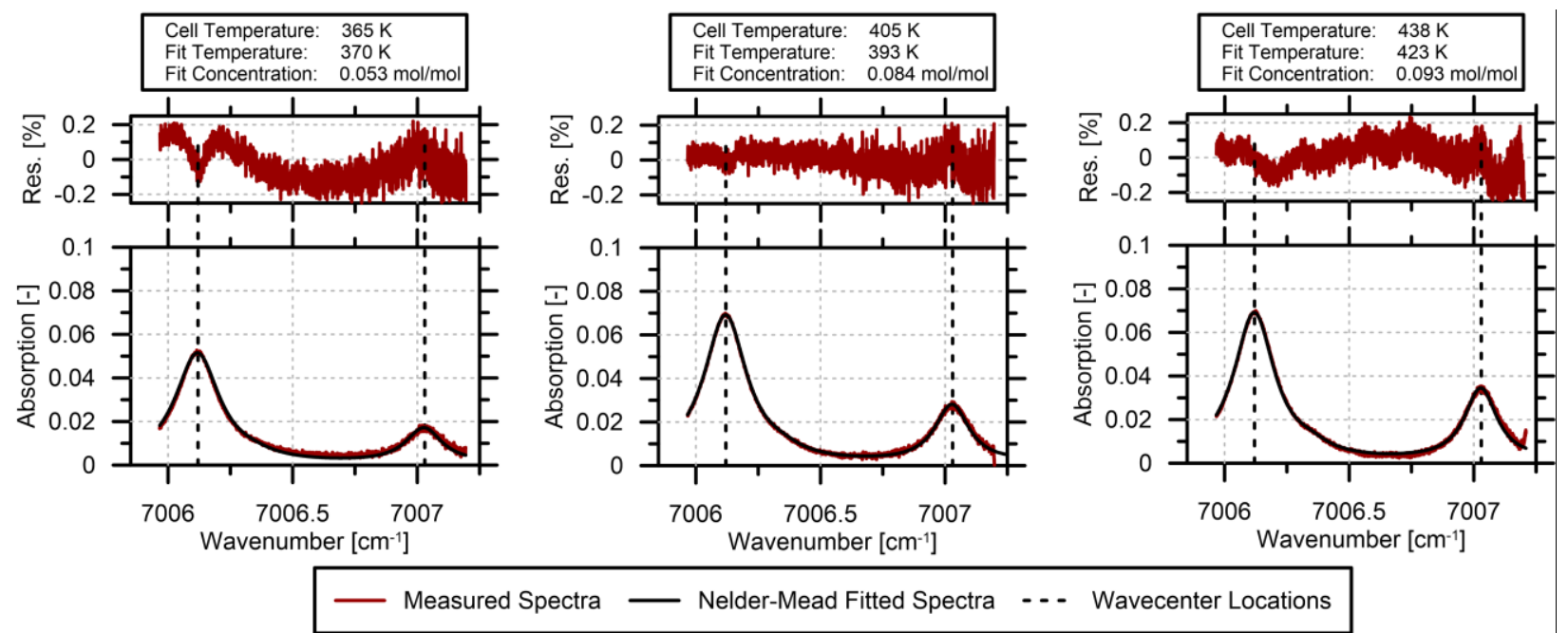

Figure 4.13: Samples of the spectral fit at three different temperature setpoints. Estimated temperatures closely agree with RTD-measured temperatures. 
Time-resolved $\mathrm{H}_{2} \mathrm{O}$ volume fraction results from the optical system are shown in Figure 4.14 for a 100-s sample during a test at $\sim 415 \mathrm{~K}$. Although the $\mathrm{H}_{2} \mathrm{O}$ vapour volume fraction varied from 0 to nearly $25 \%$, the temperature result was consistently within $\sim 20 \mathrm{~K}$ of the cell temperature. The only outliers in the temperature result corresponded to concentrations that were near zero. For these results, the spectral absorption approached zero and caused the spectral fit to return unrealistic values. This issue was not present during the flare tests since the ambient $\mathrm{H}_{2} \mathrm{O}$ vapour concentration was easily measured meaning spectral absorption would never reach zero.

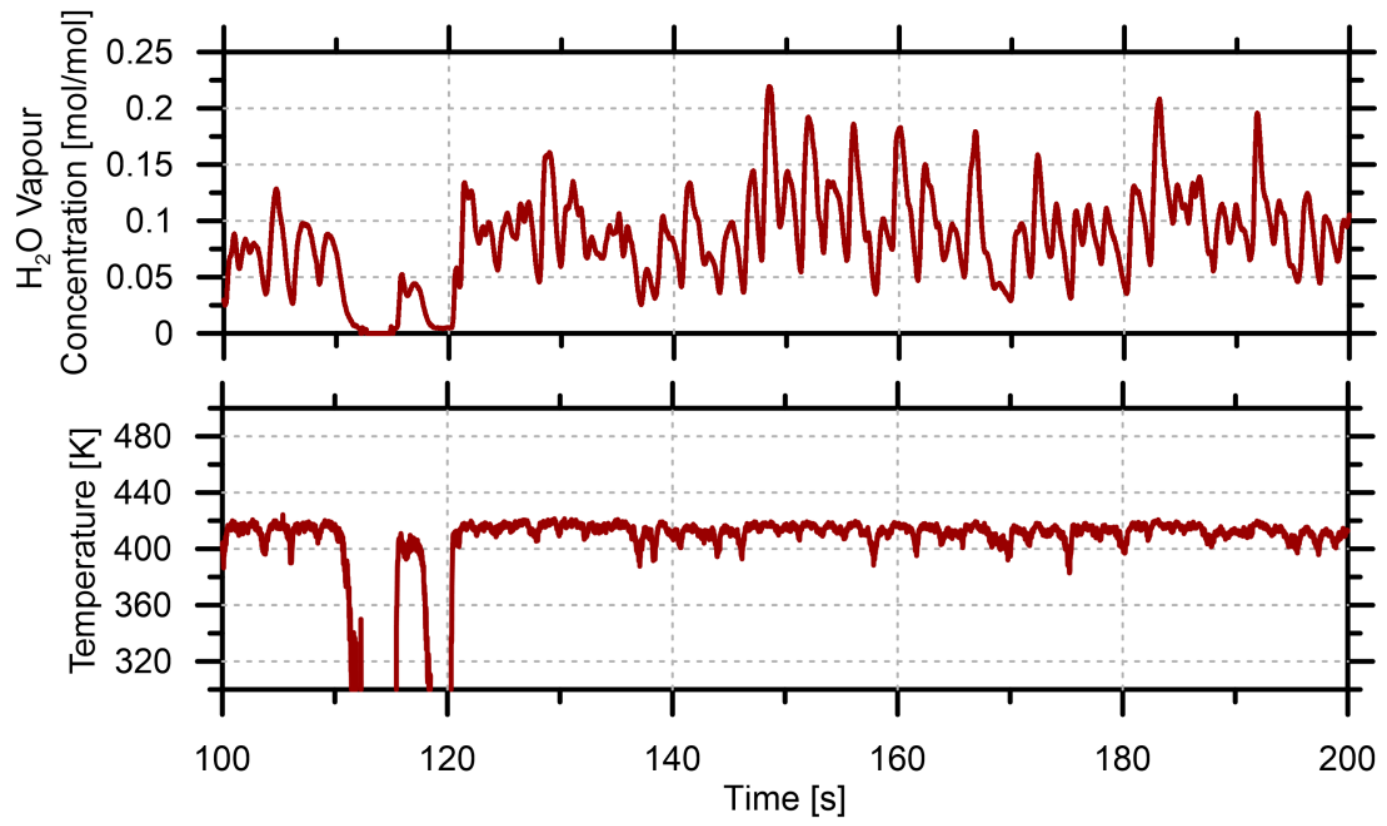

Figure 4.14: Time-varying results of $\mathrm{H}_{2} \mathrm{O}$ volume fraction and gas temperature from spectral measurements during flow-through tests at a set temperature of $\sim 415 \mathrm{~K}$.

In this controlled environment, the optical system appeared to be able to time-resolve the fluctuations in $\mathrm{H}_{2} \mathrm{O}$ volume fraction and return temperatures in the vicinity of the set temperatures. This experiment also demonstrated the closeness of the fit for the spectral measurements. Results from the Nelder-Mead column fit algorithm returned SSE values of less than $1.2 \times 10^{-4}$. This value is much higher than SSE values produced in the simulations (generally under $10^{-8}$ ). The difference in SSE values is likely due to non-uniformities of the gas in the flow-through cell and in attempting 
to fit real absorption spectra to theory. Although SSE values from this experiment cannot be used to directly assess error on the resulting $\mathrm{H}_{2} \mathrm{O}$ estimates, they were used as a guide to indicate best possible SSE values anticipated in the flare plume measurements.

\subsection{Flare Experiment}

Flare experiments were conducted at the Carleton University Flare Facility (CUFF) where custom gas mixtures characteristic of the upstream oil and gas industry are flared. The facility is shown in schematic in Figure 4.15. Custom combinations of $\mathrm{C} 1-\mathrm{C} 4$ alkanes, $\mathrm{C} 2$ and $\mathrm{C} 3$ alkenes, $\mathrm{CO}_{2}$ and $\mathrm{N}_{2}$ from pressurized gas cylinders and evaporated C5-C7 alkanes from the CEM (previously discussed in Section 4.6) can be combined and sent to a vertical flare stack to be combusted. The gas and liquid compounds are metered out by thermal mass flow controllers and Coriolis mass flow controllers, respectively. The evaporated liquid components are combined with a methane carrier gas and mixed with the remainder of fuel constituents.

The fuel mixtures are sent to a vertical flare nozzle (diameters of 25.4-mm, 50.8-mm and 76.2-mm internal diameter) and combusted in turbulent, buoyancy-driven, non-premixed flames. In the flare area, a three-axis traverse system is outfitted with a square-channel Invar hoop to mount the launch and collection sides of the optical measurement system. Fiber-optic cables and coaxial detector signal cables were run from the hoop to the remote control and acquisition station described in Section 4.5. The traverse was positioned such that the open path laser line was $2 \mathrm{~m}$ above the burner exit. The optical path between launch and collection optics (from the final window surface of the launch side to the first window of the collection optics), was $155 \mathrm{~cm}$. 


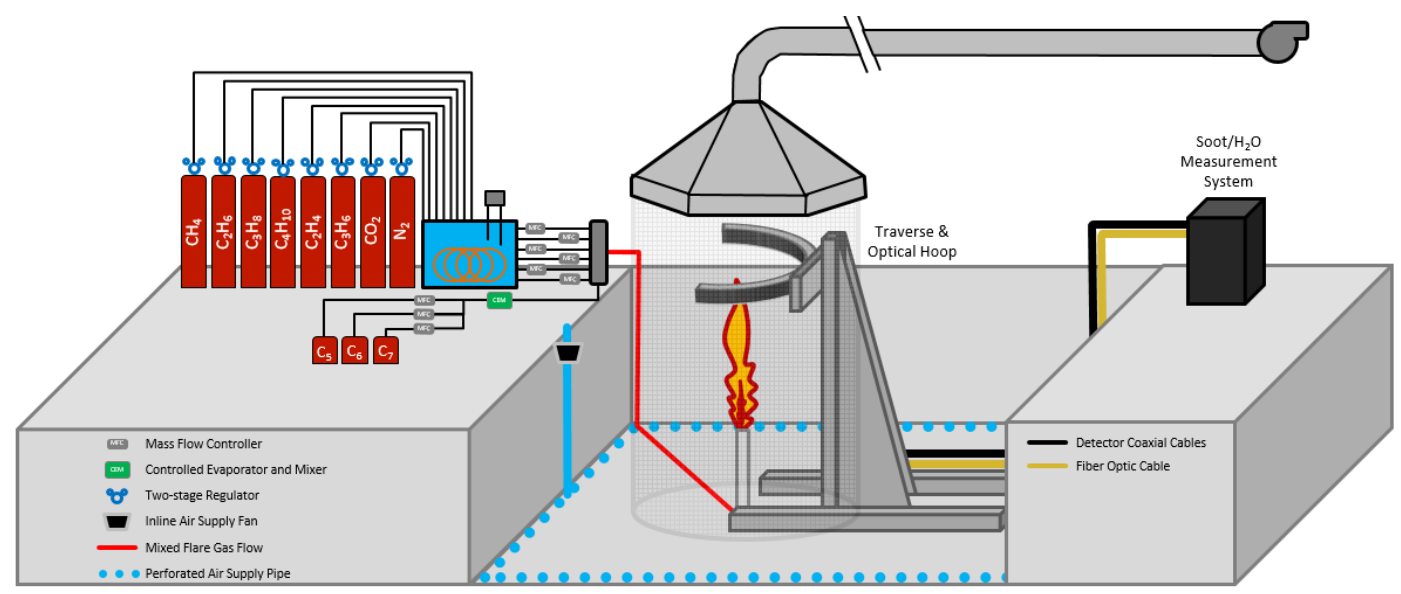

Figure 4.15: Schematic of Carleton University Flare Facility (CUFF).

Correlation experiments were completed for an array of fuel mixtures as outlined in Table 4.4.

Gas compositions were selected to follow Conrad \& Johnson (2019). These real-world and experimental mixtures ranged in $\mathrm{HHV}_{\mathrm{v}}$, which as previously discussed appears to be related to the sooting propensity of these types of fuels (Conrad \& Johnson, 2017; McEwen \& Johnson, 2012). A pure hydrogen case was also tested to assess the effects of beam steering on the interpreted results.

Table 4.4. Flare gas compositions in volume fraction [\%] studied in these experiments with their molecular weight (MW), volumetric higher heating value $\left(\mathrm{HHV}_{\mathrm{V}}\right)$ and molar carbon-hydrogen ratio (CHR).

\begin{tabular}{|l|l|l|l|l|l|l|l|l|l|l|l|l|l|}
\hline Fuel Name & $\mathbf{C 1}$ & $\mathbf{C 2}$ & $\mathbf{C 3}$ & $\mathbf{n C 4}$ & $\mathbf{i C 5}$ & $\mathbf{n C 6}$ & $\mathbf{n C 7}$ & $\mathbf{H}_{\mathbf{2}}$ & $\mathbf{N}_{\mathbf{2}}$ & $\mathbf{\mathbf { C O } _ { 2 }}$ & $\begin{array}{l}\mathbf{M W} \\
{[\mathbf{g} / \mathbf{m o l}]}\end{array}$ & $\begin{array}{l}\mathbf{H H V} \mathbf{v} \\
{\left[\mathbf{M J} / \mathbf{m}^{3}\right]}\end{array}$ & $\mathbf{C H R}$ \\
\hline $\begin{array}{l}\text { Bakken } \\
(\mathrm{BK})^{\mathrm{a}}\end{array}$ & 49.12 & 20.97 & 15.04 & 6.72 & 2.15 & 0.87 & 0.78 & 0 & 3.66 & 0.7 & 29.13 & 64.33 & 0.331 \\
\hline $\begin{array}{l}\text { Ecuador } \\
(\mathrm{EC})^{\mathrm{b}}\end{array}$ & 40.82 & 8.16 & 16.93 & 14.75 & 6.81 & 2.06 & 1.38 & 0 & 3.37 & 5.71 & 36.57 & 75.17 & 0.364 \\
\hline $\begin{array}{l}\text { Hydrogen } \\
\left(\mathrm{H}_{2}\right)\end{array}$ & 0 & 0 & 0 & 0 & 0 & 0 & 0 & 100 & 0 & 0 & 1.008 & 10.8 & 0 \\
\hline $\begin{array}{l}\text { Methane } \\
\left(\mathrm{CH}_{4}\right)\end{array}$ & 100 & 0 & 0 & 0 & 0 & 0 & 0 & 0 & 0 & 0 & 16.04 & 35.8 & 0.25 \\
\hline $\begin{array}{l}\text { Ethylene } \\
\left(\mathrm{C}_{2} \mathrm{H}_{4}\right)\end{array}$ & 0 & $100 \mathrm{c}$ & 0 & 0 & 0 & 0 & 0 & 0 & 0 & 0 & 28.05 & 62.96 & 0.5 \\
\hline
\end{tabular}

${ }^{a}$ Mean gas compositions from roughly 700 wells in Bakken region (Brandt et al., 2016)

b Selected gas composition from Ecuador's Orellana province (Conrad \& Johnson, 2017)

${ }^{c} \mathrm{C} 2$ contribution from ethylene $\left(\mathrm{C}_{2} \mathrm{H}_{4}\right)$ 
Table 4.5 shows the experimental matrix for the correlation measurements which includes a range of fuels, burner diameters, and fuel flow rates. The matrix was intended to cover a range of $\mathrm{HHV}_{\mathrm{v}}$ and carbon-hydrogen ratios (CHR) to assess the potential limits of the optical system. Additionally, a 50-SLPM ethylene flare was produced on the 76.2-mm burner to make measurements of soot attenuation to infer the soot extinction coefficient ratio which was necessary to isolate $\mathrm{H}_{2} \mathrm{O}$ spectral absorption from broadband soot attenuation as discussed in Chapter 2. Each flare was run for up to 20 minutes in advance of optical measurements to ensure the burner nozzle had reached a stable temperature.

Table 4.5: Flow Conditions for Correlation Measurements

\begin{tabular}{|c|c|c|}
\hline Fuel Name & $\begin{array}{c}\text { Burner Diameter } \\
{[\mathrm{mm}]}\end{array}$ & $\begin{array}{c}\text { Flow Rates } \\
{[\text { [SLPM] }}\end{array}$ \\
\hline Hydrogen & 25.4 & 150 \\
\hline Methane & 25.4 & 50,100 \\
\hline \multirow{2}{*}{ Bakken } & 25.4 & 40,60 \\
\cline { 2 - 3 } & 76.2 & 40,60 \\
\hline \multirow{2}{*}{ Ecuador } & 25.4 & 40,60 \\
\cline { 2 - 3 } & 76.2 & 40,60 \\
\hline Ethylene & 76.2 & 30,50 \\
\hline
\end{tabular}

a Standard liters per minute referenced at $0^{\circ} \mathrm{C}, 101,325 \mathrm{~Pa}$

\subsection{Experimental Procedures and Data Flow}

In advance of each experiment, absorption-free laser intensity estimates were made to account for any changes in laser output power changes or changes in alignment of the optics from thermal expansion during previous tests. These estimates were the basis from which all absorption signals were referenced. A tube with open ends was mounted between the launch and collection optics and purged with $\mathrm{N}_{2}$ gas. This removed all $\mathrm{H}_{2} \mathrm{O}$ vapour absorption from the optical path which was the dominant spectral absorption at the operating wavelengths. The purge was run for several minutes and the transmitted laser intensity averaged over $5 \mathrm{~s}$. 
Once the absorption-free intensity was measured, it was possible to measure the ambient $\mathrm{H}_{2} \mathrm{O}$ volume fraction in the lab by inspecting the absorption spectra. This was recorded several times throughout the experimental program and was used as an input to the column distribution Nelder-Mead algorithm as described in Section 2.1.2. Estimates of ambient $\mathrm{H}_{2} \mathrm{O}$ volume fractions were made periodically throughout the testing program, which ran from Feb $9^{\text {th }}$ to $11^{\text {th }}, 2019$. Figure 4.16 shows the measured ambient $\mathrm{H}_{2} \mathrm{O}$ results over the measurement campaign. The $25 \%$ insensitivity band illustrates the permissible variation in the ambient $\mathrm{H}_{2} \mathrm{O}$ value without affecting the path-averaged $\mathrm{H}_{2} \mathrm{O}$ results based on the simulations discussed in Section 3.3.

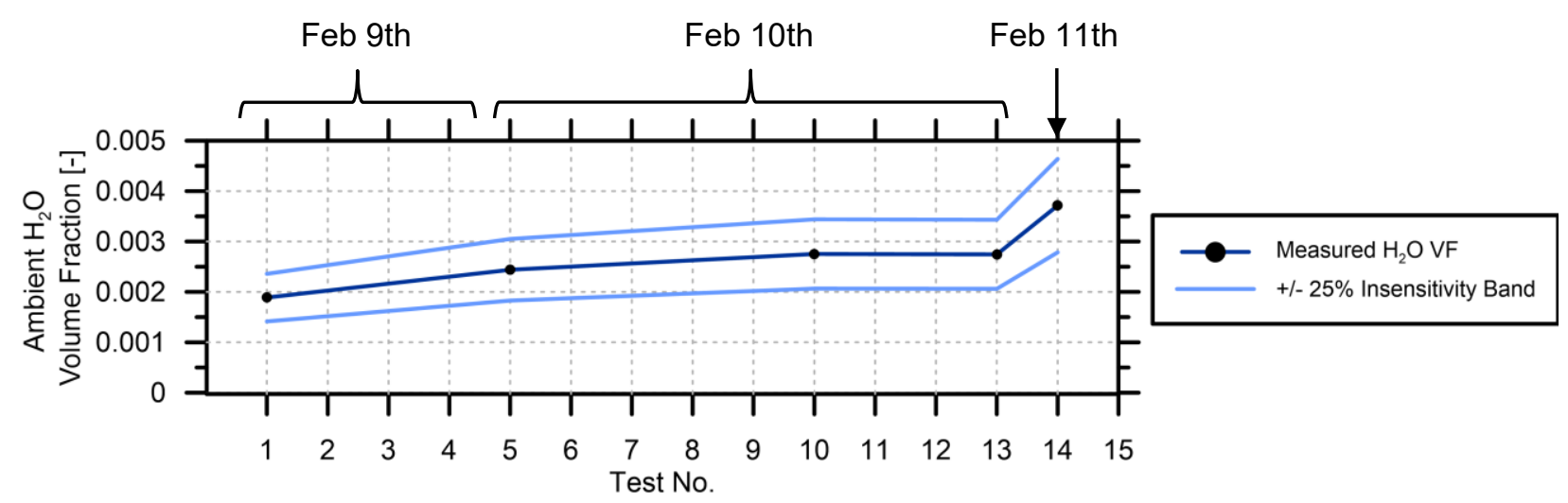

Figure 4.16: Measured ambient $\mathrm{H}_{2} \mathrm{O}$ volume fraction measured between flare experiments

Some in-line data processing was required on the 8-channel, $1 \mathrm{MHz}$ sampling system to permit continuous measurements. Real-time processing steps included removal of measured detector dark current voltages, averaging of lead- and lag-sweeps of the triangle waveform of the laser, assignment of wavenumber to each sample index (using the reference cell peaks and FRR signals as outlined in Section 4.4.1), and normalizing of open path detector signals by the absorption-free intensity to obtain absorption results. At the end of each flare experiment, the fuel flow was stopped and the fuel supply line was purged with $\mathrm{N}_{2}$ gas. Once this was complete, the absorption signals of both lasers were measured to determine whether thermal expansion of either the hoop 
or the optics themselves had affected beam alignment. A change in beam alignment would likely result in a loss of light and be registered as increased presence of soot. If residual attenuation after the flare test was observed, implying there was change in alignment, the test data were rejected and the test rerun.

Data written to hard disk consisted of open path absorption signals and wavenumber assignments to each value for each laser sweep. The datasets from each experiment were postprocessed in a Maple computing environment where SVF estimates were made and isolated $\mathrm{H}_{2} \mathrm{O}$ spectra were fit to column distribution spectra.

\subsection{Beam Steering Effects}

Issues related to optical beam steering were anticipated during measurements where high thermal gradients and correspondingly high density gradients would likely be present through the plume. Preliminary tests with visible lasers indicated bulk beam steering magnitudes of roughly $4 \mathrm{mrad}$, however the effects of beam steering needed to be quantitatively investigated.

Beam steering had the potential to severely limit the measurement abilities of this optical system. The system was meticulously aligned prior to tests in the flare facility, however, if the beam of light passing through the hot flare plumes was refracted enough, it would be possible to lose light in the collection optics. Any loss or gain of light at the detectors would necessarily be interpreted as fluctuations in soot presence along the optical path with no method for distinguishing the two. Although the collection optics were designed with this consideration, some light loss due to beam steering would still persist since each optical component likely had some incident-angle dependence on its transmissivity. This issue could also be amplified in cases where beam steering provokes some light loss that is not equal along each laser line. In other words, if an incident, 
steered beam entered the collection optics (assuming the two beams were still collinear upon entry into the collection optics), after being split at the dichroic filter each beam may be subjected to different degrees of light loss before being focused onto their respective detectors.

This section attempts to quantify the beam steering effects on the system by measuring both the single-line light loss and the differences in light loss between the two wavelengths. The latter would be predominantly caused by non-identical alignments of the incident beam and the two detectors. By burning pure hydrogen in the lab, light attenuation caused by beam steering could be measured in the absence of soot since hydrogen flares would only produce $\mathrm{H}_{2} \mathrm{O}$ vapour. Measurements were made on a hydrogen flare at 150 SLPM on the 25.4-mm diameter burner.

By observing the signal attenuation of the $1654 \mathrm{~nm}$ laser over a period of 50 seconds of the hydrogen flare test, a histogram of signal attenuation was constructed as shown Figure 4.17. Figure 4.17 also indicates the path-averaged SVF that would be inferred from such a magnitude of attenuation.

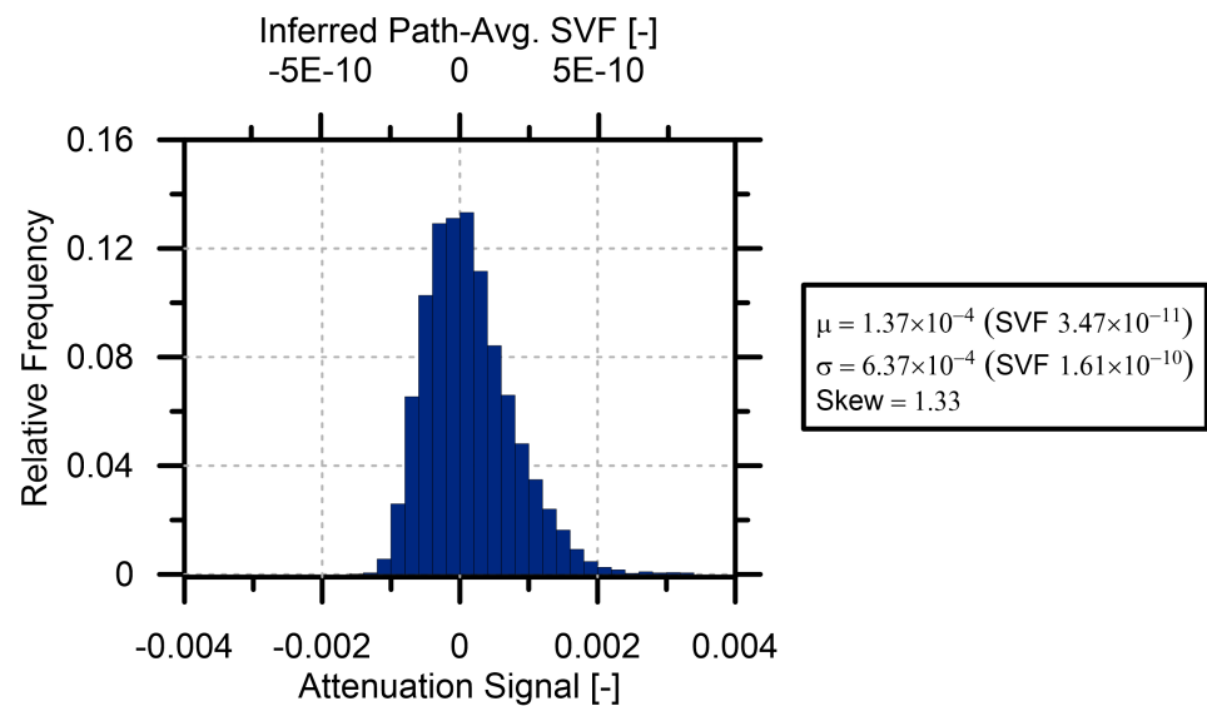

Figure 4.17: Light attenuation due to beam steering and inferred SVF presence caused by the attenuation. 
The signal attenuation seen in Figure 4.17 can be compared to corrupting noise that was assumed in the simulations of Chapter 3. By solely measuring laser and detector noise, a normally distributed noise signal was generated with no mean offset and a standard deviation of $5 \times 10^{-5}[-]$. Here, the signal attenuation due to single-line beam steering coupled with laser and detector noise had a standard deviation of $6.37 \times 10^{-4}$, more than an order of magnitude larger. This shows that beam steering has a much more significant effect on measurement uncertainty. Additionally, there appears to be a bias of the measurement towards light loss (positive signal attenuation, resulting in a positive mean value) meaning results of SVF will tend to bias high. For reference, the system was designed to detect soot attenuation down to at least $0.5 \%$. At this minimum design condition, the signal-noise ratio (SNR) would be $\sim 4$ for $95 \%$ of the measurements. For the measurement results discussed in Chapter 5, nearly $90 \%$ of the individual results had soot attenuation exceeding $0.5 \%$ and thus had an SNR greater than 4 (median value of attenuation being $2.6 \times 10^{-9}$ representing an SNR of $\sim 8$ ). Although some biasing of SVF may still have been caused by beam steering, it should not have affected correlation results.

The asymmetry in light loss due to beam steering between the two lines is more difficult to measure because although the $1428 \mathrm{~nm}$ laser may be tuned to a spectral location of minimized $\mathrm{H}_{2} \mathrm{O}$ absorption, the $\mathrm{H}_{2} \mathrm{O}$ absorption will never truly reach zero and will be fluctuating. This will tend to make fluctuations in light loss due to beam steering for the two lasers more difficult to quantify. Figure 4.18 and Figure 4.19 show theoretical $\mathrm{H}_{2} \mathrm{O}$ absorption spectra under a range of typical $\mathrm{H}_{2} \mathrm{O}$ volume fractions and temperatures intended to bound the possible fluctuations of $\mathrm{H}_{2} \mathrm{O}$ absorption at the minimum. The selected conditions represent path-averaged values of $\mathrm{H}_{2} \mathrm{O}$ volume fraction and temperature that exceed values measured in Chapter 5. Although spectral modelling is less reliable far away from the absorption peaks, and hence the absolute absorption 
expected should be considered approximate, Figure 4.18 and Figure 4.19 suggest that fluctuations in $\mathrm{H}_{2} \mathrm{O}$ absorption should not exceed $0.03 \%$ during experiments.

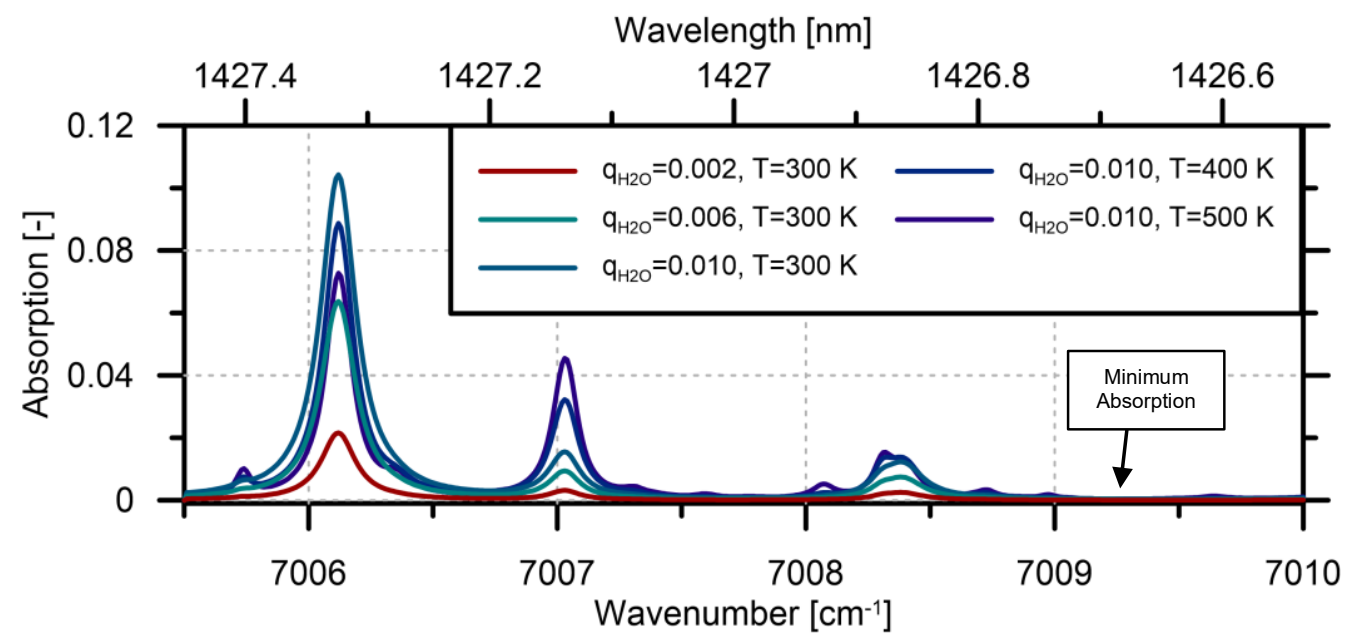

Figure 4.18: Theoretical $\mathrm{H}_{2} \mathrm{O}$ absorption at different gas conditions within the range of the $1428 \mathrm{~nm}$ laser. $\mathrm{H}_{2} \mathrm{O}$ absorption is at a minimum at $1426.686 \mathrm{~nm}\left(7009.25 \mathrm{~cm}^{-1}\right)$.

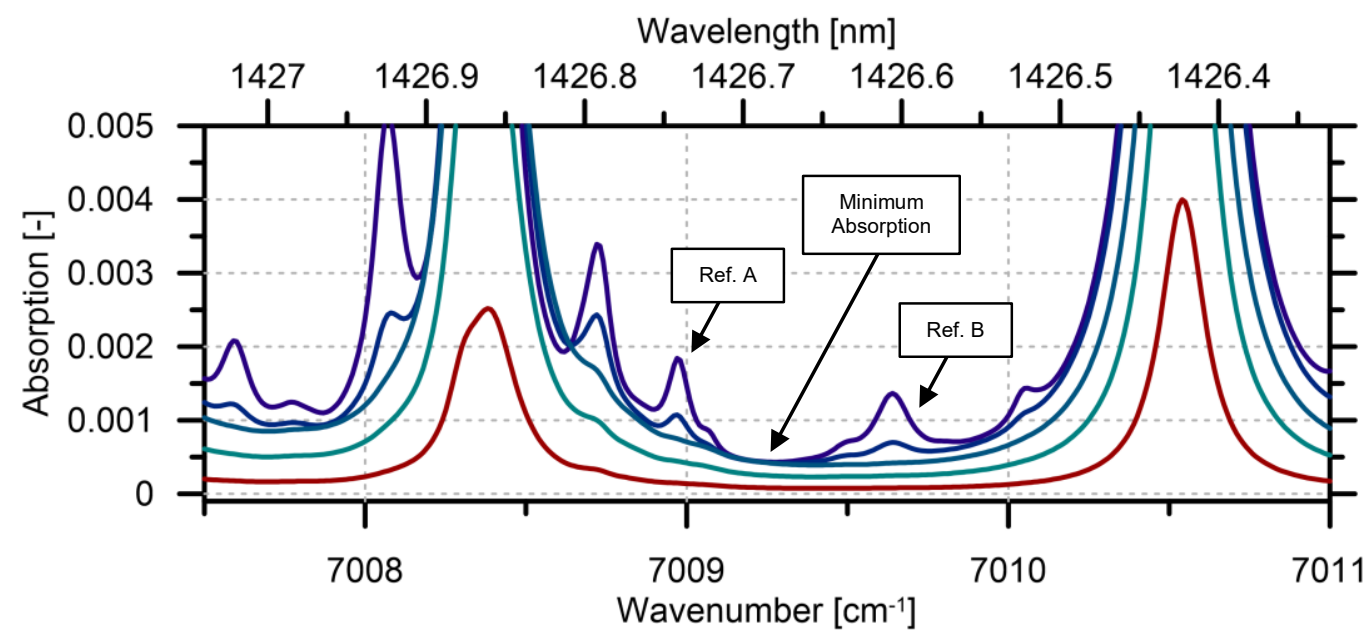

Figure 4.19: Zoomed-in view of Figure 4.18 indicating the spectral region of minimum $\mathrm{H}_{2} \mathrm{O}$ absorption. Ref. A and B were used during CEM validation experiments to precisely position the laser output.

Precise positioning of the laser at $1426.686 \mathrm{~nm}\left(7009.25 \mathrm{~cm}^{-1}\right)$ was not performed directly during flare experiments as there is no strong absorption peak at that location to reference, such that the minimum location could not be discerned at ambient temperature. Instead, absorption 
measurements from the tests performed in Section 4.6 were heavily-averaged at the high temperature setting. At the nominal $200^{\circ} \mathrm{C}$ cell temperature, the absorption peaks labelled Reference A and B in Figure 4.19 were measurable and by recording the necessary laser diode settings to position the laser between Ref. A and B peaks, the precise spectral location could be tuned to during the flare experiments.

Measurements of signal attenuation with the $\mathrm{H}_{2} \mathrm{O}$ detection laser positioned statically at $1426.686 \mathrm{~nm}$ allowed for an estimate of two-line beam steering effects to be investigated. Figure 4.20 shows a histogram of point-by-point signal attenuation difference between the two laser signals. Although this result shows that two-line beam steering influences are present in the system, generally the signals did not differ by more than $0.25 \%$ and are roughly centered about zero implying the two-line beam steering is not biased towards attenuating one wavelength more than the other. During flare experiments, soot attenuation typically reaches $3 \%$ for the Bakken and Ecuador mixtures meaning this effect is limited to an $8 \%$ relative influence on signals where the two laser signals are compared (i.e., $\mathrm{H}_{2} \mathrm{O}$ detection signals). Although this could be detrimental to the $\mathrm{H}_{2} \mathrm{O}$ spectral analysis, the ability to compare measured spectra with theoretical spectra will allow for identification of spectral measurements where beam steering has produced impossible results for $\mathrm{H}_{2} \mathrm{O}$ spectra. This was quantified by a cutoff SSE value which removed spectral fits with high absorption residuals indicating a sufficient fit could not be obtained. 


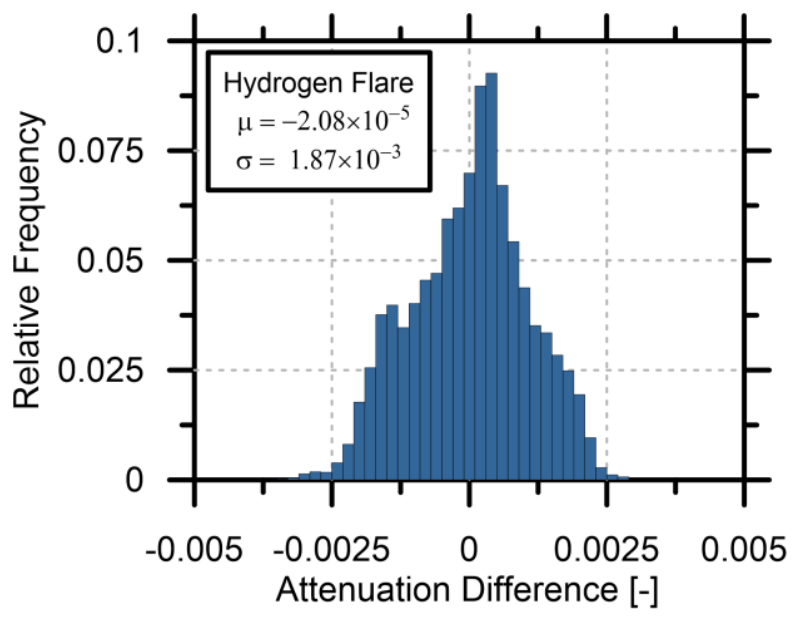

Figure 4.20: Difference in signal attenuation between laser signals which represents the asymmetry of beam alignment provoked by beam steering. 


\section{Chapter 5 Results \& Discussion}

\subsection{Overview}

This chapter presents the results of the experiments conducted at the Carleton University Flare Facility (CUFF) to measure correlations in time-resolved, path-averaged estimates of $\mathrm{H}_{2} \mathrm{O}$ and soot. Experiments were first conducted to estimate soot optical property ratios between the selected wavelengths as a necessary step to isolating $\mathrm{H}_{2} \mathrm{O}$ absorption peaks for simultaneous measurements. The soot property experiment was further analyzed to characterize the effective timescales of path-averaged measurements in the turbulent flare plumes. Experiments were then conducted to measure the correlation of path-averaged $\mathrm{H}_{2} \mathrm{O}$ and soot in the plume for different fuels. Correlation results are used to illustrate implications for short-duration flare emission sampling techniques.

\subsection{Characterization Experiments}

\subsubsection{Soot Extinction Coefficient Ratio}

As discussed in Chapter 2, the ratio of soot extinction coefficients between the measurement wavelengths of $1428 \mathrm{~nm}$ and $1654 \mathrm{~nm}$ was required to isolate narrow $\mathrm{H}_{2} \mathrm{O}$ absorption spectra from broadband light attenuation by soot. Since previous studies do not agree on soot optical properties at either wavelength, the properties were assumed at $1654 \mathrm{~nm}$ and an experiment was conducted to measure the ratio of extinction coefficients between $1428 \mathrm{~nm}$ and $1654 \mathrm{~nm}$.

To measure soot attenuation in isolation from $\mathrm{H}_{2} \mathrm{O}$ vapour absorption, the $1428 \mathrm{~nm}$ laser was temperature-tuned to $1426.686 \mathrm{~nm}\left(7009.25 \mathrm{~cm}^{-1}\right)$, the spectral region within the available laser range where $\mathrm{H}_{2} \mathrm{O}$ absorption was minimized. The $1654 \mathrm{~nm}$ laser was tuned away from methane absorption (as noted in Section 4.4.1) but was otherwise not significantly affected by gas 
phase species absorption. As discussed in Section 4.9, the anticipated fluctuations in $\mathrm{H}_{2} \mathrm{O}$ absorption at this spectral region would not exceed $0.03 \%$. To further ensure that soot attenuation was minimally obscured by $\mathrm{H}_{2} \mathrm{O}$ absorption fluctuations, a heavily-sooting ethylene flare was produced at 50 SLPM on the 76.2-mm diameter burner. The ethylene flare produced optical attenuation up to $\sim 20 \%$ which is nearly three orders of magnitude higher attenuation than that produced by $\mathrm{H}_{2} \mathrm{O}$ absorption. Under these conditions any $\mathrm{H}_{2} \mathrm{O}$ absorption fluctuations were negligible. The soot extinction coefficient ratio was estimated through the use of Eq. (2.16),

$$
\frac{\ln \left(\tau_{1}\right)}{\ln \left(\tau_{2}\right)}=\frac{K_{e_{1}}}{K_{e_{2}}} \frac{\lambda_{2}}{\lambda_{1}}
$$

where subscripts 1 and 2 represent wavelengths of 1428 and $1654 \mathrm{~nm}$, respectively, and the transmissivities $\tau$ were measured. This expression is a simple rephrasing of Eq. (2.9) where a ratio of attenuation at the two wavelengths is taken and is related to soot optical properties following Rayleigh-Debye-Gans theory for fractal aggregates. Soot attenuation measurements were taken at a rate of $500 \mathrm{kHz}$ on both laser signals.

Figure 5.1 shows a histogram of the distribution of instantaneous soot extinction coefficient ratio estimates during a 50-second period of ethylene flare measurements. The mean soot extinction coefficient ratio $\left(K_{e_{(1428 n m)}} / K_{e_{(1654 n m)}}\right)$ was 0.964 . This result is bounded by ratios calculated from available extinction experiments from the literature (originally discussed in Chapter 2 and shown in Figure 5.1) and most closely resembles the result calculated using data from Krishnan et al. (2001) whose extinction coefficient findings would suggest a ratio of 0.969 between these wavelengths. Since beam steering will cause some loss of light for each wavelength, the measured value of 0.964 may still include some bias. From Section 4.9 each signal attenuation 
is biased on the order of $\pm 0.12 \%$ due to light loss from beam steering. If the typical soot attenuation is on the order of at least $6 \%$ and if the two beams are biased due to beam steering, one positive, one negative, then this accounts for at most $4 \%$ of the overall attenuation and would bias the extinction coefficient ratio by \pm 0.013 .

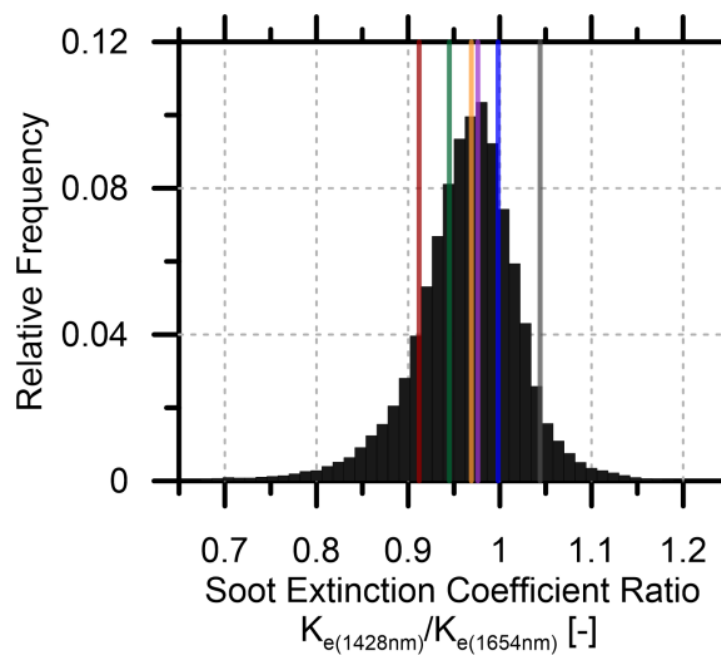

Figure 5.1: Distribution of estimated $K_{e}$ ratio between 1428 and $1654 \mathrm{~nm}$ during ethylene flare test. The measured mean soot extinction coefficient ratio was 0.964 with a standard deviation of 0.065 .

\subsubsection{Turbulent Plume Timescales}

At the design stage of this optical measurement system, necessary sampling rates in the turbulent plumes were not known and could only be estimated. To ensure that the sampling rates of the designed system were sufficient to time-resolve the path-averaged measurement, an autocorrelation study was conducted on the aforementioned soot attenuation measurements from the ethylene flare. This was performed to estimate the effective timescales of the path-averaged measurement.

The autocorrelation function was calculated using 1-minute durations of the soot attenuation measurement on the $1654 \mathrm{~nm}$ laser collected at $500 \mathrm{kHz}$. The results of seven such intervals are plotted in Figure 5.2 along with the mean result. The effective timescale is the longest 
time between two measurements where the history of one influences the next. O'Neill et al. (2004) suggest that the timescales can be approximated by the point at which the autocorrelation function first reaches zero. Taking the mean timescale estimates from each interval in Figure 5.2, the apparent timescale of the path-averaged measurements is $\sim 0.38 \mathrm{~s}$. This is an indication of the timescale governing large-scale motion and entrainment of air into the plume, akin to the integralscale of turbulence. However, there are smaller, shorter timescale fluctuations that are also relevant to the present experiment.

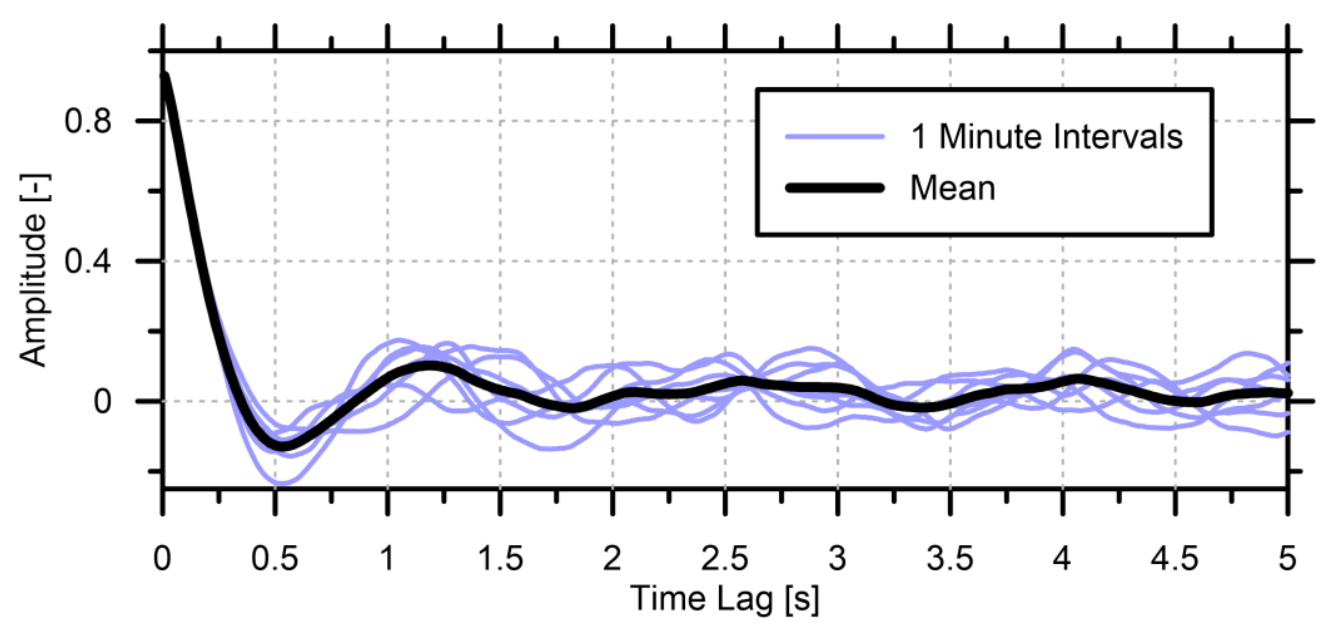

Figure 5.2: Autocorrelation function of soot attenuation signals for seven 1-minute datasets. The mean effective timescale for measurements in the plume appears to be $\sim 0.38 \mathrm{~s}$.

Figure 5.3 shows an excerpt of the raw soot attenuation data. Over the 10 -second period shown there are some broad attenuation fluctuations which drive the autocorrelation function. However, the higher resolution view shown in Figure 5.4 reveals the scale of the shortest fluctuations. These measurements, taken at a rate of $500 \mathrm{kHz}$, were able resolve fluctuations of sub-millisecond durations. Although the soot attenuation measurement can resolve these sub-millisecond fluctuations, the system is limited by the $200 \mathrm{~Hz}$ maximum sweep rate of the $1428 \mathrm{~nm}$ laser. As previously discussed, sweep rates in excess of $200 \mathrm{~Hz}$ narrowed the spectral scan width to the point where the width was not sufficient to observe both $\mathrm{H}_{2} \mathrm{O}$ absorption peaks. With a sweep period of 
$5 \mathrm{~ms}$, the $\mathrm{H}_{2} \mathrm{O}$ absorption peak measurements will not be able to resolve sub-millisecond fluctuations of $\mathrm{H}_{2} \mathrm{O}$. In Figure 5.4, although the soot attenuation is highly time-resolved, this same period will only yield 3 spectral measurements for $\mathrm{H}_{2} \mathrm{O}$. Future work on improving the timeresolution of the optical system is discussed in Section 6.1.

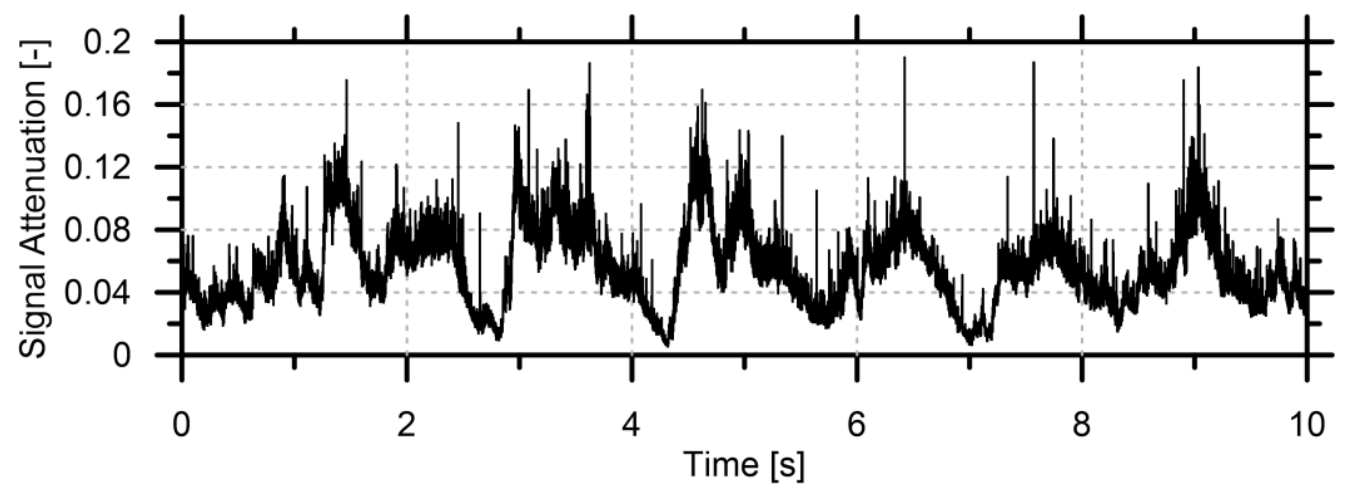

Figure 5.3: Time-resolved soot attenuation signal over 10 seconds. Bulk fluctuations of soot attenuation most heavily weight the autocorrelation function and result in apparent timescales of $0.38 \mathrm{~s}$.

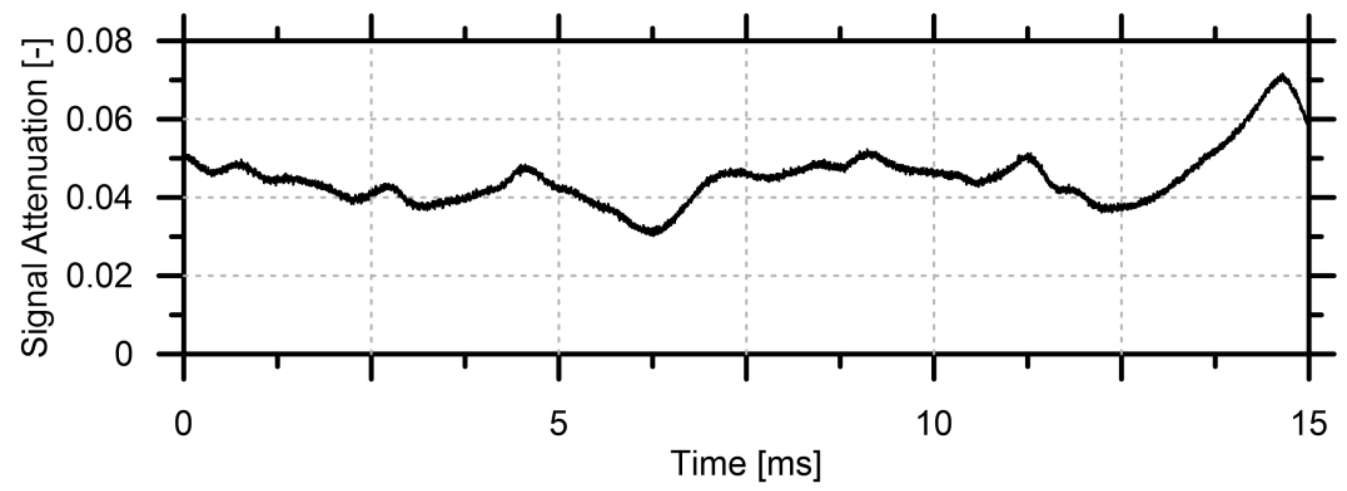

Figure 5.4: Time-resolved light soot attenuation signal over 15 milliseconds. During this time span, the $1428 \mathrm{~nm}$ laser $\left(\mathrm{H}_{2} \mathrm{O}\right.$ signal) completes 3 sweeps.

Within the $5 \mathrm{~ms}$ sweep period of the $\mathrm{H}_{2} \mathrm{O}$ laser at $1428 \mathrm{~nm}$, Figure 5.4 shows that soot may fluctuate by $20 \%$. Time-resolution and averaging effects were not considered during the primary simulation study presented in Chapter 3 . The presented simulations did not possess sufficient time-resolution and without other information available it was assumed that the $1428 \mathrm{~nm}$ laser would be able to sweep at a high enough rate. Potential effects of time-varying spectra were 
revisited using a simplified set of synthetic data to assess system performance in Appendix B. Results show that calculating a pseudo-time averaged $\mathrm{H}_{2} \mathrm{O}$ spectrum by averaging lead and lag sweeps (see Section 4.5.1) of the $1428 \mathrm{~nm}$ laser introduced a relative error where 95\% of measurements were within $\pm 5.13 \%$. Better-resolved assessments of time-varying spectral measurements is nevertheless a recommended area of future research. Importantly, the $200 \mathrm{~Hz}$ resolution is still a marked improvement from the capabilities of other flare plume measurement systems such as those by Weyant et al. (2016), Wood et al. (2012), and Wormhoudt et al. (2012), which had maximum sampling rates on the order of $1 \mathrm{~Hz}$. Moreover, it is anticipated that finer time-resolution in the present experiment would most likely indicate even broader variations in soot- $\mathrm{H}_{2} \mathrm{O}$ correlation (see Section 5.3) that might currently be obscured by time-averaging.

\subsection{Correlation Experiments}

Simultaneous measurements of path-averaged $\mathrm{H}_{2} \mathrm{O}$ and soot volume fractions were made for a range of fuels, fuel flow rates, and burner diameters as first outlined in Section 4.7. A range of fuels was selected to observe different sooting-propensities and to assess upper and lower limits of detection. At the lower limit, soot would not be detectable above the light attenuation from beam steering and system noise. At the upper limit uncertainties in soot optical properties would obscure the $\mathrm{H}_{2} \mathrm{O}$ absorption peaks in the isolated spectra.

Initial results suggested that ethylene and methane flares represented the upper and lower bounds of soot detection, respectively. The analysis conducted to reject methane and ethylene results is summarized in Appendix C. The 8 tests that remained were of flares of fuels characteristic of the Auca oil production region of Ecuador and the Bakken oil region of the United States. The optical measurement results of these fuel mixtures are discussed in this section. 
Of the 15-minute flare tests, roughly 4 minutes of measurements for each test were processed on a computer cluster over the course of 10 days, producing 18,000 individual pathaveraged results for each flare test. The Nelder-Mead optimization search to determine an effective column distribution that fitted measured $\mathrm{H}_{2} \mathrm{O}$ spectra was the most computationally intensive portion of the post-processing algorithm. This involved computing theoretical absorption spectra from HITRAN parameters for each new set of column distribution parameters until convergence criteria were met, typically taking 2 minutes per result. Processed results where the spectral fitting algorithm returned impossible column distribution parameters were removed.

Figure 5.5 shows a sample result of light attenuation for the two lasers from a Bakken flare. The length of the horizontal axis (2500 sample indices) corresponds to the $5 \mathrm{~ms}$ interval over which the lead/lag sweeps of the $1428 \mathrm{~nm}$ laser have been averaged. The time-resolved measurement from the $1654 \mathrm{~nm}$ laser shown in Figure 5.5b was similarly averaged to allow for mapping of soot attenuation onto the $1428 \mathrm{~nm}$ signal to isolate the $\mathrm{H}_{2} \mathrm{O}$ spectra.

(a)

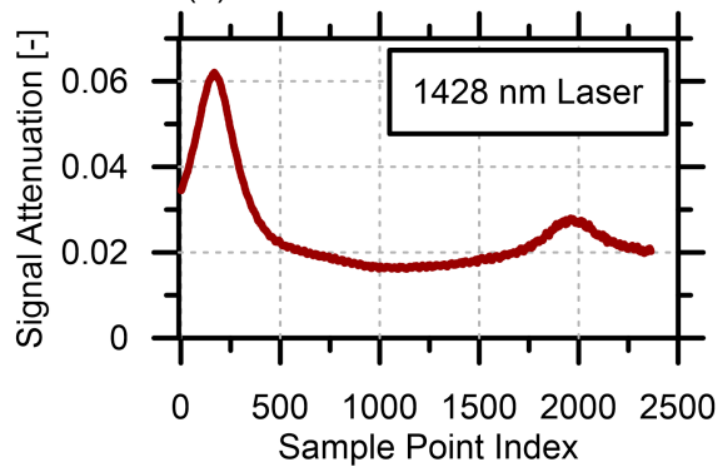

(b)

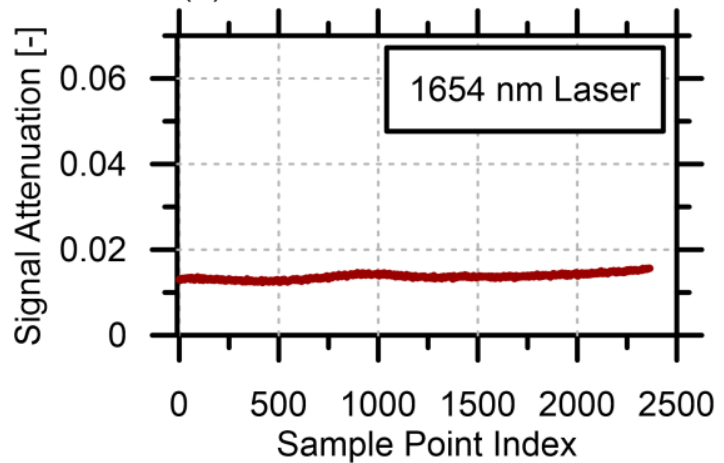

Figure 5.5: Sample $5 \mathrm{~ms}$ duration measurement of (a) $1428 \mathrm{~nm}\left(\mathrm{H}_{2} \mathrm{O}\right)$ and (b) $1654 \mathrm{~nm}$ (soot) laser signals through a Bakken flare plume. Time-resolved signals can be mapped to isolate spectral $\mathrm{H}_{2} \mathrm{O}$ absorption peaks from soot.

By using techniques developed in Chapter $2, \mathrm{H}_{2} \mathrm{O}$ spectra were isolated from soot attenuation. The isolated spectrum of Figure 5.5 is shown in Figure 5.6. The $\mathrm{H}_{2} \mathrm{O}$ spectra were displayed as a 
function of wavenumber by leveraging FRR and reference cell signals to map the scan of the laser's output wavenumber change to the sample index as discussed in Section 4.5.1. The isolated spectrum of Figure 5.6 is shown compared with the effective column distribution fit produced from the Nelder-Mead algorithm.

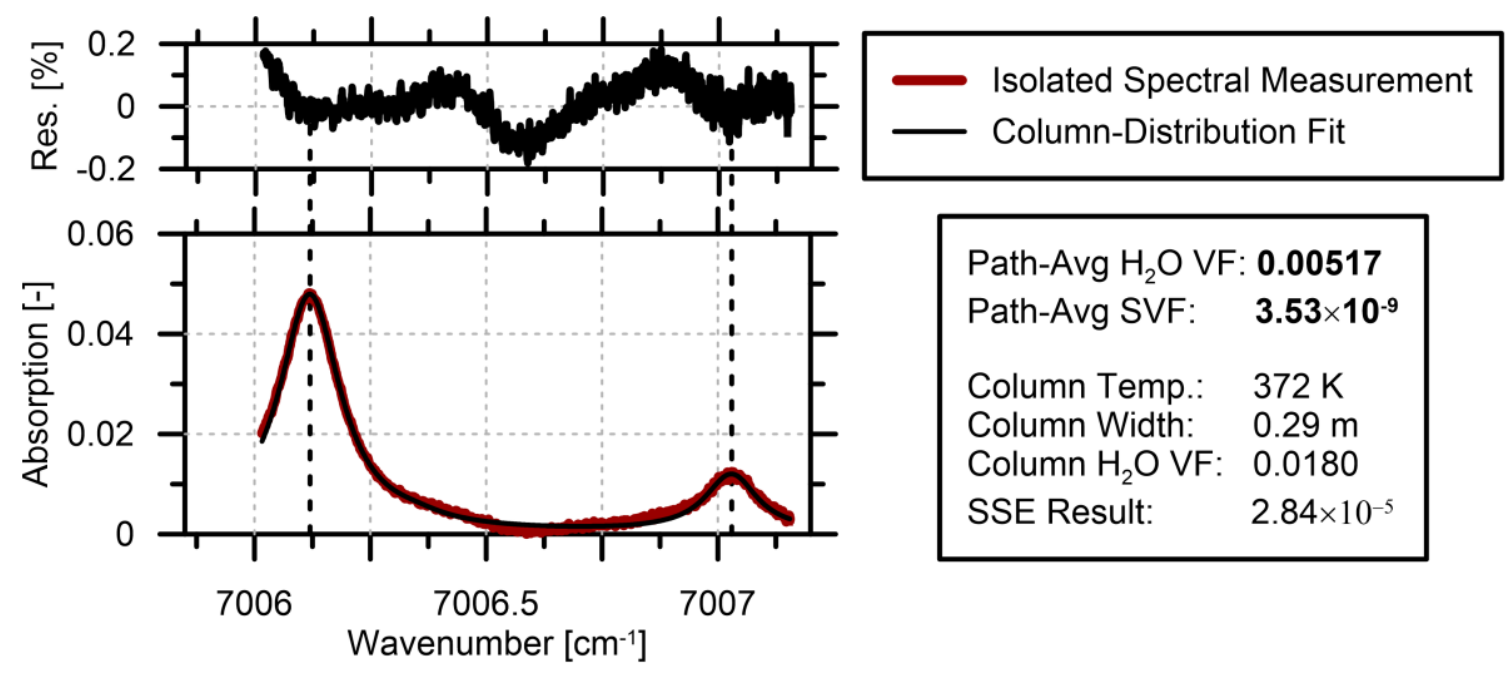

Figure 5.6: $\mathrm{H}_{2} \mathrm{O}$ absorption peaks in isolated, spectrally-resolved form compared to the column distribution result from the NM optimization search.

Before any additional analysis was performed, path-averaged temperatures were estimated from the column distribution results to evaluate the assumption that peak temperatures in the plume did not exceed $800 \mathrm{~K}$. From Section 3.3, path-averaged temperatures below $340 \mathrm{~K}$ generally represented measurements where peak temperatures were below $800 \mathrm{~K}$. If it can be assumed that the simulated plume is similar in structure to the plumes measured in the lab, results from pathaveraged temperature estimates should generally be below $340 \mathrm{~K}$. Figure 5.7 show histograms of estimated path-averaged temperatures for (a) Bakken, and (b) Ecuador fuel mixture tests. In the two tests shown, at least $96 \%$ of the individual measurements were below temperatures of $340 \mathrm{~K}$. This suggests that peak temperatures are likely within the expected range and do not far exceed $800 \mathrm{~K}$. 
(a)

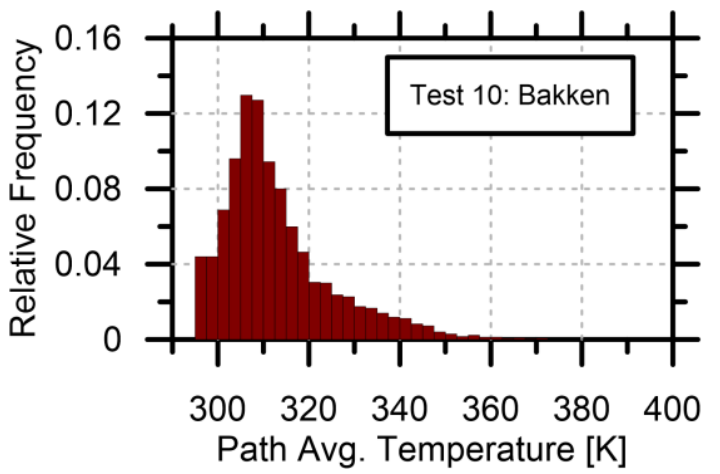

(b)

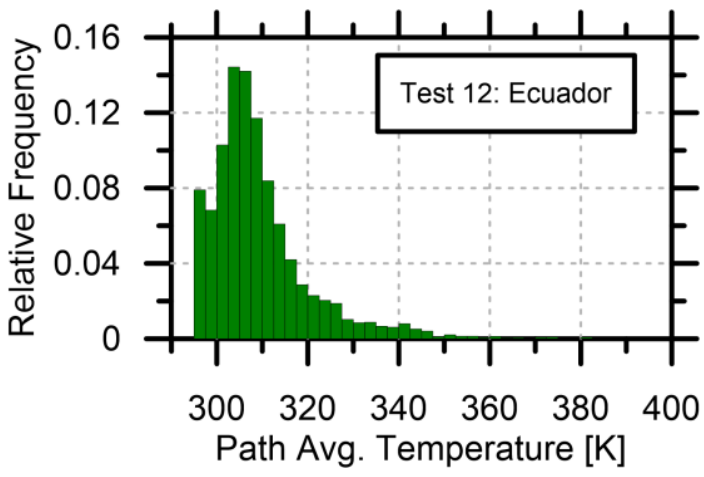

Figure 5.7: Histograms of estimated path-averaged temperatures in (a) Bakken, and (b) Ecuador fuel mixtures measurements.

With path-averaged temperatures confirmed to be within the expected range, the results from each test were then filtered to ensure only results of the best possible spectral fit were used to assess the species correlation. Appendix D discusses the considerations used to filter the data to produce conservative results of the species correlation. Only results of sum-of-squared-errors (SSE) below $10^{-4}$ were accepted; this represents the best possible fit between theoretical and measured spectra that was observed in the validation experiments of Section 4.6. Using the remaining data, scatter plots were constructed of the path-integrated soot and combustion-derived $\mathrm{H}_{2} \mathrm{O}$. A sample of these plots is shown in Figure 5.8. From these plots, there is a general correlation between the species, as should be expected since the production of $\mathrm{H}_{2} \mathrm{O}$ above ambient levels is necessary for the production of soot. However, instantaneous measurements show notable scatter about the central trend. 
(a)

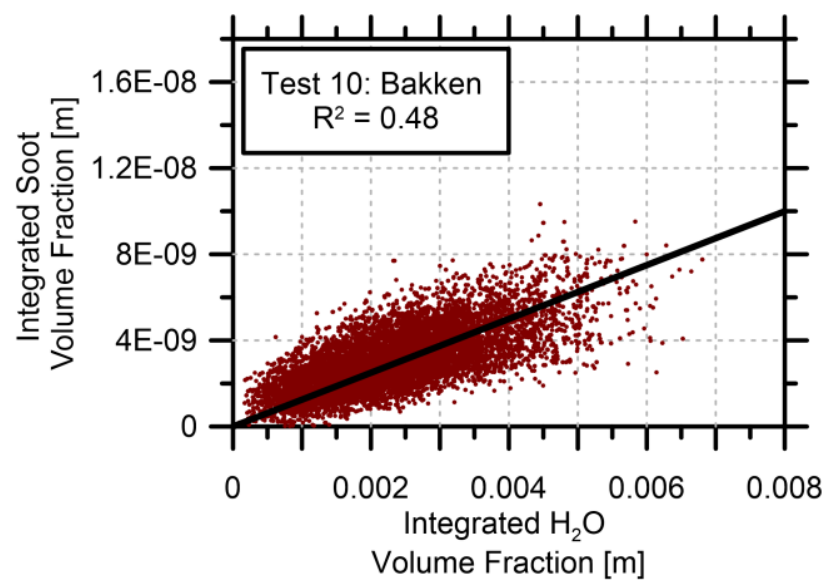

(b)

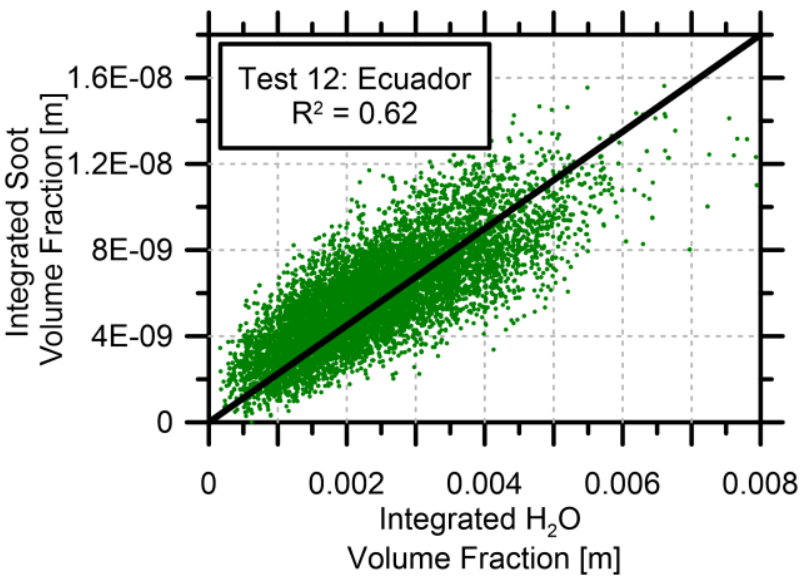

Figure 5.8: Scatter plots of soot and combustion-derived $\mathrm{H}_{2} \mathrm{O}$ path-integrated volume fraction for (a) Bakken, and (b) Ecuador fuel mixtures measurements with coefficients of determination as noted.

A more informative representation of the results was to determine the instantaneous ratio of soot to combustion-derived $\mathrm{H}_{2} \mathrm{O}$. Histograms of this soot- $\mathrm{H}_{2} \mathrm{O}$ ratio are shown in Figure 5.9 for the Bakken mixture flares and in Figure 5.10 for the Ecuador mixture tests. Relevant statistics for each test are summarized in Table 5.1. Table 5.1 also aggregates all results from each fuel type into two datasets and reports aggregated statistics. This is done to allow for a larger sample distribution for subsequent analysis. Data aggregation appeared reasonable since fuel flow rates and burner diameter changes did not significantly impact the soot- $\mathrm{H}_{2} \mathrm{O}$ distributions, at least in comparison to changing the fuel composition.
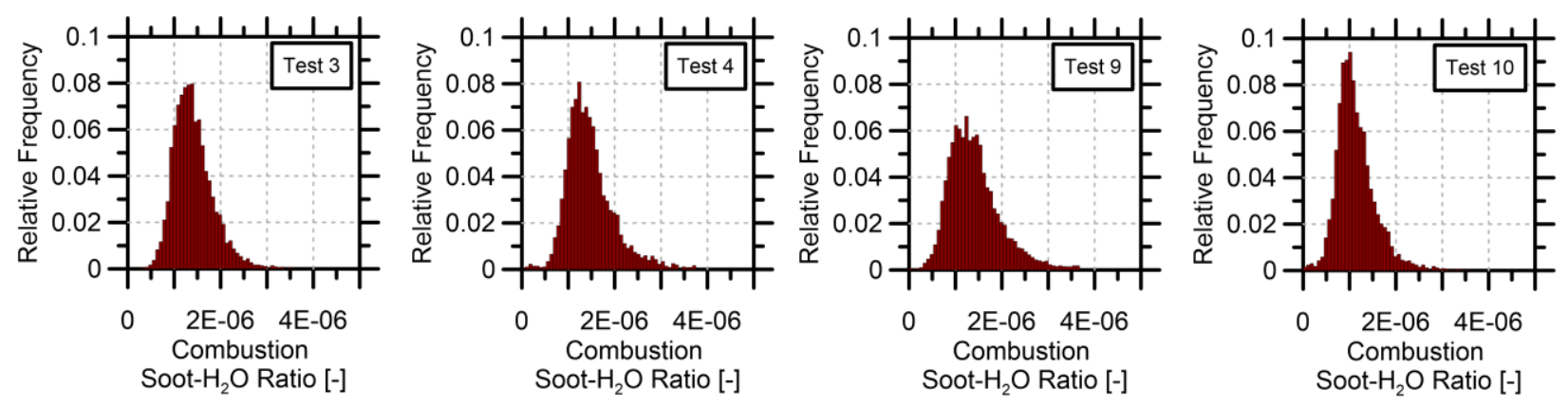

Figure 5.9: Soot-to- $\mathrm{H}_{2} \mathrm{O}$ volume fraction ratios for Bakken flares. 

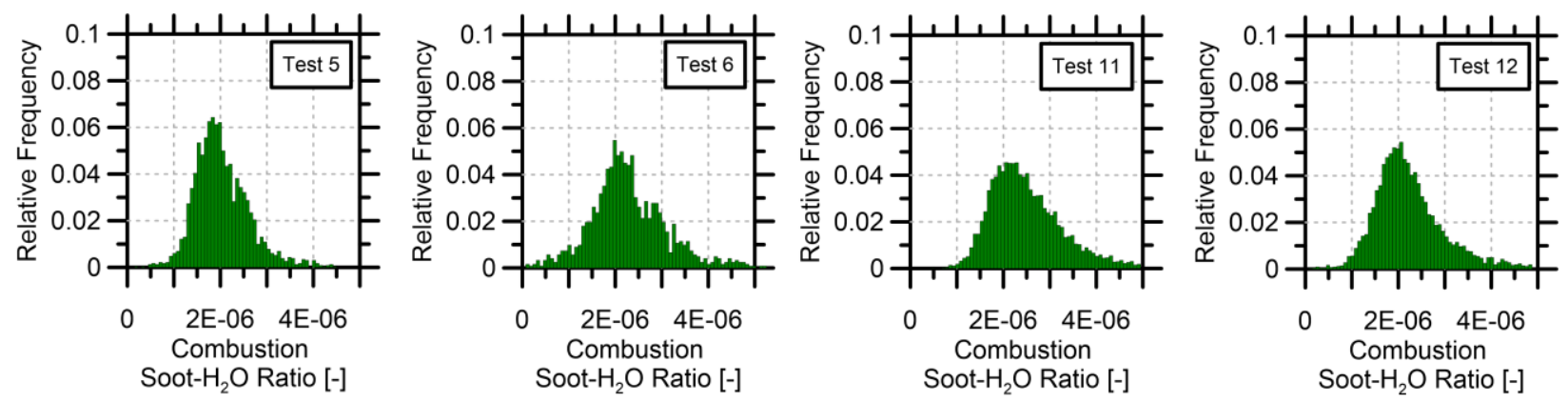

Figure 5.10: Soot-to-Combustion $\mathrm{H}_{2} \mathrm{O}$ volume fraction ratios for Ecuador flares.

The data in Table 5.1 show that all tests have a positive skew of soot- $\mathrm{H}_{2} \mathrm{O}$ ratio values. This suggests that short-lived bursts of high soot production uncorrelated with $\mathrm{H}_{2} \mathrm{O}$ exist. However, the skewness of these results must be compared to the inherent tendency of the measurement system to produce skewed soot- $\mathrm{H}_{2} \mathrm{O}$ ratio results. As shown in Appendix D, a ratio of two uncertain, non-negative quantities with distributions matching those of the current soot and $\mathrm{H}_{2} \mathrm{O}$ volume fraction uncertainties inherently produces a skewness of 0.37 . Nevertheless, the skewness of the Bakken and Ecuador results (2.85 and 2.53, respectively) are well in excess of this value.

Alternatively, if the estimated skewness of the measurement system from Appendix D is not well characterized, the skewness of the methane flare may be used as a more conservative estimate of the inherent measurement skew. The methane flare results possess very little soot but are otherwise subjected to the same measurement uncertainties. The methane results of soot $-\mathrm{H}_{2} \mathrm{O}$ skewness (included in Table 5.1) indicate skewness of 1.26 and 1.07 individually, and 0.98 when combined. Since all Bakken and Ecuador test results indicate skewness in excess of these values, some of this soot- $\mathrm{H}_{2} \mathrm{O}$ skewness must be intrinsic to the path-averaged species concentrations. Although it is difficult to quantify the skewness attributable to the true path-averaged soot- $\mathrm{H}_{2} \mathrm{O}$ volume fractions, this analysis shows the apparent existence of skewness. Furthermore, since the 
aggregated skewness values for the Bakken and Ecuador tests are fairly similar, it appears that this apparent skewness in the relative distribution of soot and $\mathrm{H}_{2} \mathrm{O}$ is not simply a scaling error with soot quantity. The similar skewness of the Ecuador and Bakken tests shows that although the soot quantities are changing, the skewness of the relative species concentrations is constant.

The positive skewness of the instantaneous soot- $\mathrm{H}_{2} \mathrm{O}$ ratio corresponding to short-lived bursts of high soot concentration is in line with the findings of Conrad \& Johnson (2017). Their study reported that total mass emission rates of soot from flares were heavily-weighted by short, localized bursts of high soot production giving distributions of instantaneous soot emission rates a positive skew. If this is indeed the case, extractive measurement systems such as the airplane studies by Gvakharia et al. (2017) and Weyant et al. (2016) which rely on a few extractive samples for flare performance estimations may yield unrepresentative results. By sampling from a positively skewed distribution of relative species concentrations, there is a high likelihood of underestimating the mean soot volume fractions. Quantitative implications for flare sampling systems, both extractive and remote, are discussed in the next section. The aggregated results of soot- $\mathrm{H}_{2} \mathrm{O}$ ratios for the two fuel types are shown in Figure 5.11 along with their cumulative distribution functions; both exhibit positive skew in excess of 2.5. 
Table 5.1: Summary of Flare Test Soot- $\mathrm{H}_{2} \mathrm{O}$ Ratio Results and Relevant Statistics

\begin{tabular}{|c|c|c|c|c|c|c|}
\hline Test No. & Fuel Type & Test Condition & Points & $\begin{array}{l}\text { Mean Soot- } \\
\mathrm{H}_{2} \mathrm{O} \text { Ratio }\end{array}$ & $\begin{array}{c}\text { Soot- } \mathrm{H}_{2} \mathrm{O} \text { Std. } \\
\text { Dev. }\end{array}$ & Skewness \\
\hline 1 & \multirow{2}{*}{ Methane } & $40 \mathrm{SLPM}, 25.4 \mathrm{~mm}$ & 2463 & $1.16 \mathrm{E}-07$ & $8.90 \mathrm{e}-08$ & 1.26 \\
\hline 2 & & $60 \mathrm{SLPM}, 25.4 \mathrm{~mm}$ & 7556 & $-1.24 \mathrm{E}-08^{\mathrm{a}}$ & $7.85 \mathrm{e}-08$ & 1.07 \\
\hline 3 & \multirow{4}{*}{ Bakken } & 40 SLPM, $25.4 \mathrm{~mm}$ & 6351 & $1.42 \mathrm{E}-06$ & 4.57E-07 & 1.68 \\
\hline 4 & & 60 SLPM, $25.4 \mathrm{~mm}$ & 3541 & $1.49 \mathrm{E}-06$ & $6.59 \mathrm{E}-07$ & 2.84 \\
\hline 9 & & 40 SLPM, $76.2 \mathrm{~mm}$ & 10646 & $1.45 \mathrm{E}-06$ & $6.94 \mathrm{E}-07$ & 3.00 \\
\hline 10 & & $60 \mathrm{SLPM}, 76.2 \mathrm{~mm}$ & 3300 & $1.15 \mathrm{E}-06$ & $5.06 \mathrm{E}-07$ & 3.46 \\
\hline 5 & \multirow{4}{*}{ Ecuador } & $40 \mathrm{SLPM}, 25.4 \mathrm{~mm}$ & 2316 & $2.05 \mathrm{E}-06$ & $6.43 \mathrm{E}-07$ & 1.63 \\
\hline 6 & & $60 \mathrm{SLPM}, 25.4 \mathrm{~mm}$ & 1258 & $2.35 \mathrm{E}-06$ & $1.04 \mathrm{E}-06$ & 1.65 \\
\hline 11 & & $40 \mathrm{SLPM}, 76.2 \mathrm{~mm}$ & 10027 & $2.58 \mathrm{E}-06$ & 9.84E-07 & 2.27 \\
\hline 12 & & $60 \mathrm{SLPM}, 76.2 \mathrm{~mm}$ & 4359 & $2.34 \mathrm{E}-06$ & $1.04 \mathrm{E}-06$ & 3.48 \\
\hline \multirow{3}{*}{ Aggregated } & Methane & \multirow{3}{*}{ Various } & 10,019 & $1.92 \mathrm{E}-08$ & $9.83 \mathrm{E}-07$ & 0.98 \\
\hline & Bakken & & 23,868 & 1.41E-06 & $5.94 \mathrm{E}-07$ & 2.85 \\
\hline & Ecuador & & 17,960 & 2.44E-06 & 9.83E-07 & 2.53 \\
\hline
\end{tabular}

a This non-physical mean value of the soot- $\mathrm{H}_{2} \mathrm{O}$ ratio is caused by beam steering which may cause light to be gained by the optical system in some cases. This was discussed in Section 4.9.

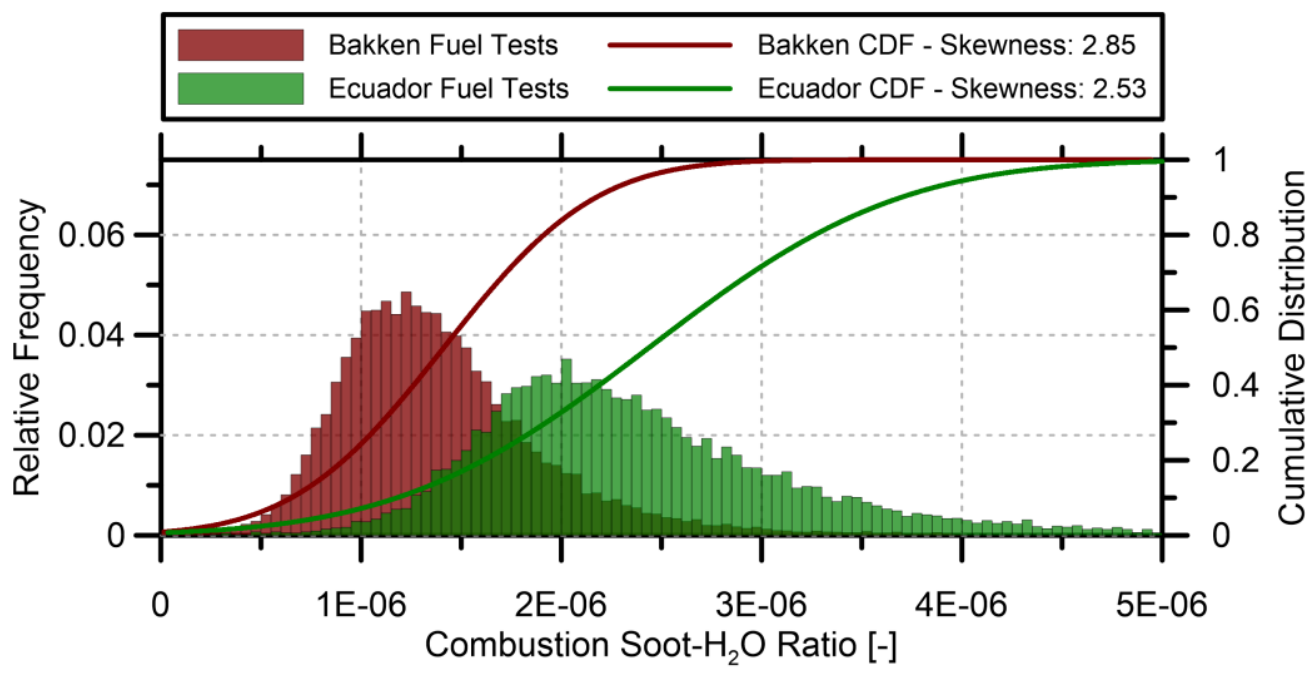

Figure 5.11: Combined Soot- $\mathrm{H}_{2} \mathrm{O}$ Ratios for the two fuel types considered along with their respective cumulative distribution functions (CDFs).

Although not the focus of the study, mean values of soot- $\mathrm{H}_{2} \mathrm{O}$ ratios from the two aggregated datasets were used to estimate equivalent emission factors of soot to show that values are within a reasonable range of emission factors from other measurement techniques. Using a simple stoichiometric relationship, these mean soot- $\mathrm{H}_{2} \mathrm{O}$ ratios suggest black carbon emission factors of 7.4 and $14.3 \mathrm{~g} / \mathrm{m}^{3}$ for the Bakken and Ecuador fuels, respectively. In comparison, the relationship of black carbon emission factor to $\mathrm{HHV}_{\mathrm{v}}$ from sky-LOSA studies would suggest emission factors 
of 2.7 and $3.9 \mathrm{~g} / \mathrm{m}^{3}$ (Conrad \& Johnson, 2017); measurements from this thesis suggest an increase of $95 \%$ of emission factors between the fuel types while sky-LOSA would predict $44 \%$. Although this system was not intended to make absolute measurements of soot emission factors, the apparent emission factors are still within a factor of four to values anticipated through sky-LOSA measurements. Being within an order of magnitude of published emission factor results lends confidence to the measurement techniques considering the soot optical properties were estimated and the $\mathrm{H}_{2} \mathrm{O}$ spectral measurement was uncalibrated. Apparent emission factors are much more in line with sky-LOSA emissions factor findings than with the study by Weyant et al. (2016) which reported emission factor estimates for Bakken fuel flares to be at most $0.43 \mathrm{~g} / \mathrm{m}^{3}$.

\subsection{Implications for Flare Sampling Techniques}

To illustrate the effects that fluctuations in relative species concentrations can have on a measurement system, the aggregated results from Figure 5.11 were assumed to be true pathaveraged distributions of soot- $\mathrm{H}_{2} \mathrm{O}$ ratios occurring in flare plumes for the Bakken and Ecuador fuel mixtures. Suppose a measurement system of interest (e.g., an airplane flying plume transects or a short duration spectral measurement recorded from the ground) can only obtain $N$ distinct path-averaged measurements. The computed mean of these $N$ samples may not yield a value sufficiently close to the true mean. By taking the data from Figure 5.11 and performing 10,000 iterations of taking $N$-sized sample sets allowing replacement and computing a sample mean, distributions of sample mean values are plotted in Figure 5.12 and Figure 5.13 using the Bakken and Ecuador data, respectively. 


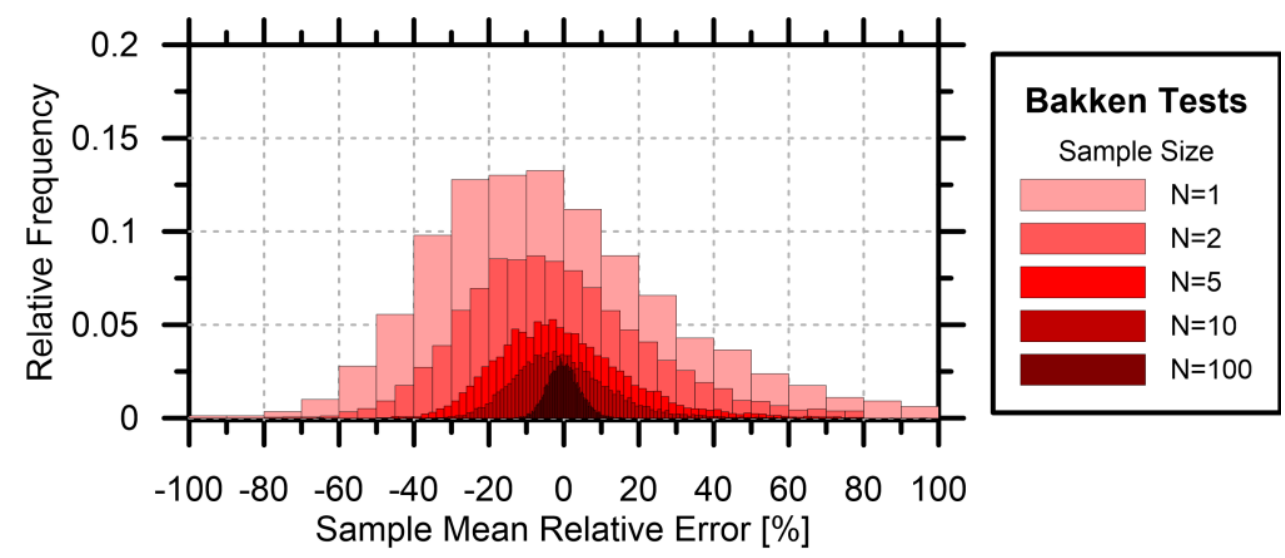

Figure 5.12: Distributions of sample mean for sample sets of size $\mathrm{N}$ from $\mathrm{Soot}-\mathrm{H}_{2} \mathrm{O}$ ratio results of the Bakken flare tests.

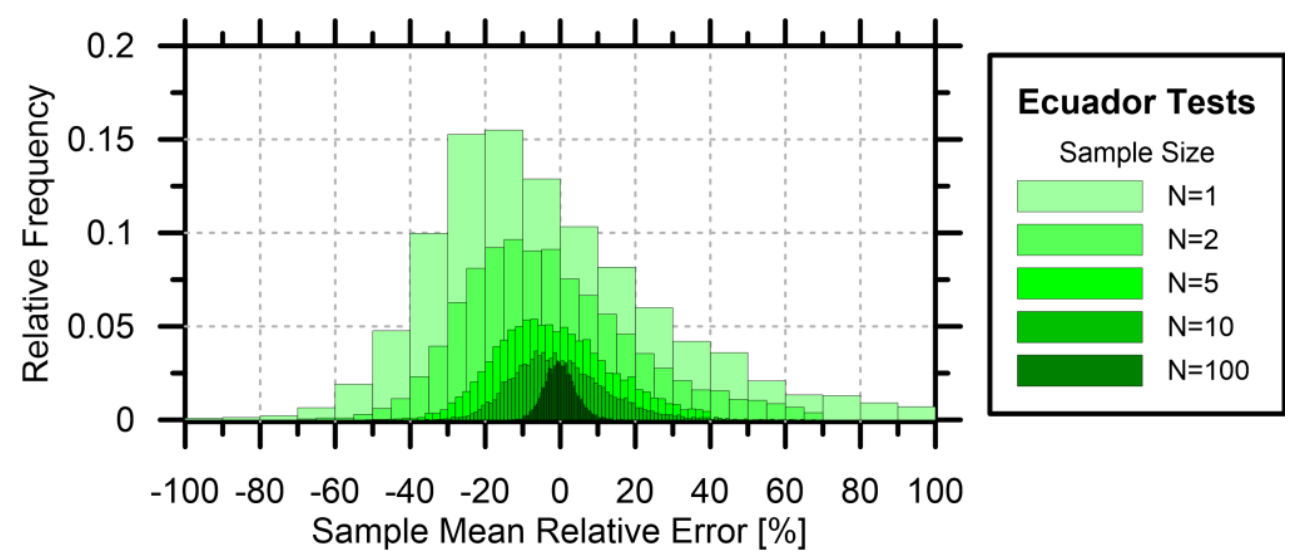

Figure 5.13: Distributions of sample mean for sample sets of size $N$ from $\mathrm{Soot}-\mathrm{H}_{2} \mathrm{O}$ ratio results of the Ecuador flare tests.

If a sample size of 1 is taken on this path-averaged optical measurement system, it is possible to obtain values within a range of $\pm 100 \%$ about the true mean value. This implies that measurements relying on well-correlated species concentrations may yield results anywhere between zero and double the true mean. Observing the distributions of samples mean for $N=1$, there is an apparent skew towards sample means below the true mean. In other words, there is an increased likelihood that when sampling from the distributions of Figure 5.11, estimated mean values will underestimate the true mean. In the 10,000 -iteration samples where $N=1,58$ and $60 \%$ of the sample mean values fell below the true mean value for the Bakken and Ecuador fuel mixtures, 
respectively. For $N=5$, this proportion drops to $\sim 55 \%$ for both datasets; proportions approach $50 \%$ as $N$ is increased.

If the sample size is increased to 10 path-averaged measurements, the sample mean is likely within $\pm 30 \%$ from the true mean. In general, as the sample size is increased the relative error of the sample mean will decrease and the skewness approaches zero meaning a reduced likelihood of underestimating the true mean. This trend is shown in Figure 5.14 for sample sizes up to 10,000.
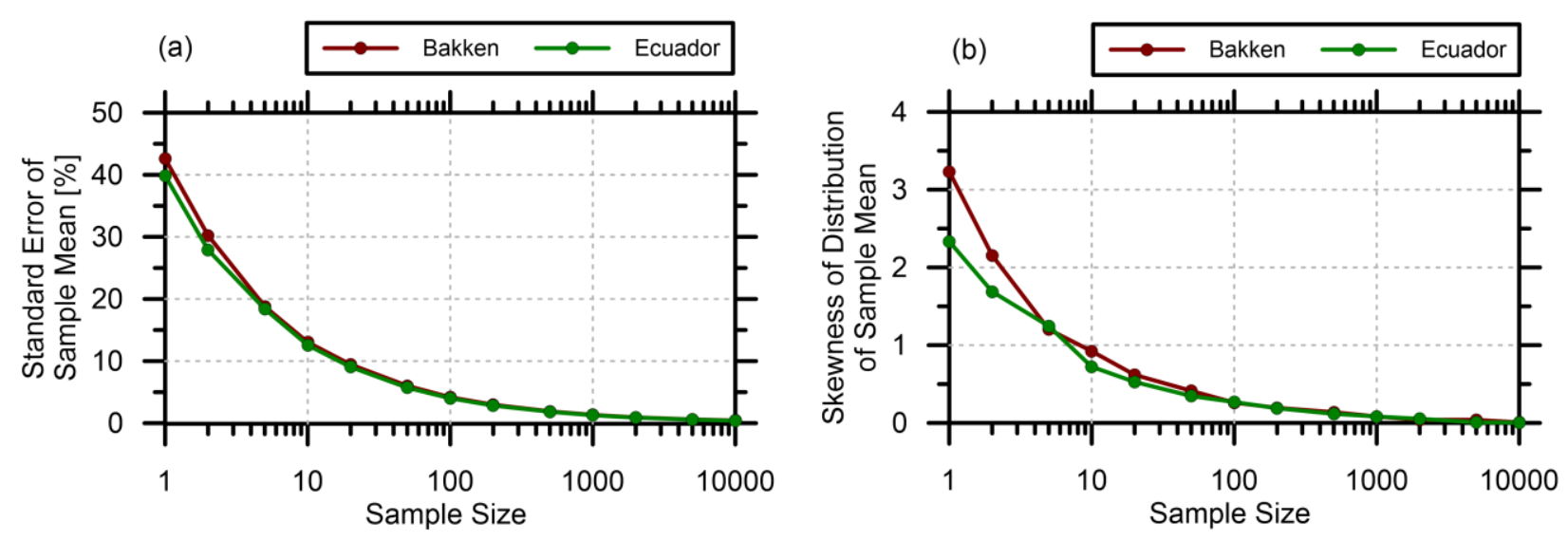

Figure 5.14: (a) Standard error of the sample mean and (b) skew of the distribution with increased sample size. This indicates the convergence toward the population mean with increased sample size.

Clearly, it is advisable to perform as many measurements as feasible to obtain the best estimates of flare performance for any technique. However, this is not easy with many techniques. For extractive sampling using aircraft, such as in the studies by Gvakharia et al. (2017) and Weyant et al. (2016), it would be impractical to perform 1000 plume transects on individual flares. Having some understanding of the spatial and temporal correlation of species in the plume would allow for accurate estimates of uncertainty to be produced for the aircraft measurements that might only perform 7 plume transects.

Weyant et al. (2016) applied their mean emission factor of $0.43 \mathrm{~g} / \mathrm{m}^{3}$ from aircraft measurements of flares in the Bakken region to an assumed global annual flared volume of 150 
billion $\mathrm{m}^{3}$. This suggested annual black carbon (BC) emission of $20 \mathrm{Gg}$ from flaring, which is substantially lower than estimates of annual BC of $228 \mathrm{Gg}$ reported by Stohl et al. (2013). However, if it is assumed that the emission factor from Weyant et al. was susceptible to variations in local species correlation and took two transect measurements (i.e., $N=2$ ), the reported value may be $\pm 60 \%$ from the true value from Figure 5.12 (i.e., the true emission factor could lie anywhere between 0.27 and $1.08 \mathrm{~g} / \mathrm{m}^{3}$ ). Unfortunately this does not fully explain findings by Weyant et al. (2016) where their measurements on the same flares over different days yielded variations of up to 12 times. However, using the 0.27 and $1.08 \mathrm{~g} / \mathrm{m}^{3}$ values would suggest the global $\mathrm{BC}$ emission from flaring could be between 41 and $162 \mathrm{Gg}$ (where Weyant et al. would suggest $65 \mathrm{Gg}$ ). The upper bound of this range is much closer to the estimates of $228 \mathrm{Gg}$ by Stohl et al. (2013) and may partially explain the discrepancy.

The measurement technique using aircrafts is by no means the only technique susceptible to localized variations in relative species concentration. The use of drones for environmental monitoring has proliferated in recent years especially in the oil and gas industry (Satterlee, 2016). Drones studies such as the one conducted by Krause \& Leirvik (2018) to measure black carbon emission factors may have an even higher potential to be affected by local fluctuations in relative species concentrations. These studies observe much more spatially-resolved flare exhaust than the measurement technique presented in this thesis meaning fluctuations in relative species concentrations would not be smoothed out over a long path. Without a firm understanding of the nature of these fluctuations drone measurements may produce even less representative results than their path-averaged counterparts.

Although uncertainties in individual flare performance measurements may not always be substantially affected by fluctuations in species correlation, there is still a potential for these 
uncertainties to be inflated in an area of study where a wealth of measurements is lacking. Since flare performance measurements are not currently performed on a routine basis, performance measurements from only a select few studies are often used to extrapolate global implications. This is best exemplified by the study by McDaniel (1983). The report by McDaniel used singlepoint measurements of a flare of crude propylene to determine a black carbon emission factor. This single-fuel, point-measurement study forms the basis of a significant portion of black carbon inventories in both the United States and Canada. Uncertainties in global pollutant emission inventories which stem from uncertainties in the few available measurements can have significant impact on climate modeling studies which strive to determine the most effective pollution mitigation strategies (Winiger et al., 2019).

Issues of local species correlation may also extend to other pollutant emissions. If spatial and temporal variations in soot- $\mathrm{H}_{2} \mathrm{O}$ exist in flare plumes, it is likely that fluctuations in local combustion performance could also produce variations affecting other flare performance metrics. This might include measurements for other pollutant emission factors (e.g., $\left.\mathrm{H}_{2} \mathrm{~S}, \mathrm{SO}_{2}, \mathrm{CO}\right)$ as well as measurements of flare efficiency and uncombusted fuels escaping the flare.

The variation in soot- $\mathrm{H}_{2} \mathrm{O}$ ratio that appears in the path-averaged measurements in this thesis imply the existence of short, localized bursts of high soot production that are not wellcorrelated with other combustion products. This phenomenon must be characterized in terms of time- and length-scale to be able to determine its effects on the different flare performance measurements techniques that currently exist. Characterization of these high-soot flow structures will allow for the development of a measurement protocol which could quantify measurement uncertainty and/or recommend minimum sampling times to reduce uncertainty. 


\section{Chapter 6 Conclusion}

A spectroscopic measurement system was developed to measure path-averaged volume fractions of $\mathrm{H}_{2} \mathrm{O}$ and soot in turbulent flare plumes. The system relied on tunable diode laser absorption spectroscopy (TDLAS) and line-of-sight attenuation (LOSA) techniques to measure $\mathrm{H}_{2} \mathrm{O}$ and soot, respectively. At the measurement wavelengths of 1428 and $1654 \mathrm{~nm}$, narrow spectral absorption of $\mathrm{H}_{2} \mathrm{O}$ and broadband light attenuation due to soot can be measured and used to yield pathaveraged species volume fractions. The optical techniques were tailored to measure these species in a non-uniform flow-field and $\mathrm{H}_{2} \mathrm{O}$ absorption peaks were selected to ensure temperature and volume fraction fluctuations could be distinguished in the path-averaged measurement.

The performance of the optical techniques was first evaluated using theoretical signals generated from large eddy simulation (LES) data of methane flares in crossflow. The results of instantaneous path-averaging showed relative errors were on average $-0.25 \%$ with $95 \%$ of measurements being within $\pm 6.74 \%$. Soot volume fraction measurements in simulation were estimated to within $0.2 \mathrm{ppb}$ on path-averaged signals ranging from 0 to $15 \mathrm{ppb}$; the error was due solely to Gaussian noise.

The designed and tested optical system was capable of measuring $\mathrm{H}_{2} \mathrm{O}$ absorption peaks at a rate of $200 \mathrm{~Hz}$, which was limited by the spectral tuning characteristics of the distributed feedback laser. All open path and reference detector signals were streamed to a remote acquisition and control station at $1 \mathrm{MHz}$ per channel. Reference optics were housed in a custom-made thermally-stable enclosure to minimize measurement drift. Preliminary experiments on a flare produced at the Carleton University Flare Facility (CUFF) measured the soot extinction coefficient ratio between selected the wavelengths, which was necessary to isolate $\mathrm{H}_{2} \mathrm{O}$ absorption spectra 
from broadband soot attenuation in subsequent measurements. The soot extinction coefficient ratio was estimated to be $0.964 \pm 0.013$, which agreed closely with soot extinction coefficient ratios calculated from extinction measurements in the literature. The soot optical property measurement data were also used to infer effective timescales of the path-averaged measurement. This demonstrated that soot attenuation fluctuations in the turbulent plume were detectable at the submillisecond scale; this timescale implied that the $200 \mathrm{~Hz}(5 \mathrm{~ms}$ period) sweeps of the $1428 \mathrm{~nm}$ $\left(\mathrm{H}_{2} \mathrm{O}\right)$ laser would induce time-averaging and potentially average out more minute fluctuations in relative species concentrations. Time-averaging was shown in simplified simulations to produce relative error in path-averaged $\mathrm{H}_{2} \mathrm{O}$ estimates with mean of $0.28 \%$ where $95 \%$ of measurements were within $\pm 5.13 \%$. The effects of beam steering on inferred measurements was also assessed. Light loss due to beam steering is necessarily interpreted as increased presence of soot along the optical path. Beam steering measurements during hydrogen flare tests showed inferred soot volume fractions with a standard deviation of $1.61 \times 10^{-10}$; for comparison, the median soot volume fraction of the lower sooting Bakken fuel mixture was $2.5 \times 10^{-9}$.

The developed system was then used to measure path-averaged species volume fractions in lab-scale turbulent plumes at the Carleton University Flare Facility (CUFF) for multiple fuel mixtures, burner diameters, and fuel flow rates. The species volume fractions were used to inspect path-averaged species correlations. The results showed a positive skew in the distribution of soot$\mathrm{H}_{2} \mathrm{O}$ ratios in all cases. This positive skew was above inherent skewness arising from the ratio of uncertain measured values from the measurement system and implies the existence of short, localized bursts of high soot production that are not correlated with $\mathrm{H}_{2} \mathrm{O}$. This finding supports the observations of Conrad \& Johnson (2017) who reported that mass emission rates of black carbon from flares were dominated by short bursts of high soot emission. 
Critically, the skewness of soot- $\mathrm{H}_{2} \mathrm{O}$ ratios in the path-averaged measurement would tend to cause most flare measurement techniques to underestimate soot emission factors due to the reduced likelihood of measuring the short-lived, highly-sooting bursts in the plume. Statistical analysis based on the present results was performed which demonstrates the importance of sufficient samples to yield representative mean performance values. Although this thesis shows the apparent existence of localized bursts of high soot production uncorrelated with other combustion species, further study is necessary to quantify the time- and length-scales of these flow structures. This understanding would enable development of unified measurement protocols more accurate characterization of uncertainty bounds for different measurement techniques.

\subsection{Future Considerations}

The time-resolution of the path-averaged measurements was limited by the maximum currenttuning ability of the $1428 \mathrm{~nm} \mathrm{H}_{2} \mathrm{O}$ detection laser. The $200 \mathrm{~Hz}$ maximum sweep rate was shown to be insufficient to resolve sub-millisecond fluctuations in soot and $\mathrm{H}_{2} \mathrm{O}$ volume fractions. The time-resolution of the designed optical system would be improved by using a laser capable of measuring $\mathrm{H}_{2} \mathrm{O}$ absorption peaks at a higher rate. This could be achieved either by the selection of new $\mathrm{H}_{2} \mathrm{O}$ absorption peaks that are nearer in wavelength and can be swept across more rapidly; alternatively, more than one distributed feedback laser could be leveraged to reduce the sweep amplitude thereby allowing for increased sweep rates.

Detection capabilities could also be improved by further reducing light loss due to beam steering which introduced error in soot volume fraction and would tend to introduce biasing of the isolation $\mathrm{H}_{2} \mathrm{O}$ absorption spectra. If the system was desired to provide more quantitative estimates of the soot- $\mathrm{H}_{2} \mathrm{O}$ ratio, absolute soot optical properties would be required and the $\mathrm{H}_{2} \mathrm{O}$ spectral 
parameters from HITRAN would need to be measured in controlled experiments to verify their accuracy.

Towards the quantification of the length- and time-scales of the relative species concentrations in turbulent flare plumes, improved spatial and temporal resolution would be necessary. Improved spatial resolution could likely be achieved by miniaturizing the system presented in this thesis and mounting it on a drone. The drone would be able to measure more spatially resolved species correlations in flare plumes in the field. At this reduced path length, it would also be possible to switch to $\mathrm{CO}_{2}$ detection which has a reduced ambient contribution to the measurement in comparison to $\mathrm{H}_{2} \mathrm{O}$ where ambient volume fractions needed to be removed from the measured results. Detection of $\mathrm{CO}_{2}$ would also allow for carbon-balances to be performed directly to assess emission factors. 


\section{References}

Blackwood, T. R. (2000). An evaluation of flare combustion efficiency using open-path Fourier transform infrared technology. Journal of the Air \& Waste Management Association, 50(10), $1714-1722$.

Blokh, A. G. (1988). Heat Transfer In Steam Boiler Furnaces (1st ed.). CRC Press.

Bond, T. C., Doherty, S. J., Fahey, D. W., Forster, P. M., Berntsen, T. K., DeAngelo, B. J., ... Zender, C. S. (2013). Bounding the role of black carbon in the climate system: A scientific assessment. Journal of Geophysical Research: Atmospheres, 118(11), 5380-5552. https://doi.org/10.1002/jgrd.50171

Brandt, A. R., Yeskoo, T., McNally, M. S., Vafi, K., Yeh, S., Cai, H., \& Wang, M. Q. (2016). Energy Intensity and Greenhouse Gas Emissions from Tight Oil Production in the Bakken Formation. Energy \& Fuels, 30(11), 9613-9621. https://doi.org/10.1021/acs.energyfuels.6b01907

Cape, J. N., Coyle, M., \& Dumitrean, P. (2012). The atmospheric lifetime of black carbon. Atmospheric Environment, 59, 256-263. https://doi.org/10.1016/j.atmosenv.2012.05.030

Chang, H., \& Charalampopoulos, T. T. (1990). Determination of the Wavelength Dependence of Refractive Indices of Flame Soot. Proceedings of the Royal Society A: Mathematical, Physical and Engineering Sciences, 430(1880), 577-591. https://doi.org/10.1098/rspa.1990.0107

Coderre, A. R., Thomson, K. A., Snelling, D. R., \& Johnson, M. R. (2011). Spectrally-Resolved Light Absorption Properties of Cooled Soot from a Methane Flame. Applied Physics B, 104(1), 175-188. https://doi.org/10.1007/s00340-011-4448-9

Conrad, B. M., \& Johnson, M. R. (2017). Field Measurements of Black Carbon Yields from Gas Flaring. Environmental Science \& Technology, 51(3), 1893-1900. https://doi.org/10.1021/acs.est.6b03690

Conrad, B. M., \& Johnson, M. R. (2019). Mass Absorption Cross-Section of Flare-Generated Black Carbon: Variability, Predictive Model, and Implications. Submitted to Carbon.

D’Amato, F. (2006). Laser Spectrometers for Atmospheric Analysis. (B. D. Bartolo \& O. Forte, Eds.), Spectroscopy. Springer.

Demtröder, W. (2014). Laser Spectroscopy 1 (5th ed.). Springer Berlin Heidelberg.

Elvidge, C. D., Zhizhin, M., Baugh, K., Hsu, F., \& Ghosh, T. (2015). Methods for Global Survey of Natural Gas Flaring from Visible Infrared Imaging Radiometer Suite Data. Energies, 9(1), 14. https://doi.org/10.3390/en9010014

Farooq, A., Jeffries, J. B., \& Hanson, R. K. (2008). In situ combustion measurements of H2O and temperature near $2.5 \mu \mathrm{m}$ using tunable diode laser absorption. Measurement Science and 
Technology, 19(7), 075604. https://doi.org/10.1088/0957-0233/19/7/075604

Fischer, J. (2003). Total internal partition sums for molecular species in the 2000 edition of the HITRAN database. Journal of Quantitative Spectroscopy and Radiative Transfer, 82(1-4), 401-412. https://doi.org/10.1016/S0022-4073(03)00166-3

Flanner, M. G. (2013). Arctic climate sensitivity to local black carbon. Journal of Geophysical Research Atmospheres, 118(4), 1840-1851. https://doi.org/10.1002/jgrd.50176

Fotia, M. L., Sell, B. C., Hoke, J., Wakefield, S., \& Schauer, F. (2015). 1 kHz Mid-IR Absorption Spectroscopy for CO and CO 2 Concentration and Temperature Measurement. Combustion Science and Technology, 187(12), 1922-1936. https://doi.org/10.1080/00102202.2015.1069281

Gasser, T., Peters, G. P., Fuglestvedt, J. S., Collins, W. J., Shindell, D. T., \& Ciais, P. (2017). Accounting for the climate- carbon feedback in emission metrics. Earth System Dynamics, 8(2), 235-253. https://doi.org/10.5194/esd-8-235-2017

Gordley, L. L., Marshall, B. T., \& Allen Chu, D. (1994). Linepak: Algorithms for modeling spectral transmittance and radiance. Journal of Quantitative Spectroscopy and Radiative Transfer. https://doi.org/10.1016/0022-4073(94)90025-6

Gvakharia, A., Kort, E. A., Brandt, A. R., Peischl, J., Ryerson, T. B., Schwarz, J. P., ... Sweeney, C. (2017). Methane, Black Carbon, and Ethane Emissions from Natural Gas Flares in the Bakken Shale, North Dakota. Environmental Science and Technology, 51(9), 5317-5325. https://doi.org/10.1021/acs.est.6b05183

Hanson, R. K., Spearrin, R. M., \& Goldenstein, C. S. (2016). Spectroscopy and Optical Diagnostics for Gases (1st ed.). Springer.

Herndon, S. C., Nelson, D. D., Wood, E. C., Knighton, W. B., Kolb, C. E., Kodesh, Z., ... Allen, D. T. (2012). Application of the Carbon Balance Method to Flare Emissions Characteristics. Industrial \& Engineering Chemistry Research, 51(39), 12577-12585. https://doi.org/10.1021/ie202676b

Howell, J. R., Siegel, R., \& Mengüç, M. P. (2016). Thermal Radiation Heat Transfer (6th ed.). Boca Raton, FL: Taylor \& Francis.

Jacobson, M. Z. (2010). Short-term effects of controlling fossil-fuel soot, biofuel soot and gases, and methane on climate, Arctic ice, and air pollution health. Journal of Geophysical Research, 115(D14209), 1-24. https://doi.org/10.1029/2009JD013795

Johnson, L. A. (2006, February). Laser Diode Burn-in and Reliability Testing. IEEE Communiations.

Johnson, M. R., Devillers, R. W., \& Thomson, K. A. (2011). Quantitative Field Measurement of Soot Emission from a Large Gas Flare Using Sky-LOSA. Environmental Science \& Technology, 45(1), 345-350. https://doi.org/10.1021/es102230y

Johnson, M. R., Devillers, R. W., \& Thomson, K. A. (2013). A Generalized Sky-LOSA Method 
to Quantify Soot/Black Carbon Emission Rates in Atmospheric Plumes of Gas Flares. Aerosol Science and Technology, 47(9), 1017-1029. https://doi.org/10.1080/02786826.2013.809401

Knighton, W. B., Herndon, S. C., Franklin, J. F., Wood, E. C., Wormhoudt, J., Brooks, W., ... Allen, D. T. (2012). Direct measurement of volatile organic compound emissions from industrial flares using real-time online techniques: Proton Transfer Reaction Mass Spectrometry and Tunable Infrared Laser Differential Absorption Spectroscopy. Industrial \& Engineering Chemistry Research, 51(39), 12674-12684. https://doi.org/10.1021/ie202695v

Köylü, Ü. Ö., \& Faeth, G. M. (1994). Optical properties of overfire soot in buoyant turbulent diffusion flames at long residence times. Journal of Heat Transfer, 116(1), 152-159. https://doi.org/10.1115/1.2910849

Krause, D. F., \& Leirvik, F. (2018). Field Measurement of BC Emissions from Rolvsnes Well Test Flare.

Krishnan, S. S., Lin, K.-C., \& Faeth, G. M. (2001). Extinction and Scattering Properties of Soot Emitted From Buoyant Turbulent Diffusion Flames. Journal of Heat Transfer, 123(2), 331. https://doi.org/10.1115/1.1350823

Liu, C., Xu, L., \& Cao, Z. (2013). Measurement of nonuniform temperature and concentration distributions by combining line-of-sight tunable diode laser absorption spectroscopy with regularization methods. Applied Optics, 52(20), 4827. https://doi.org/10.1364/AO.52.004827

Liu, J. T. C., Jeffries, J. B., \& Hanson, R. K. (2004). Wavelength modulation absorption spectroscopy with $2 \mathrm{f}$ detection using multiplexed diode lasers for rapid temperature measurements in gaseous flows. Applied Physics B: Lasers and Optics, 78(3-4), 503-511. https://doi.org/10.1007/s00340-003-1380-7

Liu, J. T. C., Rieker, G. B., Jeffries, J. B., Gruber, M. R., Carter, C. D., Mathur, T., \& Hanson, R. K. (2005). Near-infrared diode laser absorption diagnostic for temperature and water vapor in a scramjet combustor. Applied Optics, 44(31), 6701. https://doi.org/10.1364/AO.44.006701

Liu, X., Jeffries, J. B., \& Hanson, R. K. (2007a). Measurement of Non-Uniform Temperature Distributions Using Line-of-Sight Absorption Spectroscopy. AIAA Journal, 45(2), 411-419. https://doi.org/10.2514/1.26708

Liu, X., Jeffries, J. B., \& Hanson, R. K. (2007b). Measurements of spectral parameters of watervapour transitions near 1388 and $1345 \mathrm{~nm}$ for accurate simulation of high-pressure absorption spectra. Measurement Science and Technology, 18(5), 1185-1194. https://doi.org/10.1088/0957-0233/18/5/004

Liu, X., Jeffries, J. B., Hanson, R. K., Hinckley, K. M., \& Woodmansee, M. A. (2006). Development of a tunable diode laser sensor for measurements of gas turbine exhaust temperature. Applied Physics B: Lasers and Optics, 82(3), 469-478. https://doi.org/10.1007/s00340-005-2078-9

Mark, G. A. (1998). Diode laser absorption sensors for gas-dynamic and combustion flows. 
Measurement Science and Technology, 9(4), 545. Retrieved from http://stacks.iop.org/0957$0233 / 9 / \mathrm{i}=4 / \mathrm{a}=001$

McDaniel, M. (1983). Flare efficiency study (No. EPA-600/2-83-052). Research Triangle Park, NC: United Stated Environmental Protection Agency. Retrieved from http://nepis.epa.gov/Exe/ZyPURL.cgi?Dockey=P1003QGZ.txt

McEwen, J. D. N., \& Johnson, M. R. (2012). Black Carbon Particulate Matter Emission Factors for Buoyancy Driven Associated Gas Flares. Journal of the Air \& Waste Management Association, 62(3), 307-321. https://doi.org/10.1080/10473289.2011.650040

Migliorini, F., Thomson, K. A., \& Smallwood, G. J. (2011). Investigation of optical properties of aging soot. Applied Physics B: Lasers and Optics, 104(2), 273-283. https://doi.org/10.1007/s00340-011-4396-4

Nelder, J. A., \& Mead, R. (1965). A Simplex Method for Function Minimization. The Computer Journal, 7(4), 308-313. https://doi.org/10.1093/comjnl/7.4.308

O’Neill, P. L., Nicolaides, D., Honnery, D., \& Soria, J. (2004). Autocorrelation Functions and the Determination of Integral Length with Reference to Experimental and Numerical Data. In Australasian Fluid Mechanics Conference.

Pohl, J. H., Tichenor, B. A., Lee, J., \& Payne, R. (1986). Combustion Efficiency of Flares. Combustion Science and Technology, 50(4-6), 217-231. https://doi.org/10.1080/00102208608923934

Poudenx, P., Howell, L. W., Wilson, D. J., \& Kostiuk, L. W. (2004). Downstream Similarity of Thermal Structure in Plumes From Jet Diffusion Flames in a Crossflow. Combustion Science and Technology, 176(3), 409-435. https://doi.org/10.1080/00102200490270139

Rieker, G. B., Jeffries, J. B., \& Hanson, R. K. (2009). Calibration-free wavelength-modulation spectroscopy for measurements of gas temperature and concentration in harsh environments. Appl. Opt., 48(29), 5546-5560. https://doi.org/10.1364/AO.48.005546

Rothman, L. S., Gordon, I. E., Barbe, A., Benner, D. C., Bernath, P. F., Birk, M., ... Champion, J.-P. (2009). The HITRAN 2008 molecular spectroscopic database. Journal of Quantitative Spectroscopy and Radiative Transfer, 110(9-10), 533-572. https://doi.org/10.1016/j.jqsrt.2009.02.013

Saleh, B. E. A., \& Teich, M. C. (2007). Fundamentals of Photonics (2nd ed.). Wiley.

Sand, M., Berntsen, T. K., Von Salzen, K., Flanner, M. G., Langner, J., \& Victor, D. G. (2016). Response of Arctic temperature to changes in emissions of short-lived climate forcers. Nature Climate Change, 6(3), 286-289. https://doi.org/10.1038/nclimate2880

Sanders, S. T., Wang, J., Jeffries, J. B., \& Hanson, R. K. (2001). Diode-laser absorption sensor for line-of-sight gas temperature distributions. Applied Optics, 40(24).

Satterlee, L. (2016). Climate Drones: A New Tool for Oil and Gas Air Emission Monitoring. ENVIRONMENTAL LAW REPORTER, 46(12), 42-58. 
Schiff, H. I., Mackay, G. I., \& Bechara, J. (1994). Air Monitoring by Spectroscopic Techniques. (M. W. Sigrist, Ed.). Concord, Ontario, Canada: Wiley \& Sons, Inc.

Schoonbaert, S. B., Tyner, D. R., \& Johnson, M. R. (2014). Remote ambient methane monitoring using fiber-optically coupled optical sensors. Appl. Phys. B. https://doi.org/10.1007/s00340014-6001-0

Shao, J., Lathdavong, L., Kluczynski, P., Lundqvist, S., \& Axner, O. (2009). Methodology for temperature measurements in water vapor using wavelength-modulation tunable diode laser absorption spectrometry in the telecom C-band. Applied Physics B: Lasers and Optics, 97(3), 727-748. https://doi.org/10.1007/s00340-009-3721-7

Sorensen, C. M. (2001). Light Scattering by Fractal Aggregates: A Review. Aerosol Science and Technology, 35(2), 648-687. https://doi.org/10.1080/02786820117868

Stohl, A., Klimont, Z., Eckhardt, S., Kupiainen, K., Shevchenko, V. P., Kopeikin, V. M., \& Novigatsky, A. N. (2013). Black carbon in the Arctic: the underestimated role of gas flaring and residential combustion emissions. Atmospheric Chemistry and Physics, 13(17), 88338855. https://doi.org/10.5194/acp-13-8833-2013

Strosher, M. T. (2000, October). Characterization of emissions from diffusion flare systems. Journal of the Air \& Waste Management Association.

Thorlabs Inc. (2017). PDA10CS InGaAs Switchable Gain Detector User Guide. Retrieved from https://www.thorlabs.com/thorproduct.cfm?partnumber=PDA20CS

Tkach, R., \& Chraplyvy, A. (1986). Regimes of feedback effects in 1.5-um distributed feedback lasers. Journal of Lightwave Technology, 4(11), 1655-1661. https://doi.org/10.1109/JLT.1986.1074666

Turns, S. R. (2000). An Introduction to Combustion: Concepts and Applications (2nd ed.). USA: McGraw-Hill.

U.S. Environmental Protection Agency. (1999). Nitrogen Oxides (NOx), Why and How They Are Controlled. https://doi.org/EPA 456/F-99-006R

U.S. Environmental Protection Agency. (2012). Report to Congress on Black Carbon (No. EPA450/R-12-001). Research Triangle Park, NC: United States Environmental Protection Agency (U.S. EPA).

Urquhart, P. (1987). Transversely coupled fiber Fabry-Perot resonator: theory. Applied Optics, 26(3), 456-463.

Weyant, C. L., Shepson, P. B., Subramanian, R., Cambaliza, M. O. L., Heimburger, A., Mccabe, D., ... Bond, T. C. (2016). Black carbon emissions from associated natural gas flaring. Environmental Science \& Technology, 50(4), 2075-2081. https://doi.org/10.1021/acs.est.5b04712

Winiger, P., Barrett, T. E., Sheesley, R. J., Huang, L., Sharma, S., Barrie, L. A., ... Gustafsson, Ö. (2019). Source apportionment of circum-Arctic atmospheric black carbon from isotopes and 
modeling. Science Advances, 5(2), eaau8052. https://doi.org/10.1126/sciadv.aau8052

Wood, E. C., Herndon, S. C., Fortner, E. C., Onasch, T. B., Wormhoudt, J., Kolb, C. E., ... Jones, M. (2012). Combustion and Destruction/Removal Efficiencies of In-Use Chemical Flares in the Greater Houston Area. Industrial \& Engineering Chemistry Research, 51(39), 1268512696. https://doi.org/10.1021/ie202717m

World Bank. (2018). New Satellite Data Reveals Progress: Global Gas Flaring Declined in 2017. Retrieved from https://www.worldbank.org/en/news/press-release/2018/07/17/new-satellitedata-reveals-progress-global-gas-flaring-declined-in-2017

Wormhoudt, J., Herndon, S. C., Franklin, J., Wood, E. C., Knighton, B., Evans, S., ... Spellicy, R. (2012). Comparison of remote sensing and extractive sampling measurements of flare combustion efficiency. Industrial \& Engineering Chemistry Research, 51(39), 12621-12629. https://doi.org/10.1021/ie202783m

Wu, K., Li, F., Cheng, X., Yang, Y., Lin, X., \& Xia, Y. (2014). Sensitive detection of CO2 concentration and temperature for hot gases using quantum-cascade laser absorption spectroscopy near $4.2 \mathrm{~lm}$. Applied Physics B: Lasers and Optics, 117(2), 659-666. https://doi.org/10.1007/s00340-014-5880-4

Wu, Q., Wang, F., Li, M., Yan, J., \& Cen, K. (2017). Simultaneous in-situ measurement of soot volume fraction, $\mathrm{H} 2 \mathrm{O}$ concentration, and temperature in an ethylene/air premixed flame using tunable diode laser absorption spectroscopy. Combustion Science and Technology, 189(9), 1571-1590. https://doi.org/10.1080/00102202.2017.1308358

Zhang, G., Liu, J., Xu, Z., He, Y., \& Kan, R. (2016). Characterization of temperature nonuniformity over a premixed CH4-air flame based on line-of-sight TDLAS. Applied Physics B: Lasers and Optics, 122(1), 1-9. https://doi.org/10.1007/s00340-015-6289-4

Zhou, X., Liu, X., Jeffries, J. B., \& Hanson, R. K. (2003). Development of a sensor for temperature and water concentration in combustion gases using a single tunable diode laser. Measurement Science and Technology, 14(8), 1459-1468. https://doi.org/10.1088/0957-0233/14/8/335

Zhu, J., Choi, M. Y., Mulholland, G. W., Manzello, S. L., Gritzo, L. a., \& Suo-Anttila, J. (2002). Measurement of visible and near-IR optical properties of soot produced from laminar flames. Proceedings of the Combustion Institute, 29(2), 2367-2374. https://doi.org/10.1016/S15407489(02)80288-7 


\section{Appendix A Improved Spatial-Resolution LES Simulation Results}

Since the LES datasets inspected in Chapter 2 had a resolution of $2.5 \mathrm{~cm}$ it was possible that the lack of resolution would allow the spectral fit to obtain more accurate estimates of path-averaged $\mathrm{H}_{2} \mathrm{O}$ concentrations. To assess this possibility, another LES dataset was provided by Jeremy Thornock of the University of Utah. This simulation, a single timestep of a methane flame in crossflow had a spatial resolution of $0.5 \mathrm{~cm}$.

A single instance of a spectral fit result is shown in Figure A.1 where the spectral residual value is of similar magnitude $(0.05 \%)$ to the sample fit shown in Figure 3.2. Because of the similarity of the spectral residuals across many spectral fits it was deemed acceptable to use the lower resolution datasets.
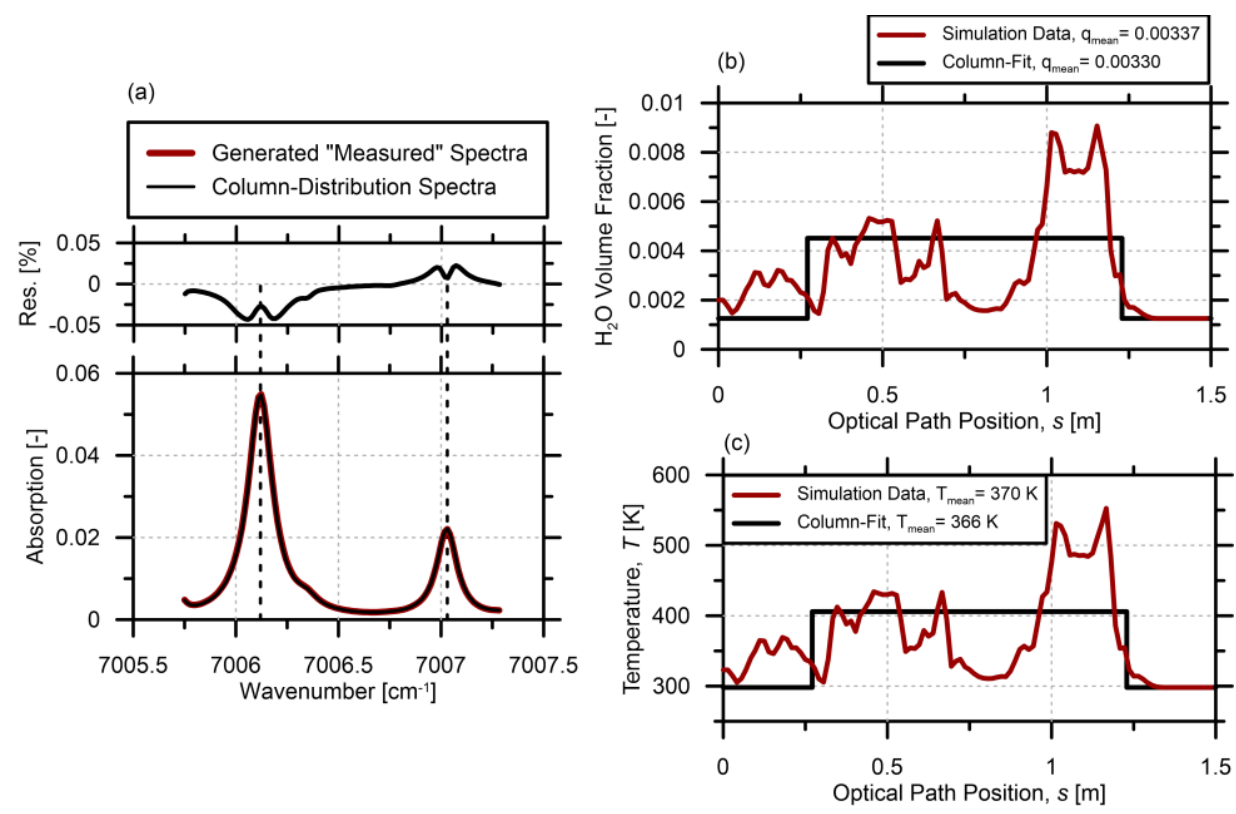

Figure A.1: Sample spectral fitting result from high spatial resolution simulation. Spectral fit (a) shows residuals on the same order as coarser simulation data in Chapter 3; (b) and (c) show comparison of line-of-sight data against effective column fit result. 


\section{Appendix B Time-Resolution of Spectral $\mathrm{H}_{2} \mathrm{O}$ Absorption Signals}

Time-resolution of the path-averaged $\mathrm{H}_{2} \mathrm{O}$ measurement was limited by the $200 \mathrm{~Hz}$ maximum current-tuning sweep rate of the $1428 \mathrm{~nm}$ DFB diode. Since time-resolved soot attenuation measurements indicated that the effective timescales of the measurement were sub-millisecond, it was expected that the spectral absorption due to $\mathrm{H}_{2} \mathrm{O}$ was likely varying throughout the period of the laser sweep. This had the potential to affect the interpretation of the measured $\mathrm{H}_{2} \mathrm{O}$ spectra. At the design and simulation stages of development, in the absence of direct measurement data, it was assumed that the current-tuning sweep rate would be sufficiently high. For this reason, timeresolution of $\mathrm{H}_{2} \mathrm{O}$ spectra was not incorporated into the simulations. With the benefit of experimental results, this issue was revisited to estimate bounds of any impact on analyzed results.

Analysis was performed to test ability of the $\mathrm{H}_{2} \mathrm{O}$ spectra fitting algorithm to correctly interpret acquired signals in which $\mathrm{H}_{2} \mathrm{O}$ volume fraction and temperature may be time-varying. Since the methane flare in crossflow simulations discussed in Chapter 3 did not have sufficient time-resolution for this analysis, synthetic time-varying data were produced using the $500 \mathrm{kHz}$ continuous soot attenuation data from the soot optical property experiments. Path-averaged $\mathrm{H}_{2} \mathrm{O}$ volume fractions were generated with the same variation characteristics as the soot signals. Assuming a fixed column width of $0.75 \mathrm{~m}$ in the synthetic data, the instantaneous path-averaged $\mathrm{H}_{2} \mathrm{O}$ data was used to generate column $\mathrm{H}_{2} \mathrm{O}$ volume fraction data. Along with this time-varying column volume fraction, the column was assumed to be varying in temperature. The temperature was set by assuming a linear relationship between column $\mathrm{H}_{2} \mathrm{O}$ volume fraction and column temperature. This was reasonable since higher column volume fraction implies less air-dilution of 
the combustion products and thus higher temperature. The linear relationship was fixed to produce path-averaged temperatures in the range of 300 to $340 \mathrm{~K}$, which was typical of results in Figure 3.4. Example time-varying synthetic $\mathrm{H}_{2} \mathrm{O}$ signals are shown in Figure B.1.

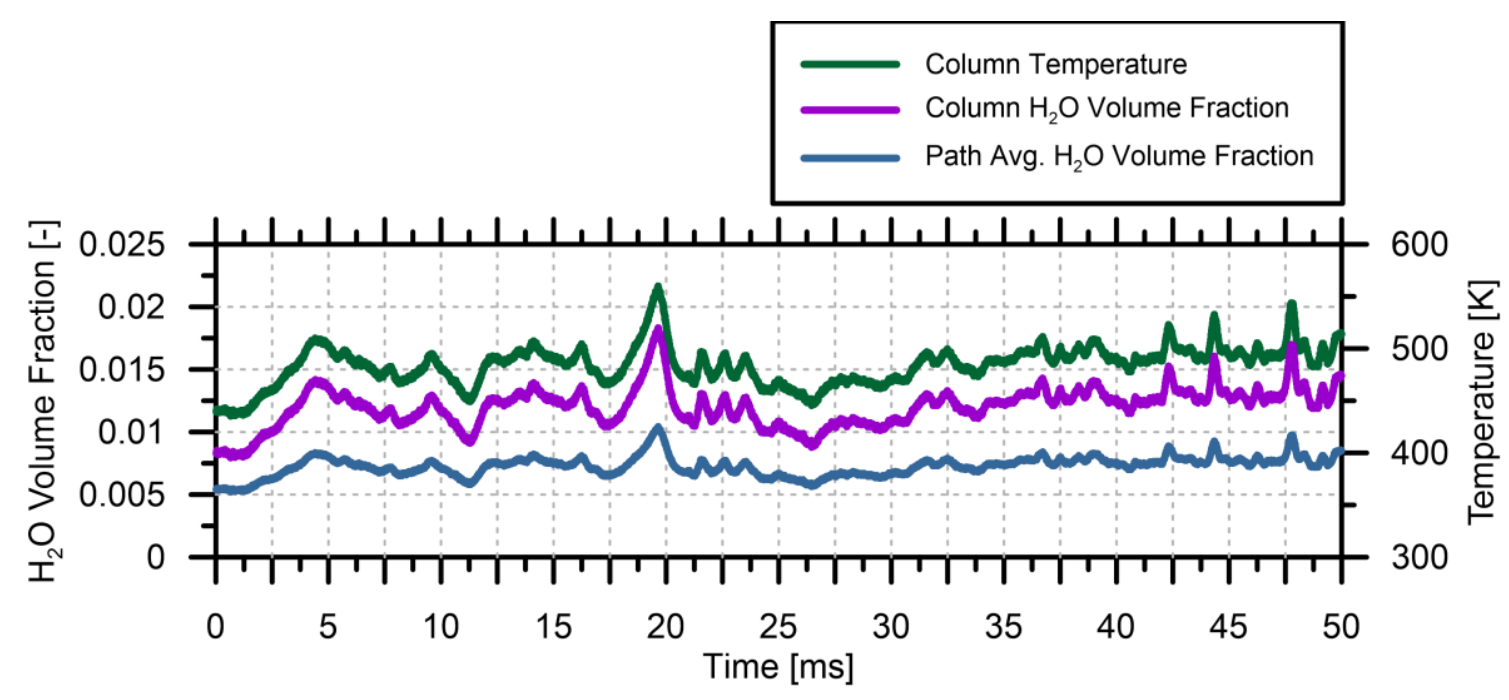

Figure B.1: Varying synthetic $\mathrm{H}_{2} \mathrm{O}$ volume fraction and temperature data generated from measured soot attenuation signals. A 50-ms excerpt of the $1500 \mathrm{~ms}$ synthetic data shown.

The synthetic time-varying data from Figure B.1 was then used in $5 \mathrm{~ms}$ segments to simulate absorption measurements being made in time. At each timestep in the $5 \mathrm{~ms}$ period, the instantaneous column data from Figure B.1 was used to populate an absorption measurement corresponding to the wavenumber position of the laser at that timestep. An example of this is shown in Figure B.2 where the synthetic $\mathrm{H}_{2} \mathrm{O}$ and temperature data populated the lead-lag absorption scans of the two peaks. 


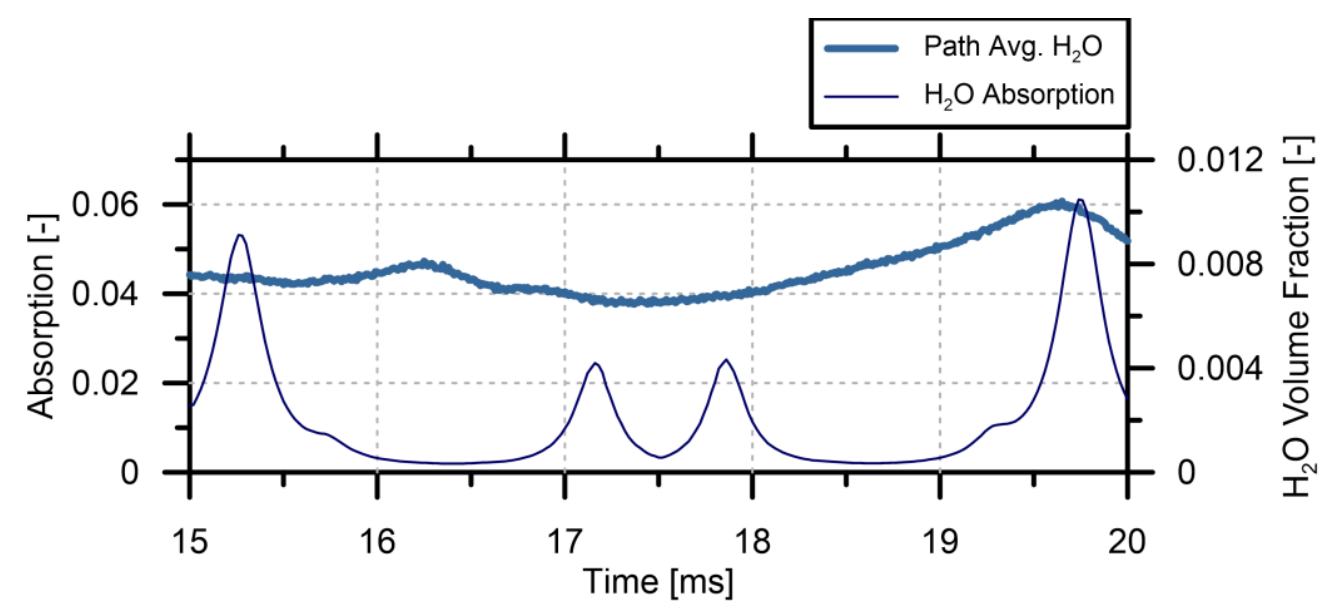

Figure B.2: Time-resolved $\mathrm{H}_{2} \mathrm{O}$ absorption generated from theoretical fluctuations in Figure B.1 for a single 5 ms laser sweep period.

For each current-tuning laser sweep, the lead-lag scans of the $\mathrm{H}_{2} \mathrm{O}$ absorption spectra were aligned by their wavenumber positions and averaged to obtain an effective time-averaged spectrum as shown in Figure B.5, Figure B.4, and Figure B.3. This technique was first described in Section 4.5.1.
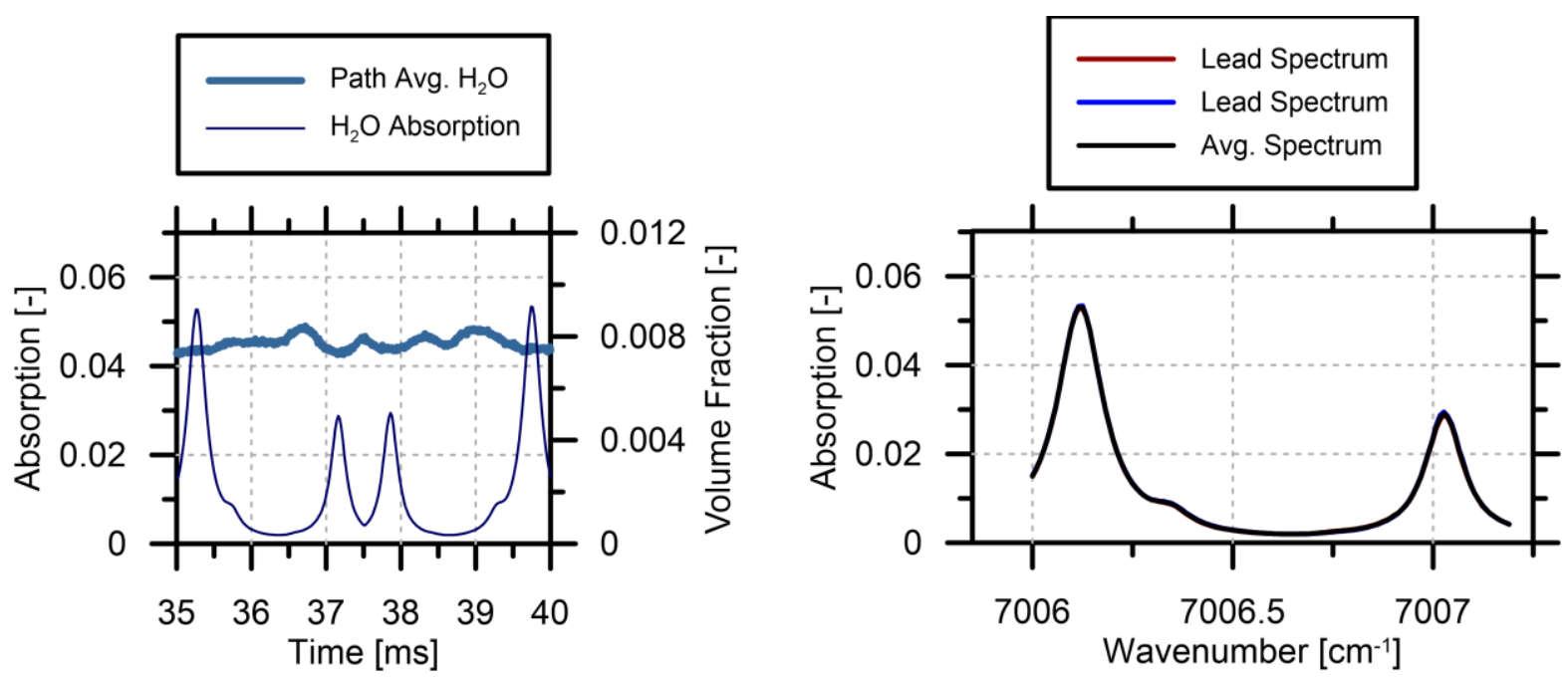

Figure B.3: Sample time-resolved $\mathrm{H}_{2} \mathrm{O}$ spectrum at $35-40 \mathrm{~ms}$ in the synthetic data. Minimal fluctuations of path avg. $\mathrm{H}_{2} \mathrm{O}$ return lead and lag spectra that agree closely. 

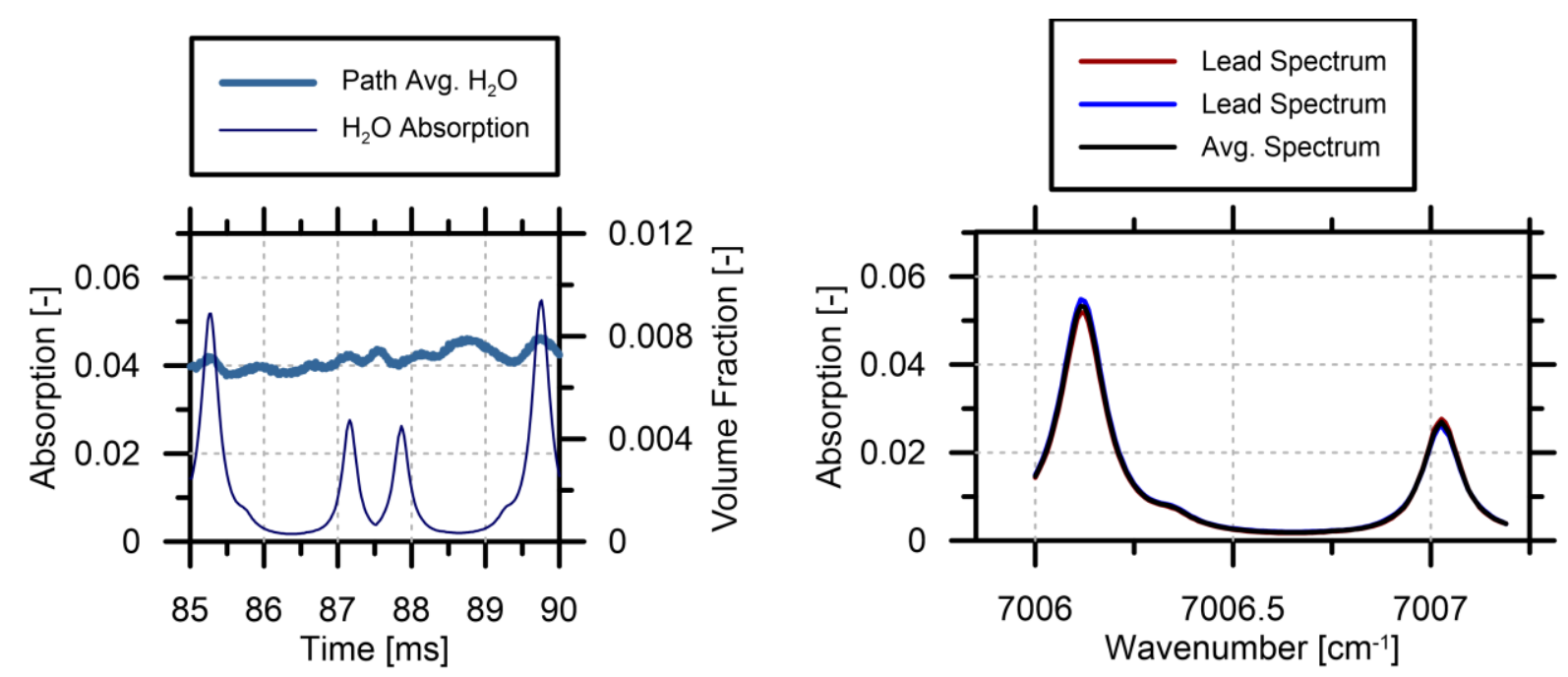

Figure B.4: Sample time-resolved $\mathrm{H}_{2} \mathrm{O}$ spectrum at $85-90 \mathrm{~ms}$ in the synthetic data. Fluctuations of path avg. $\mathrm{H}_{2} \mathrm{O}$ return lead and lag spectra that differ however the average of lead and lag smooths out differences.
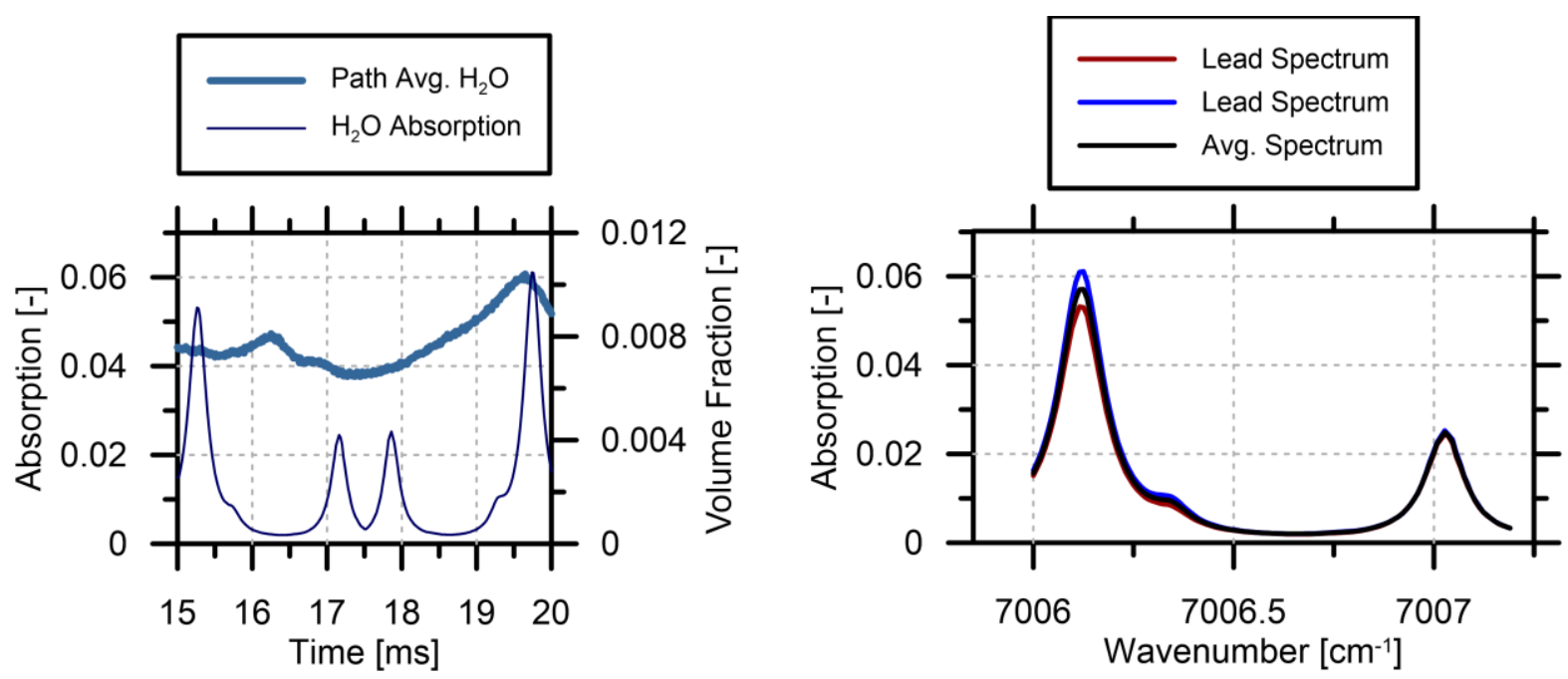

Figure B.5: Sample time-resolved $\mathrm{H}_{2} \mathrm{O}$ spectrum at $15-20 \mathrm{~ms}$ in the synthetic data. Substantial changes in path avg. $\mathrm{H}_{2} \mathrm{O}$ produces noticeably different lead and lag spectra.

The averaged spectrum from each $5 \mathrm{~ms}$ measurement was then fit to a column distribution through a Nelder-Mead optimization scheme. The signal was not corrupted by noise or other confounding factors to observe the effect of averaging the time-varying spectra in isolation. Figure B.6 shows the result of the column distribution fits to the time-averaged spectra shown previously. The example results show relative errors of $0.02 \%, 2.7 \%$, and $7.2 \%$, respectively. 

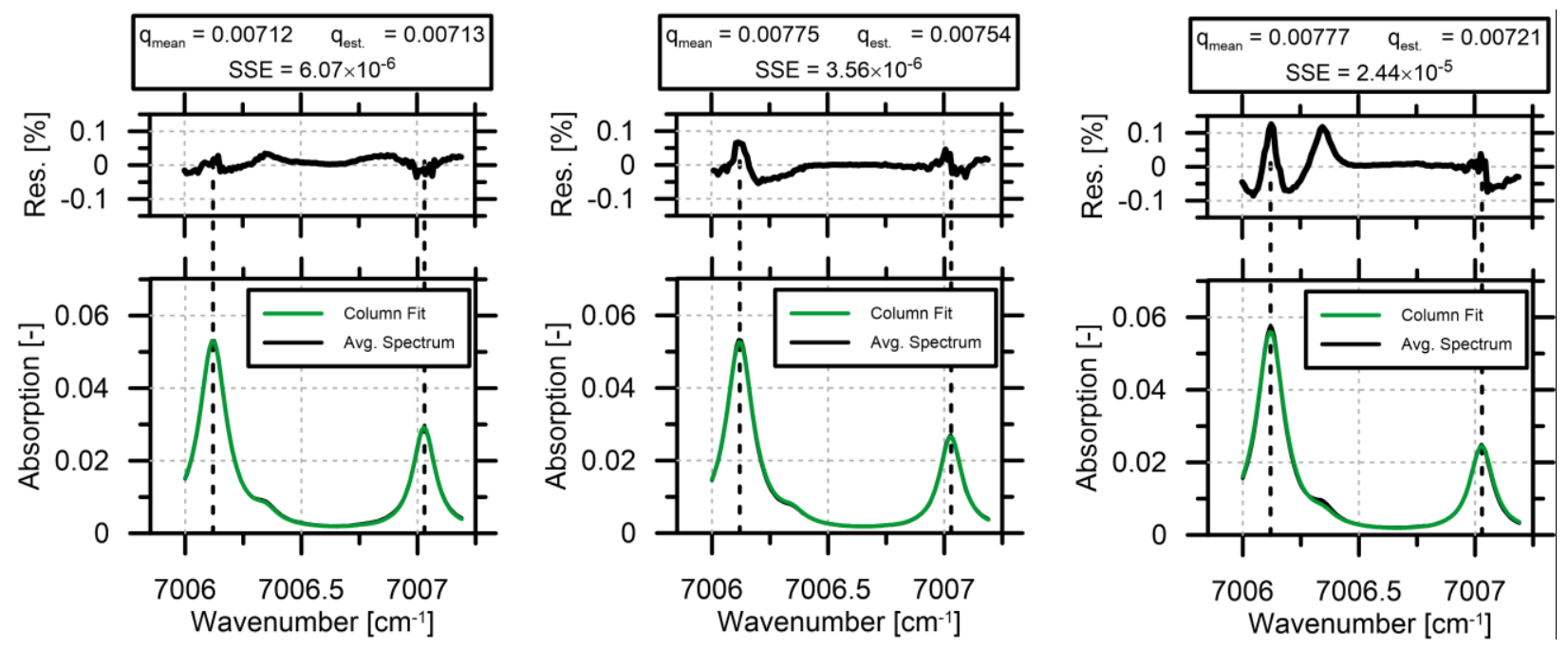

Figure B.6: Column fit results to time-averaged spectra of Figure B.3, Figure B.4, and Figure B.5. Results return relative errors of $0.02 \%, 2.7 \%$, and $7.2 \%$, respectively.

For the 300 results performed for the time-varying spectral scans, the relative error of pathaveraged $\mathrm{H}_{2} \mathrm{O}$ volume fraction had a mean of $0.28 \%$ where $95 \%$ of measurements were within $\pm 5.13 \%$. The result in Figure B.6 showing a relative error of $7.2 \%$ had a probability of occurrence of $\sim 0.4 \%$ assuming a normal distribution of results.

The typical sum-of-squared-errors (SSE) from the spectral fit results of this study were in the range of $10^{-3}$ to $10^{-6}$. This is several orders of magnitude larger than the SSE findings from the simulation results of Chapter 3 which possessed more spatially resolved data than presented here and was corrupted with signal noise. The SSE values from this time-averaging study more closely match values from the flare measurements discussed in Chapter 5, which suggest that the timeaveraging reduces the closeness of fit between measured and theoretical spectral data more than the path-averaging and system noise. For this reason, improved sweep rates and/or more timeresolutions simulations are important recommendations for future work to improve system accuracy. 
If the $\sim 0.28 \%$ average error $( \pm 5.13 \%$ with $95 \%$ confidence) from time-averaging in this Appendix is assumed to be independent of the $-0.25 \%$ average error $( \pm 6.74 \%, 95 \%$ confidence $)$ in fitting spectra to compute the path-averaged volume fraction discussed in Chapter 3 , the overall relative error of path-averaged estimates of $\mathrm{H}_{2} \mathrm{O}$ would have a standard deviation of $4.3 \%$ roughly centered about $0.06 \%$. Importantly, as discussed in Section 6.1, finer time-resolution in the present experiment would most likely indicate even broader variations in soot- $\mathrm{H}_{2} \mathrm{O}$ correlation that might currently be obscured by time-averaging. 


\section{Appendix C Spectral Fitting Results of Flare Experiments}

Not all experimental conditions from the original test matrix were accepted and used in further analyses. Flares burning methane and ethylene were rejected for producing plume conditions that were beyond the capability of the optical system to measure. This section briefly presents results from methane and ethylene flares and illustrates the basis for which these tests were rejected.

Figure C.1 plots a histogram of soot volume fraction (SVF) measurements made of the methane flare and compares them to previously shown results from the hydrogen flare (in which no soot is present). Visually, and from the noted statistics, it is not possible to distinguish the presence of soot in the methane flare above the base light attenuation caused by light loss due to beam steering and measurement noise. For this reason, all methane flare results were rejected due to insufficient soot signal.

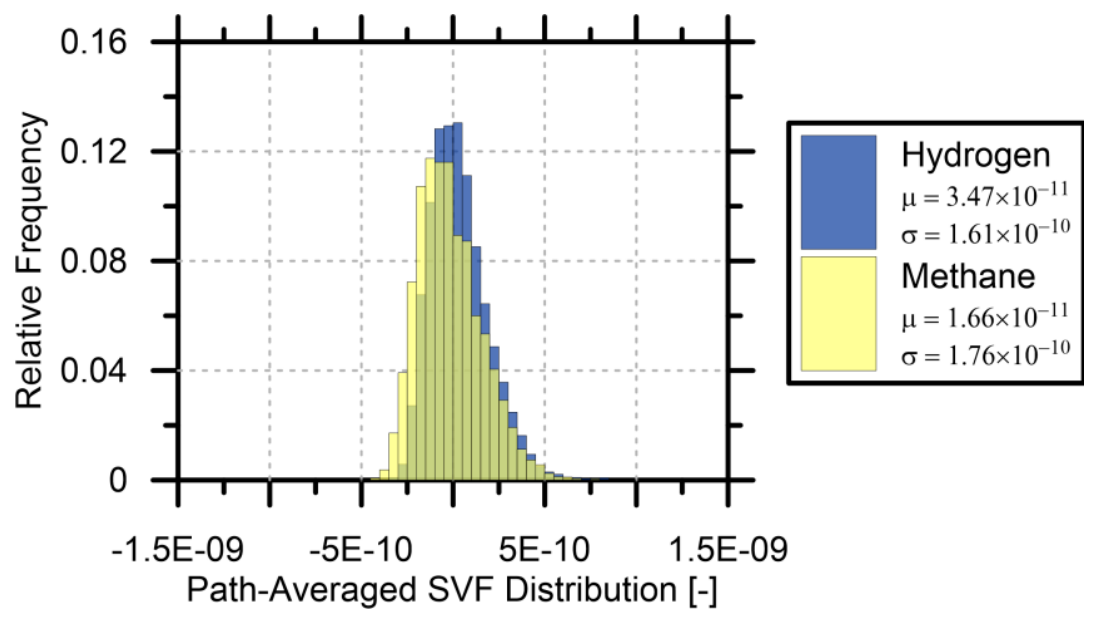

Figure C.1: Comparison of inferred soot volume fraction in methane and hydrogen flares. No significant increase in SVF is apparent in the methane flare.

By contrast, the ethylene flare produced large amounts of soot, causing light attenuation often in excess of $10 \%$. Although the soot presence was easily detectable, uncertainty of the soot optical 
properties began to obscure or distort the $\mathrm{H}_{2} \mathrm{O}$ absorption spectra when attempting to isolate these spectra. Figure C.2 shows a sample $\mathrm{H}_{2} \mathrm{O}$ and soot signal from the ethylene flare tests.

(a)

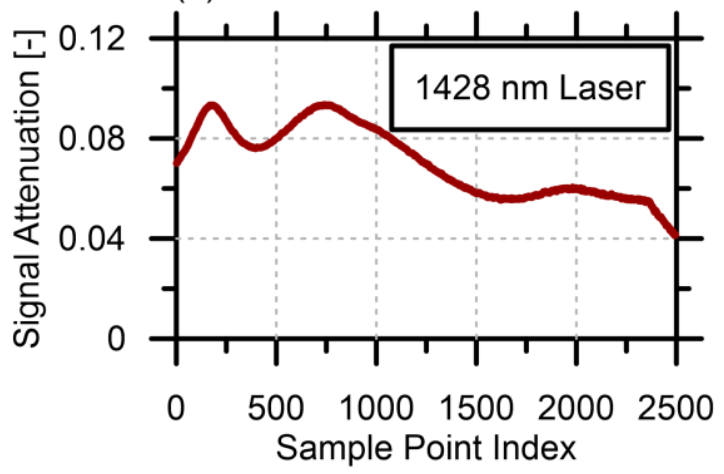

(b)

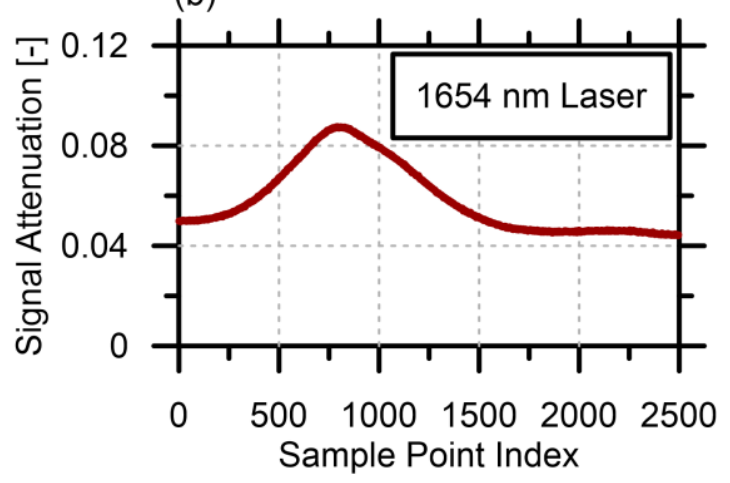

Figure C.2: Sample attenuation signal on (a) $1428 \mathrm{~nm}\left(\mathrm{H}_{2} \mathrm{O}\right)$ and (b) $1654 \mathrm{~nm}$ (soot) lasers. A large light-attenuating soot structure is observed which peaks near the 800 index.

When the $\mathrm{H}_{2} \mathrm{O}$ spectra were isolated from the soot attenuation on the $1428 \mathrm{~nm}$ laser, uncertainty of the soot extinction coefficient ratio (ECR) between wavelengths combined with high soot presence (and thus high light attenuation) produced isolated spectra where residual soot attenuation is observed. Figure C.3 shows the isolated $\mathrm{H}_{2} \mathrm{O}$ spectra estimated for the sample measurements of Figure C.2. At $7006.5 \mathrm{~cm}^{-1}$, there is an apparent negative absorption which is physically impossible and implies that the measured soot ECR was underestimated. Although in this example the main residual lies in between the absorption peaks, the presence of this residual suggests the ECR may bias all results low if soot attenuation is sufficiently high.

The apparent underestimation of the extinction coefficient ratio causes biasing of the isolated $\mathrm{H}_{2} \mathrm{O}$ spectra for ethylene flares where attenuation often exceeds $10 \%$. For the flares tests discussed in Chapter 5, typical light attenuation due to soot did not typically exceed 3\% meaning any biasing during the $\mathrm{H}_{2} \mathrm{O}$ isolation will be reduced by at least a factor of three. It is difficult to quantify the apparent error in the ECR measurement because the example in Figure C.3 may have also been subjected to a beam steering event that would contribute to the distortion of $\mathrm{H}_{2} \mathrm{O}$ spectra. 
In any case, since this result was not the only result with distorted spectra from the ethylene tests, these experiments were deemed unreliable and were not considered in subsequent analyses.

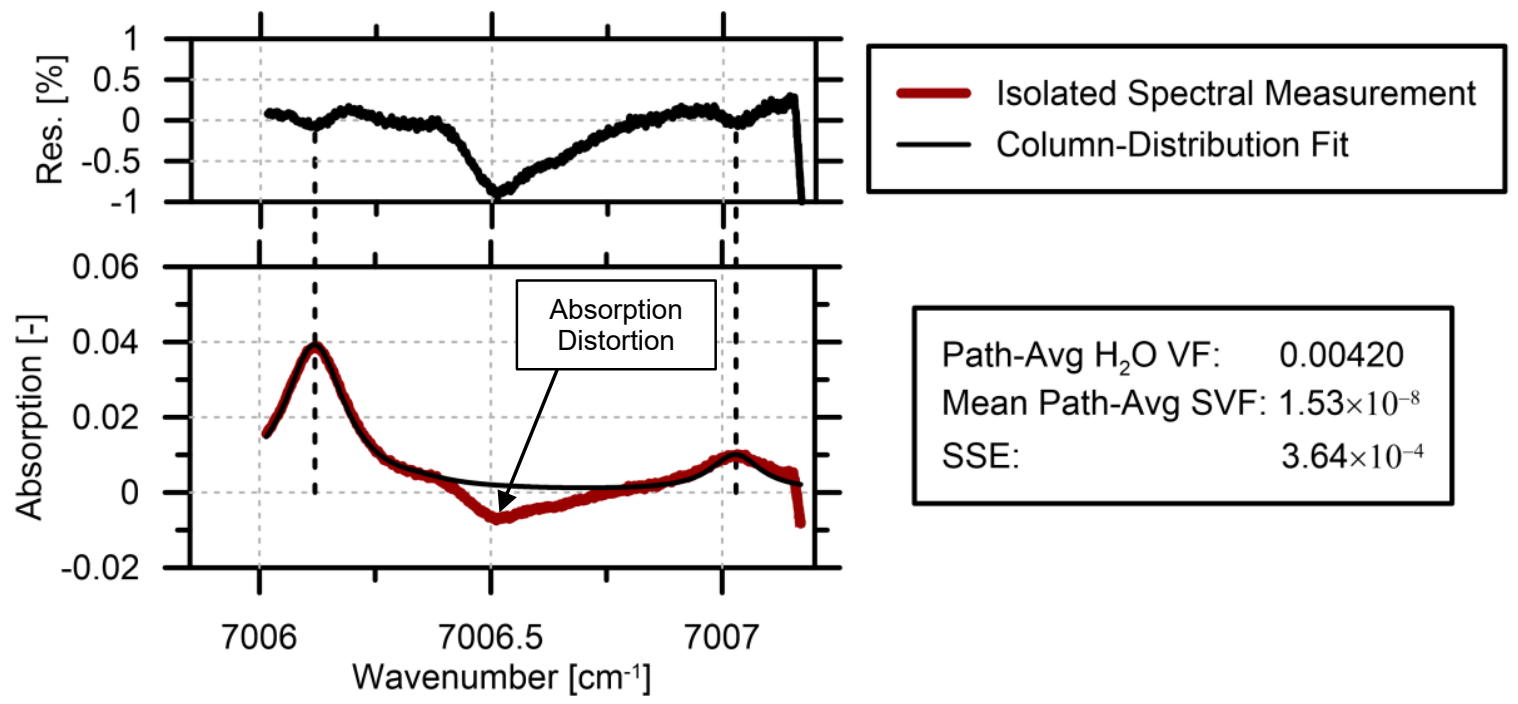

Figure C.3: Uncertainties in soot optical properties produces significant distortion of the isolated $\mathrm{H}_{2} \mathrm{O}$ absorption peaks. 


\section{Appendix D Data Filtering and Inherent Measurement Skew}

Not all measurements from the flare tests using the Bakken and Ecuador mixtures were used in the

analysis in Chapter 5. Results were filtered based on the closeness of fit between the measured $\mathrm{H}_{2} \mathrm{O}$ spectra and the theoretical spectra produced by the column distribution fit. This was done to eliminate results that for various reasons may have produced high errors in $\mathrm{H}_{2} \mathrm{O}$ estimates stemming from poor spectral fitting of measured to theoretical spectra. Spatial and temporal averaging that resulted in spectra not well-described by an instantaneous column distribution spectrum was likely the main contributor, however system noise and light loss due to beam steering would also affect closeness of spectral fitting. This appendix verifies that the chosen SSE limit would not adversely affect interpreted results.

The sum-of-squared-errors (SSE) of the column distribution fit was used as the metric by which the data was filtered. The specific cut-off SSE was selected to be $10^{-4}$ [-]. This value was roughly the largest SSE value measured during the $\mathrm{H}_{2} \mathrm{O}$ vapour flow-through cell experiments discussed in Section 4.6. This value represents a closeness of fit that is not significantly affected by beam steering or temporal and spatial averaging. Figure D.1 shows a sample fit from the flowthrough experiments that represents an SSE of $1.08 \times 10^{-4}$. 


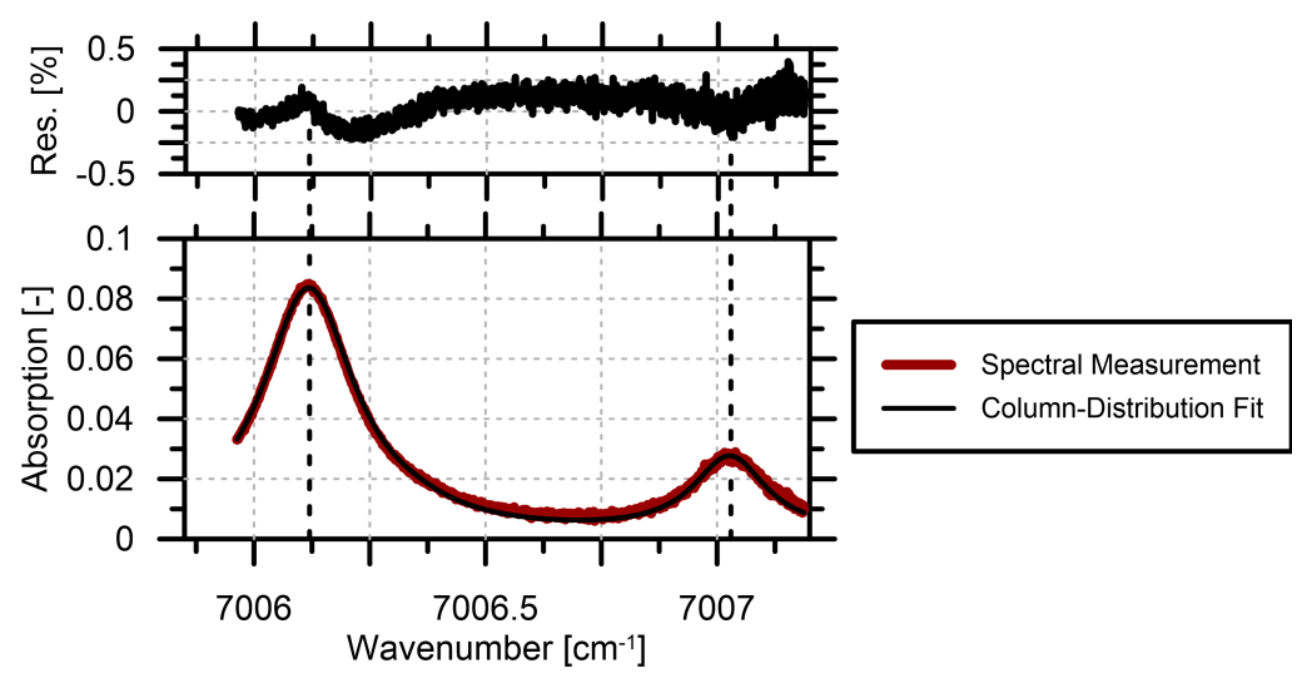

Figure D.1: Sample spectral measurement from flow-through $\mathrm{H}_{2} \mathrm{O}$ vapour experiments conducted in Chapter 4. This sample represents an SSE of $1.08 \times 10^{-4}$, the maximum SSE accepted.

While it is possible that the SSE cut-off of $10^{-4}[-]$ is too restrictive and should be relaxed, Figure D.2 suggests that using less stringent SSE cut-off values would only increase the apparent skewness of the soot- $\mathrm{H}_{2} \mathrm{O}$ ratio. The figure shows the skewness of the soot- $\mathrm{H}_{2} \mathrm{O}$ results after using varying cut-off values for both the Bakken and Ecuador test results. If the $10^{-4}[-]$ cut-off were relaxed, the resulting soot- $\mathrm{H}_{2} \mathrm{O}$ skewness would tend to increase which means that using this cutoff will produce a conservative estimate of the skewness. The $10^{-4}[-]$ cut-off is a reasonable value based on the limitations of spectral fitting on measurements performed in a controlled experiment. A more restrictive value would likely filter out most results and does not appear to substantially affect skewness in Figure D.2. 

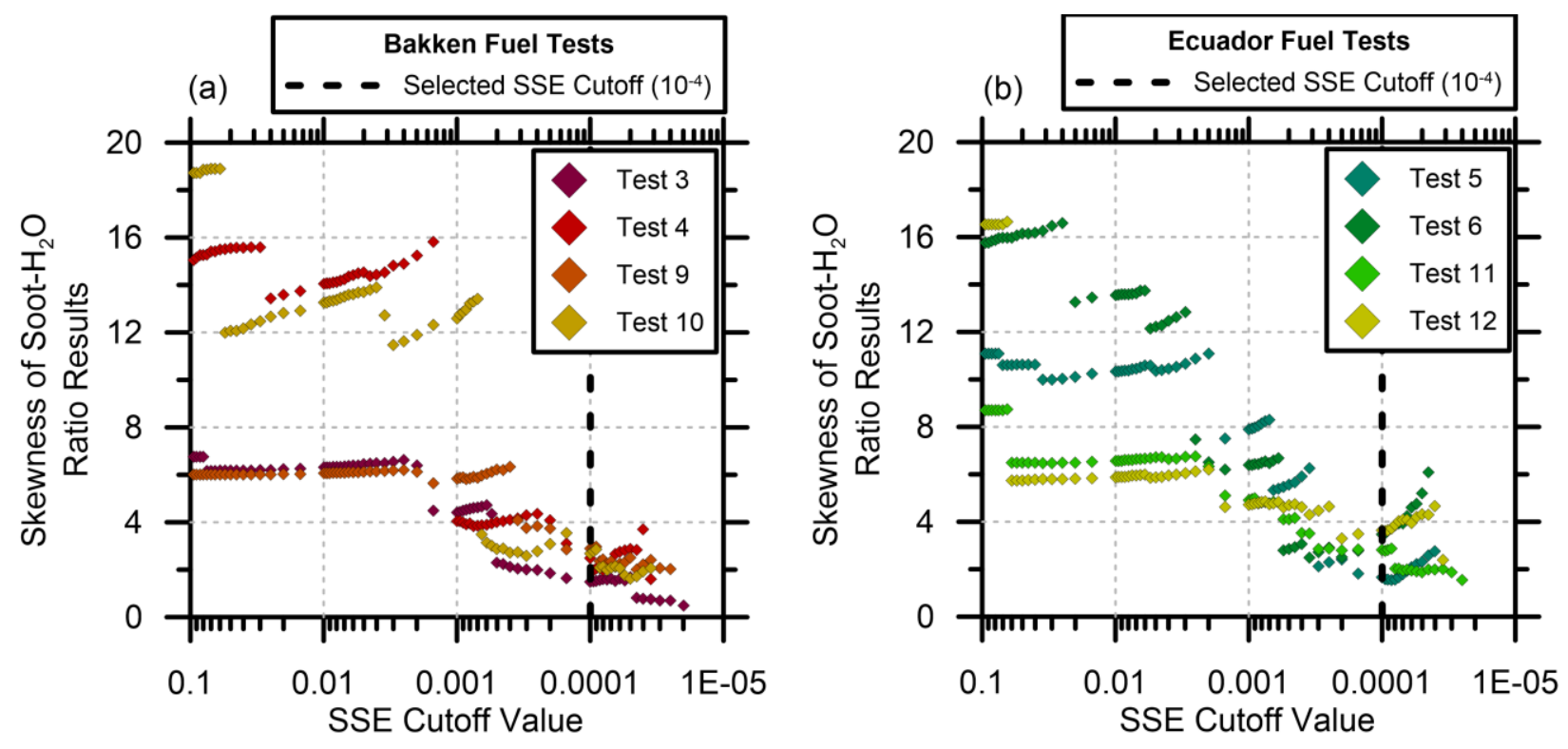

Figure D.2: Affect of changing the SSE cut-off value on the skewness of the Soot- $\mathrm{H}_{2} \mathrm{O}$ ratio results.

The skewness of the soot- $\mathrm{H}_{2} \mathrm{O}$ ratio distributions from Figure D.2 at the cut-off SSE indicate skewness values between 1.63 and 3.48. These conservative estimates of the skewness of the soot$\mathrm{H}_{2} \mathrm{O}$ distributions must be compared with the inherent tendency of a ratio of two uncertain nonnegative numbers to produce a skewed distribution. Typical values of soot and $\mathrm{H}_{2} \mathrm{O}$ volume fraction were assumed along with estimated measurement errors expanded upon in previous analyses. Mean values of soot volume fraction and combustion-derived $\mathrm{H}_{2} \mathrm{O}$ volume fraction were assumed to be $3.64 \times 10^{-9}$ and 0.00261 , respectively. These values were found in Test 12 of an Ecuador fuel mixture at 60 SLPM on the $76.2-\mathrm{mm}$ diameter burner. The error on the soot measurement is driven by system noise and light loss due to beam steering with a standard deviation of $1.61 \times 10^{-10}$ (Section 4.9). The relative error of $\mathrm{H}_{2} \mathrm{O}$ estimates was reported as a mean of $-0.25 \%$ ( $\pm 6.74 \%$ for $95 \%$ of measurements) for spatial averaging and system noise (from Section 3.2$)$, and a mean of $0.28 \%( \pm 5.13 \%$ for $95 \%$ of measurements $)$ for temporal averaging (from Appendix B). Although these sources of error were treated separately, the effective combined error of $\mathrm{H}_{2} \mathrm{O}$ estimates had a mean relative error of $0.06 \%$ with $95 \%$ of measurements 
being within $\pm 8.49 \%$. The relative error results were quoted on the path-averaged $\mathrm{H}_{2} \mathrm{O}$ volume fraction whose mean value was 0.00443 .

Assuming normal distributions of each error source, a 50,000-iteration Monte Carlo simulation was used to determine the inherent skewness produced by the ratio of uncertain soot and $\mathrm{H}_{2} \mathrm{O}$ estimates from the optical measurement system. The result of the simulation is shown in Figure D.3. The distribution of soot $-\mathrm{H}_{2} \mathrm{O}$ ratio had a standard deviation of $1.09 \times 10^{-7}$ and a skewness of 0.37 .

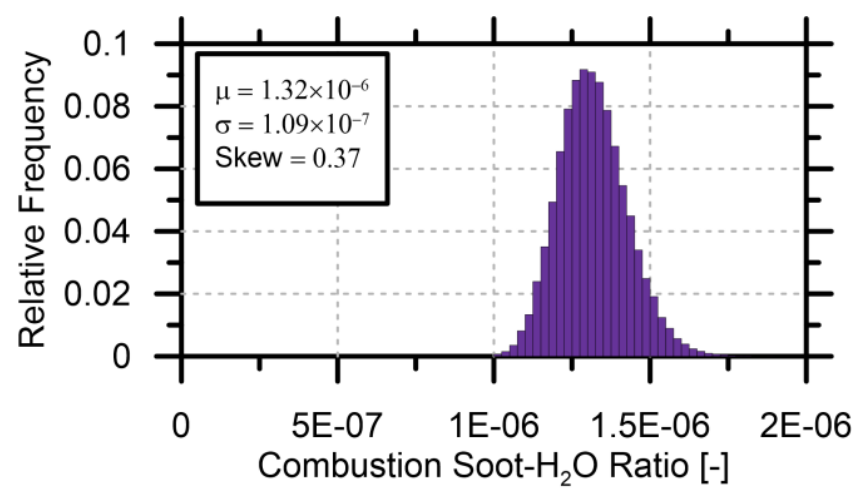

Figure D.3: Distribution of Soot- $\mathrm{H}_{2} \mathrm{O}$ ratio assuming normal distribution of parameters with errors estimated in previous work in this thesis. Inherent skewness of 0.37 was observed for the typical values of the two species.

The skewness of the distribution of uncertain parameters is 0.37 . If errors were accurately quantified from previous work in this thesis, any skewness in the distribution of the soot- $\mathrm{H}_{2} \mathrm{O}$ ratio above 0.37 is a true skewness inherent in the path-averaged soot- $\mathrm{H}_{2} \mathrm{O}$ ratio. In Chapter 5 , all results of the Bakken and Ecuador fuel mixtures tests produced skewness of between 1.62 and 3.48 which implies that there exists skewness in the distribution of soot- $\mathrm{H}_{2} \mathrm{O}$ ratios on the path-average beyond this inherent minimum. 